Universidade de Brasília

Instituto de Ciências Exatas

Departamento de Matemática

\title{
Relação de alcance em dígrafos transitivos e a propriedade $\mathrm{Z}$
}

\author{
por \\ Regiane Lopes de Oliveira
}

Brasília 
Ficha catalográfica elaborada automaticamente, com os dados fornecidos pelo(a) autor(a)

Relação de alcance em dígrafos transitivos e a propriedade Z / Regiane Lopes de Oliveira;

orientador Daniela Amorim Amato. -- Brasília, 2016. $70 \mathrm{p}$.

Dissertação (Mestrado - Mestrado em Matemática) -Universidade de Brasília, 2016.

1. Dígrafos Transitivos. 2. Relações de alcance. 3. Propriedade Z. 4. Grupos Nilpotentes. I. Amato, Daniela Amorim, orient. II. Título. 
Universidade de Brasília

Instituto de Ciências Exatas

Departamento de Matemática

\title{
Relação de alcance em dígrafos transitivos e a
}

$$
\text { propriedade } Z
$$

\author{
por
}

REGIANE LOPES DE OLIVEIRA *

Dissertação apresentada ao Departamento de Matemática da Universidade

de Brasília, como parte dos requisitos para obtenção do grau de

\section{MESTRE EM MATEMÁTICA}

Brasília, 09 de março de 2016.

Comissão Examinadora:

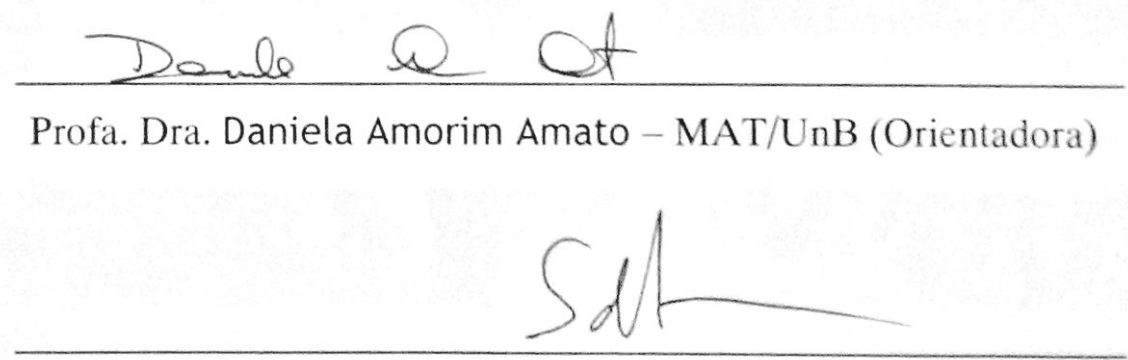

Prof. Dr. Csaba Schneider - UFMG (Membro)

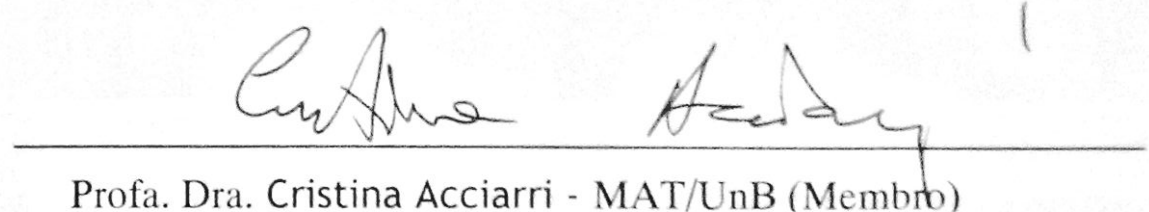

* O autor foi bolsista do CNPq durante a elaboração desta dissertação. 
À minha mãe Cleunice e às minhas irmãs Raquel e Rute 


\section{Agradecimentos}

Primeiramente, agradeço a Deus por todas as bençãos alcançadas.

Aos meus familiares, minha mãe, minhas irmãs e meu pai. Em especial minha mãe pelo carinho, pelo amor e por me ajudar a nunca desistir dos meus sonhos.

À professora Daniela Amato meus sinceros agradecimentos pela orientação, por toda a dedicação, paciência, disposição, pela confiança depositada em mim e por me fazer encantar com a Teoria dos Grafos. Muito obrigada por tudo.

Aos professores da banca examinadora, Cristina Acciarri e Csaba Schneider.

Aos professores do Departamento de matemática da UnB pelos conhecimentos matemáticos transmitidos. Em especial às professoras Cátia Regina, Cristina Acciarri e Daniela Amato, por me fazerem redescobrir o quanto é maravilhoso estudar matemática.

A todos os professores que me ajudaram a chegar até aqui. Especialmente os do departamento de matemática da UFMT/CUR.

À Sunamita, não há palavras para descrever e nem números que possam expressar o quanto eu sou grata pela sua amizade e pelo seu apoio dedicado a mim.

A todos os meus amigos, em especial, Ana, Bruno, Christe, Gláucia, Karen, Karol, Keidna, Lumena, Maria, Mayra, Nathália, Rafael, Sara, Welber e Welinton. Agradeço a vocês pela amizade, pelo apoio, e claro, pelos momentos felizes repletos de muitas risadas.

Às minhas amigas de moradia Aline e Luciana, pela convivência fantástica e pela amizade.

Ao $C N P Q$ pelo apoio financeiro a este trabalho.

Enfim, agradeço a todos que de certa forma me ajudaram a chegar até aqui. 


\section{Resumo}

Neste trabalho estudamos uma família de relações de equivalência definidas nos vértices de um dígrafo, as chamadas relações de alcance. Primeiramente, apresentamos diversas propriedades gerais das relações em questão e, então estudamos tais relações em conexão com propriedades de grupos de automorfismos de dígrafos transitivos. Em particular, o principal resultado apresentado mostra que se um dígrafo transitivo $D$ admite um subgrupo nilpotente $H$ do grupo de automorfismo $\operatorname{Aut}(D)$ de $D$, agindo com uma quantidade finita de órbitas sobre $D$, então a classe de nilpotência de $H$ e o número de órbitas estão intimamente relacionadas com determinadas propriedades das relações de alcance.

Além disso, estudamos como as propriedades das relações de alcance estão relacionadas com outras propriedades do dígrafo, tais como 'ter a propriedade Z' e condições de crescimento.

Palavras-Chave: Dígrafos transitivos; Relações de alcance; Propriedade Z; Grupos Nilpotentes. 


\section{Abstract}

In this work we study a family of equivalence relations defined in the vertices of a digraph, the called reachability relations. First, we present several general properties of the relations in question and then we study such relations in connection with properties of automorphism groups of transitive digraphs. In particular, the main result shows that if a transitive digraph $D$ admits a nilpotent subgroup $H$ of the automorphism group $A u t(D)$ of $D$ acting with finitely many orbits on $D$, then the nilpotent class of $H$ and the number of orbits are closely related to determined properties of the reachability relations.

Futhermore, we study how properties of the reachability relation are related with others properties of digraphs, such as 'having propety Z' and growth conditions.

Key-Words: Transitive digraphs; Reachability relations; Property Z; Nilpotent groups. 


\section{Sumário}

1 Preliminares 1

2 Dígrafos e Grupos $\quad 7$

2.1 Definições . . . . . . . . . . . . . . . . . . . . . . . . . 7

2.2 Propriedade Z . . . . . . . . . . . . . . . . . . . . . . 11

2.3 Dígrafos de Cayley . . . . . . . . . . . . . . . . . . . . . . . . 12

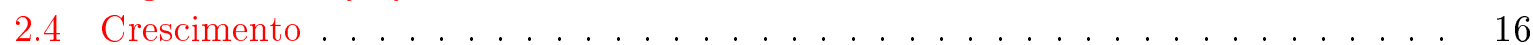

3 Relações de alcance em dígrafos $\quad 19$

3.1 Algumas propriedades de $R_{k}^{+}$e $R_{k}^{-} \ldots \ldots \ldots \ldots \ldots \ldots$. . . . . . . . 21

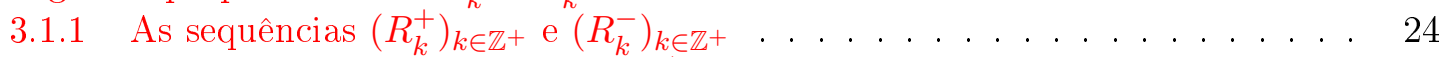

3.1.2 As classes de equivalências de $R_{k}^{+}$e $R_{k}^{-} \ldots \ldots \ldots \ldots \ldots \ldots$

3.2 Propriedade $\mathrm{Z}$ e crescimento . . . . . . . . . . . . . . . 36

4 Relações de alcance e grupos com crescimento polinomial 41

4.1 Resultados auxiliares . . . . . . . . . . . . . . . . . . . . . . 42

4.2 As relações $R^{+}$e $R^{-}$em dígrafos transitivos . . . . . . . . . . . . . 47

4.3 Observações finais . . . . . . . . . . . . . . . . . . . 57

$\begin{array}{ll}\text { Referências bibliográficas } & 60\end{array}$ 


\section{Introdução}

Um dígrafo $D$ é um par $(V D, E D)$, onde $V D$ é um conjunto não vazio e $E D \subseteq V D \times V D$ é um conjunto de pares ordenados de elementos de $V D$. Os elementos de $V D$ são denominados vértices e os elementos de $E D$ de arestas. Um passeio $W=\left(v_{0}, \epsilon_{1}, v_{1}, \epsilon_{2}, \ldots, \epsilon_{n}, v_{n}\right)$ de $v_{0}$ até $v_{n}$ é uma sequência de $n+1$ vértices (não necessariamente pares distintos) $v_{0}, v_{1}, \ldots, v_{n}$ e $n$ indicadores $\epsilon_{1}, \ldots, \epsilon_{n} \in\{-1,1\}$, tal que para todo $j \in\{1,2, \ldots, n\}$ temos:

$$
\begin{gathered}
\epsilon_{j}=1 \Rightarrow\left(v_{j-1}, v_{j}\right) \in E W \\
\epsilon_{j}=-1 \Rightarrow\left(v_{j}, v_{j-1}\right) \in E W
\end{gathered}
$$

Um passeio é dito alternado se os indicadores $\epsilon_{i}$ alternam.

Em [5] foi introduzida a noção de relação de alcance definida no conjunto de arestas de um dígrafo, denotada por $\mathcal{A}$. Dizemos que uma aresta $e^{\prime}$ é alcançada por uma aresta $e$, se existe um passeio alternado cuja a primeira aresta é e e a última aresta é $e^{\prime}$.

Em [5] os autores tentaram classificar dígrafos altamente transitivos usando propriedades da relação $\mathcal{A}$, com isto foram proposto um número interessante de problemas. Alguns destes problemas foram estudados, em [10] por Malnič, Marušič, Seifter e Zgrablič e como fruto deste estudo surgiu o interesse de estudar uma família de relações de alcance agora sendo definida nos vértices de um dígrafo. Assim, juntamente com $\breve{S}$ parl, definiram em [11] relações de alcance nos vértices.

Uma definição informal (para uma definição precisa veja seção 3) é a seguinte. Primeiro, o peso de um passeio $W$ é definido como a diferença entre o número de arestas percorridas no mesmo sentido de $W$, e o número de arestas percorridas no sentido contrário a $W$. Seja $k \geq 1$ um inteiro. Então um vértice $u$ está $R_{k}^{+}$relacionado com um vértice $v$, se existe um passeio $W$ de peso 0 de $u$ a $v$ tal que todo subpasseio de $W$ com vértice inicial $u$ tem peso variando no intervalo $[0, k]$. De forma similar, dizemos que um vértice $u$ está $R_{k}^{-}$relacionado com um vértice $v$, se existe um passeio $W$ de peso 0 ligando $u$ a $v$ tal que todo subpasseio de $W$ com vértice inicial $u$ tem peso no intervalo $[-k, 0]$. Observe que $R_{k}^{+}$e $R_{k}^{-}$são relações de equivalências se $D$ é um dígrafo com grau de entrada e grau de saída pelo menos igual a 1 . E se $R_{k}^{+}=R_{k+1}^{+}$ 
para algum $k$ então definimos o expoente $\exp ^{+}(D)$ de um dígrafo $D$, como o menor inteiro tal que $R_{k}^{+}=R_{k+1}^{+}$. Agora se $R_{k}^{+} \neq R_{k+1}^{+}$, para todo inteiro $k$, então $\exp ^{+}(D)=\infty$. Similarmente definimos o expoente $\exp ^{-}(D)$.

Também em [5] foi proposto o seguinte problema:

Problema 1. Existe um dígrafo conexo, altamente arco transitivo e localmente finito tal que a relação de alcance nas arestas é universal?

Este problema ficou um bom tempo em aberto, sendo finalmente solucionado em [6]. Veja que o Problema 1 também é solucionado, usando relações de alcance nos vértices, se supormos apenas que o dígrafo é conexo e tem um laço em cada vértice, ou se o dígrafo é conexo, tendo um ciclo direcionado de comprimento 2 em cada vértice e contém um passeio fechado de comprimento ímpar (veja Proposição 3.6).

Temos também que propriedades de $R_{k}^{+}$e $R_{k}^{-}$estão intimamente relacionadas com propriedade de dígrafos, tal como condição para um dígrafo ter crescimento exponencial como pode ser visto no teorema a seguir.

Teorema 3.21. Seja D um digrafo conexo, transitivo e localmente finito. Se pelo menos um dos expoentes $\exp ^{+}(D)$ e $\exp ^{-}(D)$ é infinito então D tem crescimento exponencial.

Com isto temos que um grupo finitamente gerado tem crescimento exponencial se para um dos dígrafos de Cayley $D$ de $G$, pelo menos um dos expoentes $\exp ^{+}(D)$ e $\exp ^{-}(D)$ é infinito.

Dessa forma, se $G$ não tem crescimento exponencial, ambos os expoentes $\exp ^{+}(D)$ e $\exp ^{-}(D)$ são finitos. Neste caso, surge a seguinte pergunta:

Pergunta. O que pode ser dito sobre propriedades das relaçôes de alcance em dígrafos de Cayley de um grupo finitamente gerado com crescimento polinomial?

Por um resultado de Gromov, em [8], temos que um grupo finitamente gerado tem crescimento polinomial se, e somente se, ele contém um subgrupo normal nilpotente com índice finito. Assim, a resposta para a pergunta acima é dada pelo seguinte resultado:

Teorema 4.15. Seja $G$ um grupo agindo transitivamente sobre um digrafo conexo $D$ e seja $N \unlhd G$ normal nilpotente de classe $r$ agindo com $m$ órbitas sobre $D$, onde $1 \leq m<\infty$. Então $\exp ^{+}(D)=\exp ^{-}(D) \leq m(r+1)-1$.

Temos também que propriedades de $R_{k}^{+}$e $R_{k}^{-}$estão intimamente relacionadas com propriedades de dígrafos, tal como condição para ter propriedade $Z$ (veja Proposições 3.20 e 3.19). Um dígrafo $D$ tem propriedade $Z$ se existe um homomorfismo sobrejetor $\phi: D \rightarrow Z$, onde $Z$ é o 
dígrafo que tem como conjunto de vértices $\mathbb{Z}$ e conjunto de arestas $\{(i, i+1) \mid i \in \mathbb{Z}\}$.

Diante disso, concluímos que o presente trabalho tem como objetivo principal estudar as relações de alcance definidas nos vértices de um dígrafo, tais como suas propriedades e suas conexões com propriedades de grupo de automorfismo de dígrafos transitivos e propriedade $Z$. Tal estudo será realizado através de resultados demonstrados em [11] e [13] e está dividido em quatro capítulos. No capítulo 1 relembraremos alguns conceitos básicos da teoria de grupos utilizados neste trabalho. No capítulo 2 daremos algumas definições básicas da teoria de dígrafos. No capítulo 3 estudaremos propriedades de $R_{k}^{+}$e $R_{k}^{-}$e suas conexões com propriedades de dígrafos tais como propriedade $Z$ e crescimento exponencial, na qual também será apresentado o Teorema 3.21 exibido acima. No capítulo 4 estudaremos propriedades de dígrafos de Cayley de grupos finitamente gerados com crescimento polinomial obtidas através das relações de alcance, em particular apresentamos o Teorema 4.15 exibido anteriormente, que é o principal resultado deste trabalho. Finalizaremos este capítulo com uma conjectura, a qual se for verdadeira fornece uma resposta positiva para o seguinte problema proposto por Grigorchuck:

Problema 4.2. Todo grupo infinito simples e finitamente gerado tem crescimento exponencial? 


\section{Capítulo \\ Preliminares}

Neste capítulo faremos uma breve revisão de algumas definições e resultados da teoria de grupos, que serão usados ao longo do trabalho. Alguns resultados apresentados aqui já fazem parte de qualquer curso básico de grupos, sendo assim algumas demonstrações serão omitidas. Para um maior aprofundamento no assunto, indicamos [9], [7] e [17].

Seja $G$ um grupo e seja $\Omega$ um conjunto não vazio. Suponha que para cada $g \in G$ e $\omega \in \Omega$ definimos um elemento ${ }^{g} \omega \in \Omega$. Dizemos que isto define uma ação de $G$ sobre $\Omega$ (ou $G$ age sobre $\Omega$ ou que $\Omega$ é um $G$-espaço), se valem as seguintes condições:

i) ${ }^{1} \omega=\omega$, para todo $\omega \in \Omega$, onde 1 denota a identidade de $G$.

ii) $\quad g\left({ }^{h} \omega\right)={ }^{g h} \omega$, para todo $\omega \in \Omega$ e todo $g, h \in G$.

Exemplo 1.1. O grupo simétrico $G=\operatorname{Sym}(\Omega)$ (é o conjunto de todas as bijeções de $\Omega$ em $\Omega$ munido da operação composição $\circ)$ tem uma ação natural sobre $\Omega$, pois cada elemento de $G$ é uma permutação de $\Omega$, ou seja, o elemento ${ }^{g} \omega:=g(\omega)$ é a imagem por $g$ de $\omega$. Assim

i) ${ }^{e} \omega=e(\omega)=\omega$, onde e é a identidade de $\operatorname{Sym}(\Omega)$.

ii) $g\left({ }^{h} \omega\right)=g(h(\omega))=g(h(\omega))=g \circ h(\omega)={ }^{g h} \omega$.

Exemplo 1.2. Considere $\Omega=G$ e para cada $x \in G$ defina um elemento ${ }^{g} x:=g x$, onde $g \in G$. Então temos que

i) ${ }^{1} x=x$, onde 1 é a identidade de $G$.

ii) ${ }^{g h} x=(g h) x=g(h x)=g\left({ }^{h} x\right)={ }^{g}\left({ }^{h} x\right)$, para todo $g, h \in G$.

Logo, $G$ age sobre $\Omega$. Tal ação é dita uma ação por multiplicação à esquerda (ou que G age por multiplicação à esquerda). 
Sejam $G$ um grupo, $\Omega$ um $G$-espaço e $\omega \in \Omega$. Definimos a órbita de $\omega$, como o conjunto

$$
{ }^{G} \omega:=\left\{{ }^{g} \omega \mid g \in G\right\}
$$

E o estabilizador de $\omega$ em $G$, é o conjunto

$$
G_{w}:=\left\{\left.g \in G\right|^{g} \omega=\omega\right\}
$$

É fácil verificar que $G_{\omega}$ é subgrupo de $G$.

Dizemos que a ação de $G$ sobre $\Omega$ é transitiva (ou que $\Omega$ é um $G$-espaço transitivo) se para todo $x, y \in \Omega$ existe $g \in G$ tal que ${ }^{g} x=y$, ou equivalentemente, se $G$ age com uma única órbita sobre $\Omega$. Se além disso, tal elemento é único, então a ação é dita regular (ou $G$ age regularmente sobre $\Omega$ ). Logo, a ação é regular se é transitiva e $G_{x}=\{1\}$ para todo $x \in \Omega$.

A demonstração do teorema abaixo pode ser encontrado em [[7], Teorema 1.4A].

Teorema 1.3. Suponha que $G$ age sobre um conjunto $\Omega$ e que $x, y \in \Omega$ e $g \in G$. Então

i) Duas órbitas ${ }^{G} x$ e ${ }^{G} y$ são iguais (como conjuntos) ou são disjuntas, logo o conjunto de todas as órbitas é uma partição de $\Omega$.

ii) O estabilizador $G_{x}=g G_{y} g^{-1}$ sempre que $x={ }^{g} y$, para todo $x, y \in \Omega$ e $g \in G$.

iii) $\left|{ }^{G} x\right|=\left|G: G_{x}\right|$, onde $x$ é um elemento qualquer de $\Omega$. Em particular, se $G$ age regularmente sobre $\Omega$ então $|G|=|\Omega|$.

Observação 1.4. Se $G \leq A u t(\Omega)$ (conjunto de todos automorfismo de $\Omega$ em $\Omega$ munido da operação composição de funções) é um grupo abeliano agindo transitivamente sobre $\Omega$ então $G$ age regularmente sobre $\Omega$. De fato, como $G$ age transitivamente, então resta provarmos que o estabilizador $G_{x}=\{1\}$, para todo $x \in \Omega$. Como $G$ é abeliano, temos pelo Teorema 1.3 que os estabilizadores elementos de $\Omega$ coincidem. Deste modo se $g \in G$ fixa algum elemento de $\Omega$, entâa $g$ fixa todos elementos de $\Omega$. Como o único automorfismo que fixa todos os elementos de $\Omega$ é a identidade, segue que $G_{x}=\{1\}$, para todo $x \in \Omega$.

Como $\Omega$ é um $G$-espaço, para cada $g \in G$, defina uma aplicação

$$
\begin{aligned}
\rho_{g}: \Omega & \rightarrow \Omega \\
\omega & \rightarrow{ }^{g} \omega .
\end{aligned}
$$

Note que tal aplicação define uma ação de $G$ sobre $\Omega$. Agora, provaremos que a aplicação acima é uma bijeção, mostrando que cada $\rho_{g}$ tem um inverso, onde $g \in G$. Sejam $g, h \in G$ e $\omega \in \Omega$, então

$$
\left(\rho_{g} \circ \rho_{h}\right)(\omega)=\rho_{g}\left(\rho_{h}(\omega)\right)=\rho_{g}\left({ }^{h}(\omega)\right)={ }^{g}\left({ }^{h}(\omega)\right)={ }^{g h} \omega .
$$


A última igualdade é obtida do item (ii) da definição de ação. Assim, $\left(\rho_{g} \circ \rho_{h}\right)=\rho_{g h}$. Em particular para $h=g^{-1}$ temos:

$$
\rho_{g} \circ \rho_{g^{-1}}=\rho_{g g^{-1}}=\rho_{e}=\rho_{g^{-1} g}=\rho_{g^{-1}} \circ \rho_{g}
$$

E isto implica que $\left(\rho_{g}\right)^{-1}=\rho_{g^{-1}}$, como queríamos. Portanto, $\rho_{g} \in \operatorname{Sym}(\Omega)$. Segue que temos uma aplicação

$$
\begin{aligned}
\rho: G & \rightarrow \operatorname{Sym}(\Omega) \\
g & \rightarrow \rho(g):=\rho_{g}
\end{aligned}
$$

Além disso, $\rho$ é um homomorfismo. De fato, sejam $g, h \in G$, então

$$
\rho(g h)=\rho_{g h}=\rho_{g} \rho_{h}=\rho(g) \rho(h) .
$$

Denotaremos por $i d$ o elemento identidade de $\operatorname{Sym}(\Omega)$. O núcleo de $\rho$, dado por

$$
\operatorname{ker} \rho=\left\{g \in G \mid \rho_{g}=i d\right\}=\left\{g \in G \mid \rho_{g}(\omega)=i d, \forall \omega \in \Omega\right\}=\left\{g \in G \mid{ }^{g} \omega=\omega, \forall \omega \in \Omega\right\} \text {, }
$$

é um subgrupo normal de $G$ e pelo $1^{\circ}$ Teorema do isomorfismo de grupos:

$$
\frac{G}{\operatorname{ker} \rho} \cong \operatorname{Im} \rho \leq \operatorname{Sym}(\Omega)
$$

onde $\operatorname{Im} \rho$ denota a imagem de $\rho$. Dizemos que $\rho(G)=\operatorname{Im} \rho$ é o grupo de permutação induzido por $G$ em $\Omega$. Em particular, se ker $\rho=\{1\}$, dizemos que a ação de $G$ sobre $\Omega$ é fiel, ou que $G$ age fielmente. E neste caso, podemos pensar em $G$ como um grupo de permutação em $\Omega$. Caso contrário, $G$ é um grupo de permutação módulo ker $\rho$. E temos também que $\frac{G}{\operatorname{ker} \rho}$ age fielmente sobre $\Omega$.

Assuma agora que o conjunto $\Omega$ é um $G$-espaço transitivo. Seja $\approx$ uma relação de equivalência sobre $\Omega$. Se $\approx$ é invariante pela ação de $G$, ou seja,

$$
x \approx y \Leftrightarrow{ }^{g} x \approx{ }^{g} y
$$

para todo $x, y \in \Omega$ e $g \in G$, dizemos que $\approx$ é uma $G$-congruência sobre $\Omega$. Uma $G$-congruência é dita não trivial se existe uma classe de equivalência com mais de um elemento, e é dita própria se existe mais do que uma classe de equivalência.

Exemplo 1.5. Para $G:=G L(2, \mathbb{R})$ agindo sobre $\mathbb{R}^{2} \backslash\{(0,0)\}$, a relação 
é uma G-congruência. Se identificarmos $\mathbb{R}^{2}$ com o plano, então as $\approx$-classes são retas passando pela a origem mas não contendo a origem.

A relação entre subgrupo normal de um grupo transitivo $G$ e uma $G$-congruência é dada pelo seguinte teorema.

Teorema 1.6. Seja $\Omega$ um G-espaço transitivo e seja $H$ um subgrupo normal de $G$. Então as órbitas de $H$ são as classes de uma G-congruência sobre $\Omega$.

Demonstração. Sejam $x, y \in \Omega$. Defina a seguinte relação

$$
x \approx y \Leftrightarrow \text { existe algum } h \in H \text { tal que }{ }^{h} x=y .
$$

Note que $\approx$ é uma relação de equivalência e as classes de equivalências são as órbitas de $H$.

Suponha que $x \approx y$, logo existe $h \in H$ tal que ${ }^{h} x=y$. Queremos mostrar que ${ }^{g} x \approx{ }^{g} y$, $g \in G$. Veja que, ${ }^{g} y={ }^{g h} x$. Como $H \unlhd G$ então existe algum $h^{\prime} \in H$ tal que $g h=h^{\prime} g$. Assim, ${ }^{g} y={ }^{g h} x={ }^{h} g x$, o que implica em ${ }^{g} x \approx{ }^{g} y$, pois $h^{\prime} \in H$.

Agora, se ${ }^{g} x \approx{ }^{g} y$ então ${ }^{g} y={ }^{h g} x$, para algum $h \in H$, logo $y=g^{-1} h g x$ e, portanto $x \approx y$, pois $g^{-1} h g \in H$.

Definição 1.7. Seja $\Omega$ um $G$-espaço transitivo. Se não existem $G$-congruências próprias e nãotriviais então dizemos que $\Omega$ é um G-espaço primitivo ou que G age primitivamente sobre $\Omega$. Caso contrário, dizemos que $G$ age imprimitivamente sobre $\Omega$.

A definição acima implica que se $\approx$ é uma $G$-congruência sobre um $G$-espaço primitivo, então $\approx$ é trivial (todas as classes tem somente um elemento) ou universal (existe somente uma classe, todo $\Omega)$.

Definição 1.8. Um subconjunto não vazio $\Delta \subseteq \Omega$ é um bloco se para todo $g \in G$, ou $\Delta \cap{ }^{g} \Delta=\emptyset$ ou $\Delta={ }^{g} \Delta$. Dizemos que um bloco é não trivial se $|\Delta|>1$, e próprio se $\Delta \neq \Omega$.

Exemplo 1.9. Se $G$ age transitivamente sobre $\Omega$ e se $\Delta$ e $\Gamma$ são blocos de $G$ contendo um ponto em comum então $\Delta \cap \Gamma$ é também um bloco de $G$. 
Definição 1.10. Seja $G$ um grupo agindo transitivamente sobre $\Omega$. Seja $\Delta$ um bloco. O sistema de blocos contendo $\Delta$, é o conjunto definido por

$$
\Sigma:=\left\{{ }^{x} \Delta \mid x \in G\right\}
$$

Um sistema de blocos $\Sigma$ é dito sistema de imprimitividade se $\Sigma$ é formado por blocos não triviais. Observe que um sistema de blocos $\Sigma$ é uma partição de $\Omega$ e cada elemento de $\Sigma$ é um bloco.

Definição 1.11. Seja $\Delta$ um bloco. O estabilizador de $\Delta$ é o conjunto de elementos de $G$ que deixa $\Delta$ invariante, isto é,

$$
G_{\{\Delta\}}:=\left\{g \in G \mid{ }^{g} \Delta=\Delta\right\}
$$

Note que $G_{\{\Delta\}}$ é um subgrupo de $G$.

Lema 1.12. Seja $G$ um grupo agindo transitivamente sobre um conjunto $\Omega$ e seja $\Delta$ um bloco para $G$. Então $G_{\{\Delta\}}$ age transitivamente sobre $\Delta$.

Demonstração. Sejam $x, y \in \Delta$. Pela transitividade de $G$ existe $g \in G$ tal que $y={ }^{g} x$. Assim, $y \in \Delta$ e $y={ }^{g} x \in{ }^{g} \Delta$, isto é, $\Delta \cap{ }^{g} \Delta$, de onde concluímos que $\Delta={ }^{g} \Delta$, pois $\Delta$ é um bloco. Portanto $g \in G_{\{\Delta\}}$.

Para finalizar este capítulo daremos agora algumas definições básicas de grupos abstratos.

Sejam $G$ um grupo e $X$ um subconjunto não vazio de $G$. Denotamos por $\langle X\rangle$ o subgrupo de $G$ gerado por $X$. Se $X$ é finito e gera $G, G=\langle X\rangle$, então dizemos que $G$ é finitamente gerado.

Uma palavra sobre $X$, de comprimento $n$, é um elemento $\omega \in G$ da forma

$$
\omega=x_{1}^{e_{1}} x_{2}^{e_{2}} \ldots x_{n}^{e_{n}}
$$

onde $x_{i} \in X, e_{i} \in\{-1,1\}$ e $n \geq 1$.

Sejam $x, y \in G$. O comutador de $x$ e $y$ é definido como $[x, y]=x^{-1} y^{-1} x y$. Sejam $H, K$ subgrupos de $G$, definimos o comutador de $H$ e $K$ como sendo o subgrupo

$$
[H, K]:=\langle[h, k]| h \in H \text { e } k \in K\rangle
$$


Além disso, se $H=K=G$, então $G^{\prime}=[G, G]$ é dito subgrupo derivado de $G$.

Definição 1.13. A série central inferior de um grupo $G$ é definida por:

$$
\begin{aligned}
\gamma_{1}(G) & :=G \\
\gamma_{2}(G) & :=\left[\gamma_{1}(G), G\right] \\
& \vdots \\
\gamma_{r}(G) & :=\left[\gamma_{r-1}(G), G\right]
\end{aligned}
$$

onde $r \geq 1$.

Dizemos que $G$ é nilpotente de classe $r$, se $r$ é o menor número natural tal que $\gamma_{r+1}(G)=1$.

A demonstração do próximo teorema pode ser encontrada em ([17], Teorema 5.36)

Teorema 1.14. Se $G$ é nilpotente de classe $r$ e $H \unlhd G$ então $G / H$ é nilpotente de classe menor ou igual à $r$. 


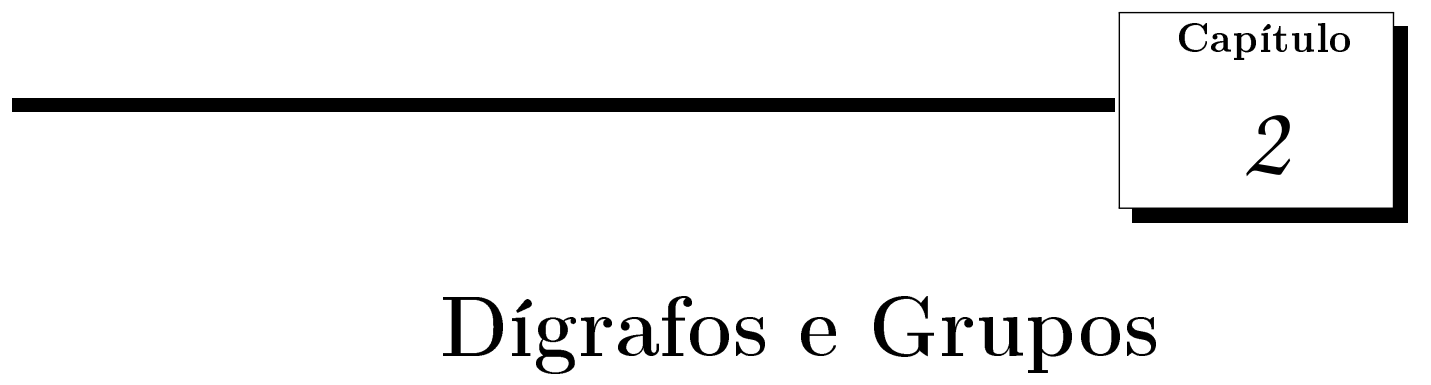

Neste capítulo, inicialmente relembraremos algumas definições e resultados básicos da teoria dos dígrafos. Um destes mostra que em um dígrafo infinito transitivo e conexo sempre existe um passeio direcionado de comprimento arbitrariamente grande iniciando em qualquer vértice. $\mathrm{Na}$ Seção 2.2 definiremos propriedade $Z$ e provaremos que um dígrafo $D$ infinito, conexo, e transitivo, tem propriedade $Z$ se, e somente se, todo ciclo de $D$ é balanceado (veja Lema 2.4). Na Seção 2.3 estudaremos dígrafos de Cayley, em particular, apresentaremos um lema que nos dá uma condição necessária e suficiente para um dígrafo ser um dígrafo de Cayley. Finalizaremos este capítulo com uma seção sobre crescimento de dígrafos.

\subsection{Definições}

Um dígrafo $D$ é um par ordenado $(V D, E D)$, onde $V D$ é um conjunto não vazio e $E D \subseteq V D \times V D$, conjunto de pares ordenados de $V D$. Os elementos de $V D$ são chamados de vértices e os elementos de $E D$ de arestas do dígrafo $D$. Note que um dígrafo pode conter laços $(v, v)$, bem como pares de arestas opostas $(u, v)$ e $(v, u)$. Enfatizamos que com esta definição os dígrafos considerados neste trabalho são sempre simples, no sentido que entre dois vértices existe no máximo uma aresta em cada direção. Dizemos que $D^{\prime}=\left(V D^{\prime}, E D^{\prime}\right)$ é um subdígrafo de $D$ se $V D^{\prime} \subseteq V D$ e $E D^{\prime} \subseteq E D$.

Dado um dígrafo $D$, dizemos que $D$ é finito se o conjunto $V D$ é finito, caso contrário, dizemos 
que $D$ é infinito.

Exemplo 2.1. A Figura 2.1 apresenta um dígrafo finito com conjunto de vértices

$$
V D=\{1,2,3,4,5,6,7\}
$$

e conjunto de arestas

$$
E D=\{(1,2),(2,3),(3,4),(4,5),(5,6),(6,7),(7,1)\}
$$

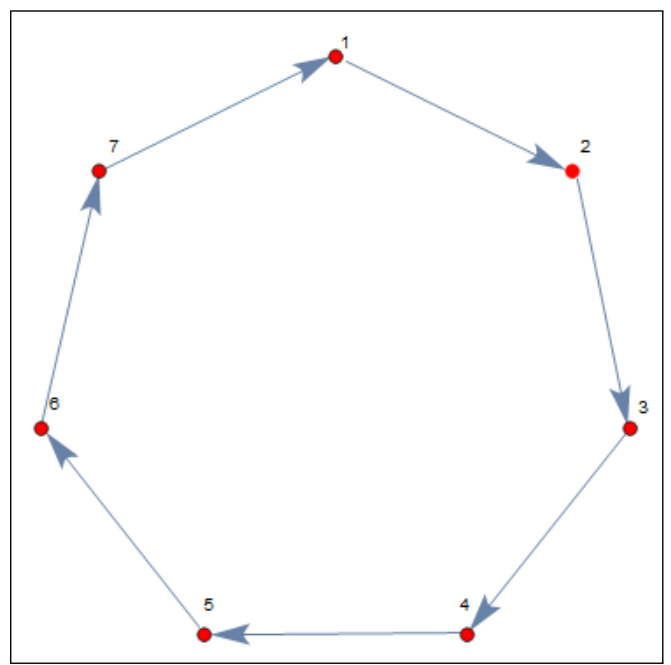

Figura 2.1: exemplo de dígrafo

Exemplo 2.2. O dígrafo $D$ da Figura 2.2 é infinito, tem como conjunto de vértices o conjunto dos números inteiros $\mathbb{Z}$ e conjunto de arestas $E D=\{(i, i+1) \mid i \in \mathbb{Z}\}$. O dígrafo $D$ é conhecido como dígrafo $Z$ ou dígrafo inteiro.

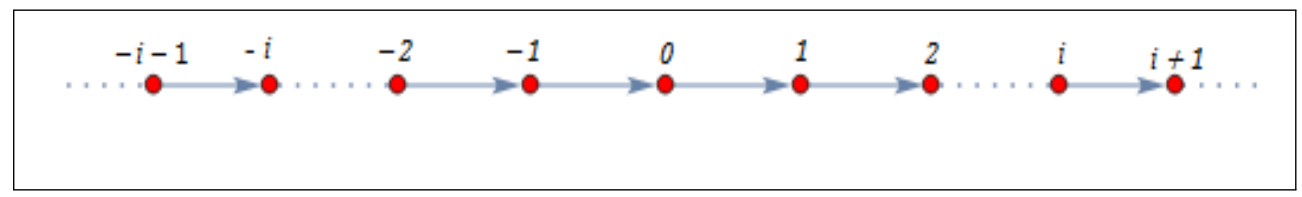

Figura 2.2: dígrafo $\mathrm{Z}$

Dado um dígrafo $D$, o grafo correspondente de $D$ é a estrutura obtida de $D$ desconsiderando as orientações das arestas. Dizemos que dois vértices são adjacentes se eles são ligados por uma aresta. Dado um vértice $v \in V D$, os vizinhos de $v$ são todos os vértices adjacentes a $v$. 
Um passeio $W=\left(v_{0}, \epsilon_{1}, v_{1}, \ldots, \epsilon_{n}, v_{n}\right)$ de $v_{0}$ a $v_{n}$, de comprimento $|W|=n$, é uma sequência de $n+1$ vértices (não necessariamente distintos) $v_{0}, v_{1}, \ldots, v_{n}$ e de $n$ indicadores $\epsilon_{1}, \epsilon_{2}, \ldots, \epsilon_{n} \in$ $\{1,-1\}$ tal que para todo $j \in\{1,2, \ldots, n\}$ temos:

$$
\begin{gathered}
\epsilon_{j}=1 \Rightarrow\left(v_{j-1}, v_{j}\right) \in E W \\
\epsilon_{j}=-1 \Rightarrow\left(v_{j}, v_{j-1}\right) \in E W .
\end{gathered}
$$

Intuitivamente, um passeio é um percurso no dígrafo de vértice em vértice ao longo das arestas, onde os indicadores 1 e -1 informam quando o percurso respeita, ou não, o sentido da aresta, respectivamente.

Dizemos que $v_{0}$ é o vértice inicial de $W$ e $v_{n}$ é vértice final de $W$. Em particular, $W$ é um passeio fechado se $v_{0}=v_{n}$.

Um passeio é direcionado se todos os indicadores de $W$ são iguais à 1 (ou -1), e ele é alternado se os valores dos indicadores alternam. Dado um passeio $W=\left(v_{0}, \epsilon_{1}, v_{1}, \ldots, \epsilon_{n}, v_{n}\right)$, o passeio inverso de $W$ é $W^{-1}=\left(v_{n},-\epsilon_{n}, v_{n-1}, \ldots,-\epsilon_{1}, v_{0}\right)$. Para $0 \leq i \leq j \leq n$, a subsequência

$$
{ }_{i} W_{j}=\left(v_{i}, \epsilon_{i+1}, v_{i+1}, \ldots, \epsilon_{j}, v_{j}\right)
$$

é dita um subpasseio de $W$. Além disso, se

$$
W^{\prime}=\left(u_{0}, \delta_{1}, u_{1}, \ldots, \delta_{m}, u_{m}\right)
$$

é um passeio tal que $u_{0}=v_{n}$, então a concatenação de $W$ e $W^{\prime}$ é o passeio

$$
W . W^{\prime}=\left(v_{0}, \epsilon_{1}, v_{1}, \ldots, \epsilon_{n}, u_{0}, \delta_{1}, u_{1}, \ldots, \delta_{m}, u_{m}\right)
$$

de comprimento $n+m$. Temos também que o peso de $W$, denotado por $F(W)$, é

$$
F(W)=\epsilon_{1}+\epsilon_{2}+\cdots+\epsilon_{n}
$$

isto é, a soma dos seus indicadores. Se $F(W)=0$ então dizemos que $W$ é balanceado. 
Um caminho é um passeio $W$ cujos vértices são dois a dois distintos. Dizemos que o dígrafo $D$ é conexo se para todo par de vértices $u, v \in V D$ existe um caminho ligando $u$ a $v$. Caso contrário, dizemos que $D$ é desconexo. Um ciclo $C$ é um caminho cujos extremos coincidem. Se $D$ é conexo e não contém ciclos, então dizemos que $D$ é uma árvore.

Seja $D$ um dígrafo. Dado $v \in V D$ e $j \in \mathbb{N}$. Definimos $D_{j}^{+}(v)$ como sendo o conjunto de vértices finais de todos passeios direcionados de comprimento $j$ com vértice inicial $v$ e $D_{j}^{-}(v)$ denota o conjunto dos vértices iniciais de todos os passeios direcionados de comprimento $j$ com vértice final $v$. Se $S \subset V D$ então

$$
D_{j}^{+}(S)=\bigcup_{v \in S} D_{j}^{+}(v) \text { e } D_{j}^{-}(S)=\bigcup_{v \in S} D_{j}^{-}(v)
$$

O grau de saída $d^{+}(v)$ de $v$ é a cardinalidade do conjunto $D_{1}^{+}(v)$. E o grau de entrada $d^{-}(v)$ de $v$ é a cardinalidade de $D_{1}^{-}(v)$. Se os graus de saída (respectivamente de entrada) são os mesmos para todo vértice de $D$, escreveremos apenas $d_{D}^{+}$(respectivamente $d_{D}^{-}$). O subíndice $D$ é omitido se $D$ está claro no contexto. Um dígrafo é dito regular se $d^{+}(v)=d^{+}\left(v^{\prime}\right)$ e $d^{-}(v)=d^{-}\left(v^{\prime}\right)$, para todo $v, v^{\prime} \in V D$.

Um dígrafo $D$ é localmente finito se ambos $d^{+}(v)$ e $d^{-}(v)$ são finitos para todo $v \in V D$. Caso contrário diremos que $D$ é localmente infinito.

Antes de finalizarmos esta seção, definiremos automorfismo de dígrafos e, consequentemente, a definição de dígrafo transitivo.

Sejam $D$ e $D^{\prime}$ dígrafos. Um homomorfismo de dígrafos $\phi: D \rightarrow D^{\prime}$ é uma aplicação $\phi: V D \rightarrow V D^{\prime}$ que preserva aresta, isto é,

$$
(u, v) \in E D \Rightarrow(\phi(u), \phi(v)) \in E D^{\prime}
$$

Se $\phi$ é sobrejetora então $\phi$ é um epimorfismo. Se $\phi$ é bijetor e a função inversa preserva aresta, então $\phi$ é um isomorfismo e dizemos que $D$ e $D^{\prime}$ são isomorfos. E, se $\phi$ é um isomorfismo e $D=D^{\prime}$, então $\phi$ é um automorfismo de dígrafos.

O conjunto de todos os automorfismos de um dígrafo é um grupo com a operação de composição de funções e tal grupo é denotado por $A u t(D)$. Se existe $G \leq A u t(D)$ agindo transitivamente 
sobre $V D$, dizemos que o dígrafo $D$ é transitivo. Observe que a ação de $G$ sobre $V D$ induz uma ação natural sobre $E D$ dada por:

$$
{ }^{g}(u, v):=\left({ }^{g} u,{ }^{g} v\right)
$$

para todo $(u, v) \in E D$ e $g \in G$.

Lema 2.3. Seja $D$ um dígrafo infinito, conexo e transitivo. Então existem em $D$ caminhos direcionados de comprimento arbitrariamente grande .

Demonstração. Se $D$ é localmente finito, a existência de caminhos direcionados de comprimento arbitrariamente grande é garantida pelo resultado de Trofimov em [19].

Se $D$ é localmente infinito, então pelo menos $d^{+}$ou $d^{-}$é infinito. Suponha que $d^{+}$é infinito e seja $v_{1} \in V D$. Da suposição e de $D$ conexo, existe $v_{2} \in V D$ tal que $\left(v_{1}, v_{2}\right) \in E D$. Escolha agora um $v_{3} \in V D$ tal que $\left(v_{2}, v_{3}\right) \in E D$, onde $v_{1} \neq v_{2} \neq v_{3}\left(v_{3}\right.$ existe pois $d^{+}$é infinito, isto é, podemos sempre escolher vértices distintos). Repetindo este processo quantas vezes forem necessárias, obtemos o caminho desejado. De fato, uma vez que em cada passo temos infinitas opções de escolhas de vértices e após tal passo temos somente um número finito de vértices visitados. De forma análoga prova-se o caso $d^{-}=\infty$.

\subsection{Propriedade $\mathrm{Z}$}

Seja $D$ um dígrafo. Dizemos que $D$ tem propriedade $Z$ se existe um epimorfismo $\phi: D \rightarrow Z$, onde $Z$ é o dígrafo apresentado no Exemplo 2.2.

Para um dígrafo conexo, infinito e transitivo $D$ ter a propriedade $\mathrm{Z}$ é equivalente a afirmar que todos os ciclos de $D$ são balanceados, conforme veremos no resultado abaixo.

Lema 2.4. Seja $D$ um dígrafo infinito, conexo e transitivo. Então $D$ tem propriedade $Z$ se, e somente se, todo ciclo de D é balanceado.

Demonstração. Suponha que $D$ tem propriedade Z. Então existe um homomorfismo sobrejetor $\phi: D \rightarrow Z$. Seja $C=\left(v_{0}, \epsilon_{1}, v_{1}, \ldots, \epsilon_{n}, v_{n}\right)$ um passeio simples fechado de $D$. Como $\phi$ é um homomorfismo, então

$$
\phi\left(v_{n}\right)=\phi\left(v_{0}\right)+\sum_{i=1}^{n} \epsilon_{i}=\phi\left(v_{0}\right)+F(C) .
$$


Como $C$ é um passeio fechado segue que $v_{0}=v_{n}$. Logo $\phi\left(v_{n}\right)=\phi\left(v_{0}\right)$ e, portanto $F(C)=0$.

Reciprocamente, fixe $u \in V D$. Defina uma aplicação $\phi: V D \rightarrow V Z$ tal que $\phi(u)=0 \mathrm{e}$ $\phi(v):=F(W)$, onde $W$ é um passeio de $u$ até $v$. Observe que, se existem dois caminhos distintos ligando $u$ à $v$, digamos $W_{1}$ e $W_{2}$, então o passeio $W=W_{1} \cdot\left(W_{2}\right)^{-1}$ é formado pela união de passeios simples fechados $C_{1}, C_{2}, \ldots, C_{n}$. Logo, $F(W)=0$ pois $F\left(C_{i}\right)=0$ por hipótese. Portanto $F\left(W_{1}\right)=-F\left(W_{2}^{-1}\right)=F\left(W_{2}\right)$, e segue que $\phi$ está bem definida. Agora, seja $(x, y) \in E D$. Por definição, $\phi(y)=\phi(x)+1$. Como $\phi(x)=i$ para algum $i \in \mathbb{Z}$, segue que $\phi(y)=i+1$, e portanto, $(\phi(x), \phi(y))=(i, i+1) \in E Z$. Logo, $\phi$ é um homomorfismo. Finalmente, o fato de $\phi$ ser sobrejetora segue do Lema 2.3 .

Exemplo 2.5. Uma árvore infinita regular (com grau de entrada e grau de saída constante) claramente tem a propriedade $Z$, pois não contém ciclos.

Em [16], Praeger dá uma condição para um dígrafo conexo, infinito, transitivo nos vértices e nas arestas ter propriedade $Z$. Como podemos ver a seguir.

Teorema. Seja D um dígrafo infinito, conexo, transitivo nos vértices e nas arestas e com grau de entrada e grau de saída ambos finitos mas distintos. Então D tem propriedade $Z$.

\subsection{Dígrafos de Cayley}

O objetivo principal desta seção será discutir alguns resultados básicos sobre dígrafos de Cayley. O dígrafo de Cayley $\operatorname{Cay}(G, S)$ de um grupo $G$ com respeito à um subconjunto $S$ de $G \backslash\{1\}$, onde 1 denota a identidade de $G$, tem como conjunto de vértices o grupo $G$ e o conjunto de arestas $\operatorname{ECay}(G, S)=\{(g, g s) \mid s \in S\}$.

Note que $G$ age regularmente sobre $D=\operatorname{Cay}(G, S)$ por multiplicação à esquerda. Dados $g, x \in G,{ }^{x} g:=x g$, para todo $g \in G$. Consequentemente, temos uma ação natural de $G$ sobre as arestas de $D$ dada por:

$$
{ }^{x}(g, g s)=\left({ }^{x} g,\left({ }^{x} g\right) s\right)=(x g, x g s),
$$

onde $x \in G$. Como $(x g, x(g s)) \in E D$, segue que $G$ preserva aresta e, portanto $G$ é um subgrupo de $\operatorname{Aut}(D)$ agindo regularmente sobre $V D$. 
Lema 2.6. Seja $D=\operatorname{Cay}(G, S)$ um dígrafo de Cayley com respeito ao subconjunto $S$ de $G$. Então $D$ é conexo se, e somente se, $S$ é um conjunto de geradores de $G$.

Demonstração. Primeiramente observe que qualquer caminho em $D$ cujo vértice inicial é a identidade, tem como vértice final uma palavra em $S$. Suponha que $S$ é um conjunto de geradores de $G$. Então para quaisquer dois vértices $u$ e $v$ de $D$, temos que $u$ e $v$ são palavras em $S$. Logo, existem em $D$ um caminho $W$ da identidade até $u$, e um caminho $W^{\prime}$ da identidade até $v$. Assim, a concatenação $W^{-1} \cdot W^{\prime}$ é um passeio de $u$ a $v$. Como, $u$ e $v$ são arbitrários, segue que $D$ é conexo.

Reciprocamente, seja $v \in V D$. Temos que mostrar que $v$ é uma palavra em $S$. Como $D$ é conexo, existe um caminho com vértice inicial igual a identidade e vértice final $v$, mas pela observação acima temos que $v$ é uma palavra em $S$. Como $v$ é arbitrário, então $S$ é um conjunto de geradores para $G$.

Enfatizamos que, salvo menção contrária, estaremos considerando que $\operatorname{Cay}(G, S)$ é um dígrafo de Cayley de $G$ com respeito ao conjunto de geradores $S$ de $G$.

Exemplo 2.7. $\mathrm{Na}$ Figura 2.3 apresentamos o digrafo de Cayley $\mathrm{Cay}\left(S_{3}, S\right)$, onde $S_{3}=\left\langle r, t \mid r^{3}=1, t^{2}=1, r t r=t\right\rangle$ e $S=\{r, t\}$.

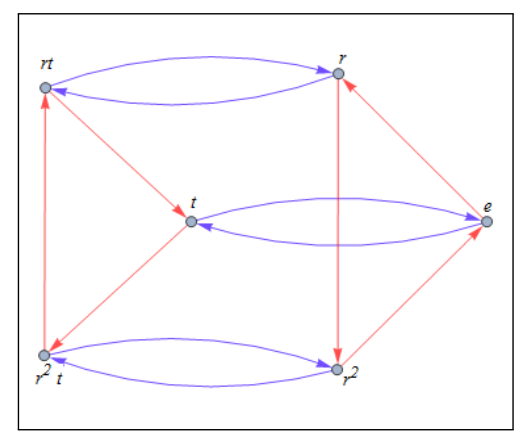

Figura 2.3: $\operatorname{Cay}\left(S_{3}, S\right)$, aresta azul: gerada por $t$; aresta vermelha: gerada por $r$.

Exemplo 2.8. $N a$ figura 2.4 apresentamos o dígrafo de Cayley $C a y\left(D_{4}, S\right)$, onde $D_{4}=\left\langle r, t \mid r^{4}=1, t^{2}=1, r t r=t\right\rangle$ e $S=\{r, t\}$. 


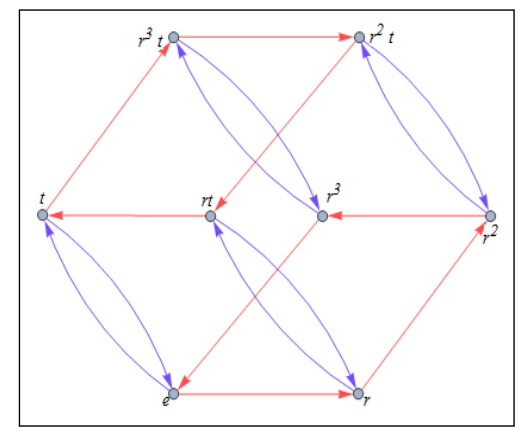

Figura 2.4: $\operatorname{Cay}\left(D_{4}, S\right)$, aresta azul: gerada por $t$; aresta vermelha: gerada por $r$.

Lema 2.9. Seja $D$ um dígrafo conexo. Suponha que existe um subgrupo $G \operatorname{de} A u t(D)$ agindo regularmente sobre $D$. Então $D$ é isomorfo ao dígrafo de Cayley $C a y(G, S)$ de $G$ para algum conjunto $S$ de geradores de $G$.

Demonstração. Fixe $v \in V D$. Como $G$ age regularmente, então para cada $u \in V D$ existe um único $g \in G$ tal que $u={ }^{g} v$. Tome

$$
S=\left\{g \in G \mid\left(v,{ }^{g} v\right) \in E D\right\}
$$

Primeiramente, mostraremos que $S$ gera $G$. Seja $g \in G$ e considere o vértice ${ }^{g} v$. Como $D$ é conexo, existe

$$
P=\left(v_{0}, v_{1}, \ldots, v_{n-1}, v_{n}\right)
$$

um caminho de $v$ até ${ }^{g} v$. Vamos provar por indução sobre o comprimento do caminho $P$, que $g$ é uma palavra em $S$. Se $n=1$, então ${ }^{g} v \in\left\{{ }^{x} v \mid x \in S \cup S^{-1}\right\}$.

Assuma agora $n \geq 2$ e suponha que o resultado seja válido para todo caminho de comprimento $l<n$. Como ${ }_{0} P_{n-1}$ é um caminho de comprimento $n-1$, por indução, $v_{n-1}={ }^{\omega} v$, para alguma palavra $\omega$ em $S$. Com isto temos dois casos. Primeiro, se $\left(v_{n-1},{ }^{g} v\right)=\left({ }^{\omega} v,{ }^{g} v\right)$ é uma aresta em $D$ então $\left(v, \omega^{-1} g v\right)$ também é uma aresta em $D, \operatorname{logo} \omega^{-1} g \in S$. Assim existe $s \in S$ tal que $\omega^{-1} g=s$, e isto implica que $g=\omega s$. Como $s, \omega \in S$, obtemos que $g$ é uma palavra em $S$. Prova-se de forma análoga, se $\left({ }^{g} v, v_{n-1}\right) \in E D$. Como queríamos. 
Seja $D^{\prime}=\operatorname{Cay}(G, S)$ o dígrafo de Cayley de $G$ com respeito à $S$. Defina a aplicação

$$
\begin{aligned}
\phi: V D & \rightarrow V D^{\prime} \\
g_{v} & \rightarrow g
\end{aligned}
$$

Mostraremos agora que $\phi$ é um isomorfismo de dígrafo. Como $G$ age regularmente, segue que $\phi$ é uma bijeção. Pela transitividade de $G$ sobre $V D$, temos que uma aresta em $D$ é da forma $\left({ }^{g} v,{ }^{g s} v\right)$, para algum $g \in G$ e $s \in S$. Como, $\phi\left({ }^{g} v\right)=g$ e $\phi\left({ }^{g s} v\right)=g s$, então $\left(\phi\left({ }^{g} v\right), \phi\left({ }^{g s} v\right)\right)=(g, g s)$ é uma aresta em $D^{\prime}$, portanto $\phi$ é um homomorfismo de dígrafo. De forma similar, obtemos que $\phi^{-1}$ preserva aresta. Isto prova o resultado.

Segue do lema acima que: Um dígrafo conexo D é um dígrafo de Cayley se, e somente se, existe $H \leq A u t(D)$ agindo regularmente sobre $D$.

Corolário 2.10. Seja $G$ um grupo abeliano agindo transitivamente sobre um dígrafo D. Então $D$ é um dígrafo de Cayley de $G$ com respeito a algum subconjunto $S \subseteq G \backslash\{1\}$.

Demonstração. Pela observação $1.4, G$ age regularmente sobre $D$. Se $D$ é conexo, o resultado segue do Lema 2.9.

Agora, suponha que $D$ é desconexo. Dado $u \in V D$, denote por $D_{u}$ a componente de $D$ que contém $u$. Pelo Lema 2.9, $D_{u}=\operatorname{Cay}\left(\left\langle S_{u}\right\rangle, S_{u}\right)$, onde

$$
S_{u}=\left\{g \in G \mid\left(u,{ }^{g} u\right) \in E D\right\}
$$

Agora, seja $v \in V D$ tal que $D_{v} \neq D_{u}$. Como $G$ é transitivo, existe $g \in G$ tal que $v={ }^{g} u$. Seja $\left(v, s^{\prime} v\right) \in E D$, com $s^{\prime} \in S_{v}$, então $\left(v,{ }^{\prime} v\right)=\left({ }^{g} u, s^{\prime} g u\right)$. De $G$ abeliano temos $g s^{\prime}=s^{\prime} g, \operatorname{logo}$

$$
\left(v, s^{\prime} v\right)=\left({ }^{g} u,{ }^{g s^{\prime}} u\right)={ }^{g}\left(u,{ }^{s^{\prime}} u\right)
$$

com isto, $\left(u, s^{\prime} u\right) \in E D$ implica que $s^{\prime} \in S_{u}$. Portanto, $D_{v}$ é uma cópia de $D_{u}$. Como $v$ é arbitrário, segue que todas as componentes de $D$ são cópias de $D_{u}$. Portanto, $D=\operatorname{Cay}\left(G, S_{u}\right)$. 


\subsection{Crescimento}

Neste trabalho, estudaremos o crescimento de dígrafos transitivos. A distância $\operatorname{dist}_{D}(u, v)$ entre dois vértices em um dígrafo conexo e transitivo $D$ é o comprimento do menor caminho ligando $u$ à $v$. Tome $v \in V D$.

A função crescimento $f(n)$, em relação a $v$ com $n \geq 0$, é dada por

$$
f(n)=\left|\left\{u \in V D \mid \operatorname{dist}_{D}(v, u) \leq n\right\}\right| .
$$

Como $D$ é transitivo a função de crescimento não depende da escolha do vértice $v$.

Dizemos que o dígrafo $D$ tem crescimento exponencial se existe uma constante $c>1$ tal que

$$
f(n)>c^{n}
$$

vale para todo $n>0$. E o dígrafo $D$ tem crescimento polinomial se existem constantes positivas $c$ e $d$ tal que

$$
f(n) \leq c n^{d}
$$

vale para todo $n>0$.

Exemplo 2.11. A Figura 2.5 é um pedaço de uma árvore $T$ infinita regular com $d^{+}=1 e$ $d^{-}=2$. Note que T é transitiva (veja [[1], Teorema 5.20]) e temos que

$$
f(1)>2 ; f(2)>2^{2} ; f(3)>2^{3} ; \ldots
$$

Continuando dessa maneira, obtemos que $f(n)>2^{n}$, para todo $n>0$. Portanto, $T$ tem crescimento exponencial.

Observação 2.12. Para cada inteiro $h \geq 0$, o subdígrafo $T^{h}$ de $T$ induzido em $\bigcup_{i \in[0, h]} T_{i}^{-}(v)$ será chamado de árvore binária de altura $h$ com raiz $v:=v_{15}$. Note que

$$
T^{0} \subset T^{1} \subset \ldots \subset T^{h} \subset \ldots
$$




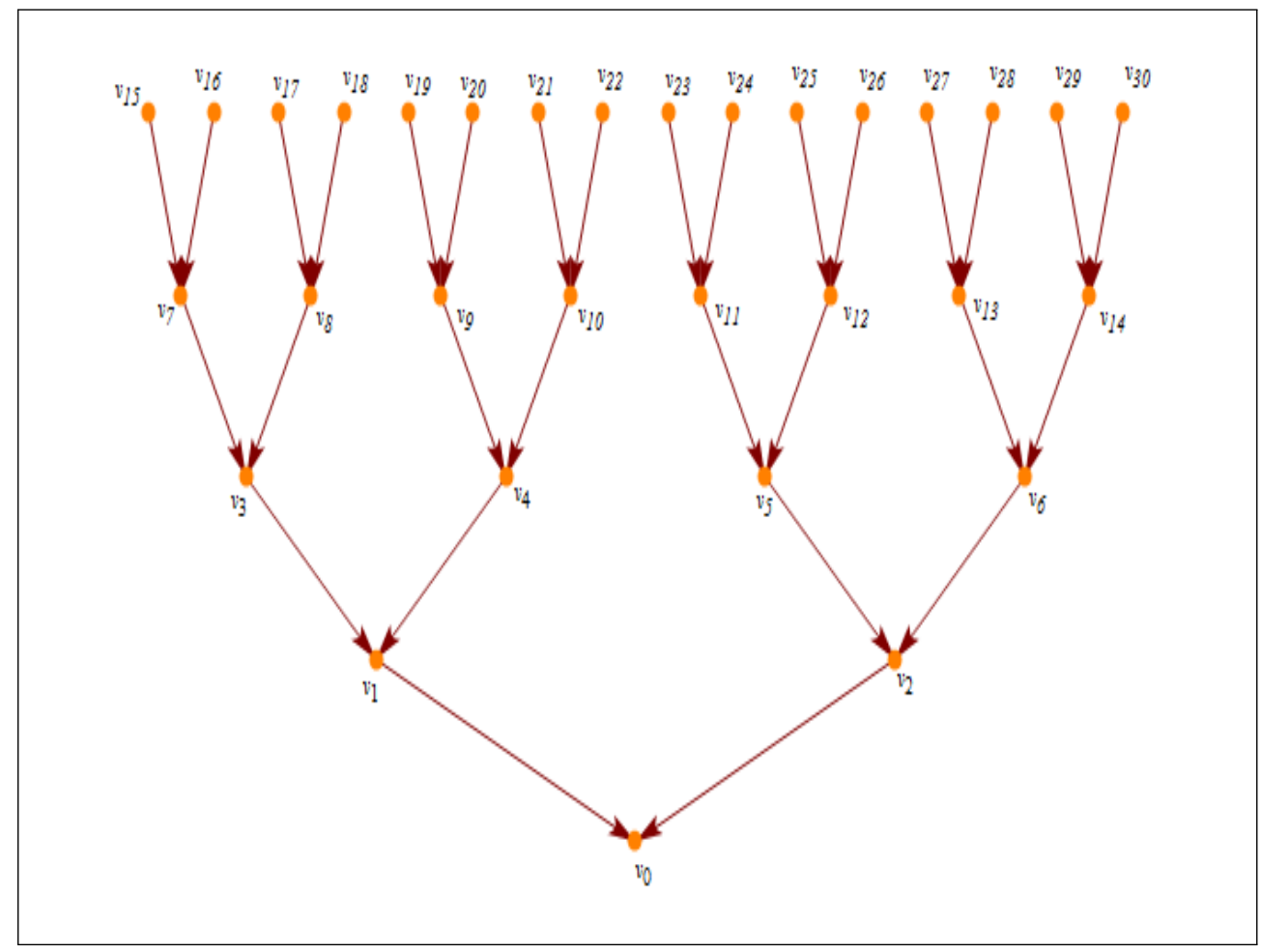

Figura 2.5: pedaço da árvore $T$.

A uni ão $T^{*}:=\bigcup_{i \geq 0} T^{i}$ é uma árvore binária com raiz $v$ e altura arbitrariamente grande.

Exemplo 2.13. A Figura 2.6 apresenta um digrafo $D$ com crescimento polinomial, pois $f(n) \leq 5 n$, para todo $n>0$.

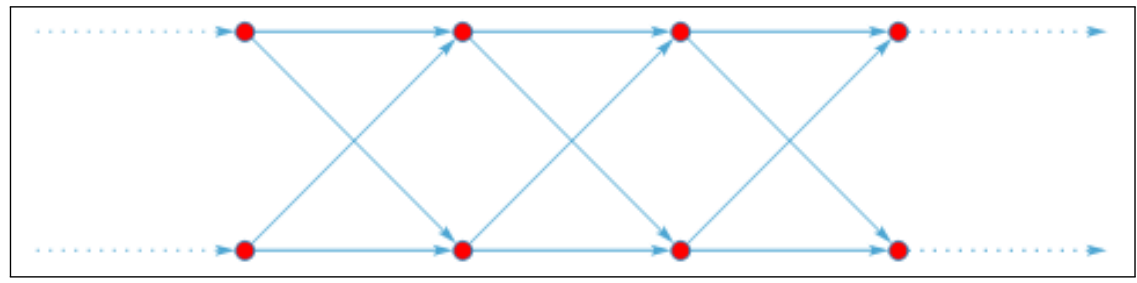

Figura 2.6: dígrafo $D$. 
Para finalizar este capítulo definiremos agora crescimento de grupos. Dizemos que um grupo finitamente gerado $G$ tem crescimento exponencial, se existe um conjunto $S$ de geradores tal que $\operatorname{Cay}(G, S)$ tem crescimento exponencial.

Similarmente definimos crescimento polinomial de um grupo. 


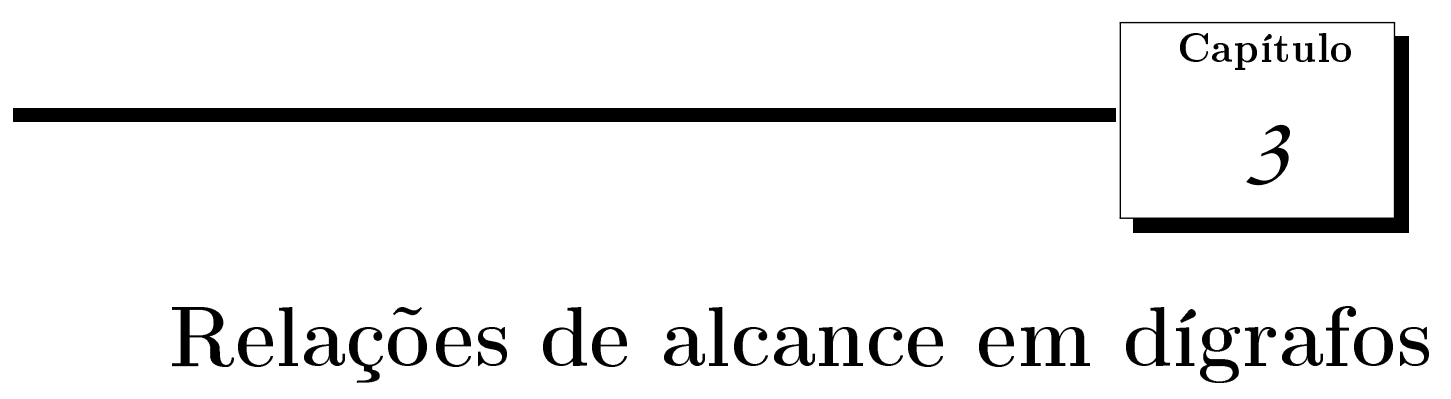

Neste capítulo estudamos uma família de relações de alcance, definida sobre o conjunto de vértices de um dígrafo (possivelmente com laços e pares de vértices ligados por duas arestas opostas), que generaliza um conceito similar introduzido em [15].

Sejam $D$ um dígrafo com grau de entrada e de saída pelo menos igual a 1 e $k \geq 1$ inteiro. Sejam $u, v \in V D$. Dizemos que $u$ está $R_{k}^{+}$-relacionado com $v$, em símbolos $u R_{k}^{+} v$, se existe um passeio $W$ de $u$ a $v$ tal que $F(W)=0$, e para todo $0 \leq j \leq|W|$ temos $F\left({ }_{0} W_{j}\right) \in[0, k]$. O conjunto formado por todos tais passeios será denotado por $R_{k}^{+}[u, v]$. Similarmente, dizemos que $u$ está $R_{k}^{-}$-relacionado com $v$, em símbolos $u R_{k}^{-} v$, se existe um passeio $W$ de $u$ a $v$ tal que $F(W)=0$, e para todo $0 \leq j \leq|W| \operatorname{temos} F\left({ }_{0} W_{j}\right) \in[-k, 0]$. Denotaremos por $R_{k}^{-}[u, v]$ o conjunto de todos tais passeios.

Veremos a seguir que $R_{k}^{+}$e $R_{k}^{-}$são relações de equivalências.

Proposição 3.1. Sejam D um dígrafo com grau de entrada e grau de saída pelo menos 1 e $k \geq 1$ inteiro. Então $R_{k}^{+}$e $R_{k}^{-}$são relaçôes de equivalência. Além disso, se $D$ é transitivo então $R_{k}^{+}$e $R_{k}^{-}$são G-congruências, onde $G \leq A u t(D)$.

Demonstração. Primeiramente provaremos que $R_{k}^{+}$é uma relação de equivalência, isto é, que $R_{k}^{+}$ é reflexiva, simétrica e transitiva. Sejam $u, v$ e $w$ vértices de $D$. Por hipótese, como $D$ tem grau de saída pelo menos 1 , existe $u^{\prime} \in V D$ tal que $\left(u, u^{\prime}\right)$ é uma aresta em $D$. Assim $\left(u, 1, u^{\prime},-1, u\right)$ é um passeio de $R_{1}^{+}[u, u]$. Como $R_{1}^{+} \subseteq R_{k}^{+}$, segue que $R_{k}^{+}$é reflexiva. Agora, suponha que $u R_{k}^{+} v$. 
Sejam $W=\left(u_{0}, \epsilon_{1}, \ldots, \epsilon_{n}, u_{n}\right)$ um passeio pertencente ao conjunto $R_{k}^{+}[u, v]$. Deste modo, o passeio inverso $W^{-1}$ tem peso zero e para todo $0 \leq j \leq n$, temos

$$
F\left({ }_{0}\left(W^{-1}\right)_{j}\right)=-F\left({ }_{n-j} W_{n}\right)=-\left(F(W)-F\left({ }_{0} W_{n-j}\right)\right)=F\left({ }_{0} W_{n-j}\right) .
$$

Com isso, obtemos que $F\left({ }_{0}\left(W^{-1}\right)_{j}\right) \in[0, k]$. Portanto $W^{-1} \in R_{k}^{+}[v, u]$. Finalmente, suponha que $u R_{k}^{+} v$ e $v R_{k}^{+} w$. Sejam os passeios $W \in R_{k}^{+}[u, v]$ e $W^{\prime} \in R_{k}^{+}[v, w]$. Considere o passeio $W^{\prime \prime}=W \cdot W^{\prime}$. Observe que $F\left(W^{\prime \prime}\right)=0$ e para todo $0 \leq j \leq\left|W^{\prime \prime}\right|$, temos

- Se $0 \leq j \leq|W|$, então $F\left({ }_{0} W_{j}^{\prime \prime}\right)=F\left({ }_{0} W_{j}\right) \in[0, k]$.

- Se $|W|<j \leq|W|+\left|W^{\prime}\right|$ então $F\left({ }_{0} W_{j}^{\prime \prime}\right)=F(W)+F\left(|W| W_{j}^{\prime \prime}\right)$, como $|W| W_{j}^{\prime \prime}={ }_{0} W_{j^{\prime}}^{\prime}$, para algum $0 \leq j^{\prime} \leq\left|W^{\prime}\right|$. Logo, $F\left({ }_{0} W_{j}^{\prime \prime}\right) \in[0, k]$, pois $F(W)=0$ e $F\left({ }_{0} W_{j^{\prime}}^{\prime}\right) \in[0, k]$. Como queríamos.

Agora mostraremos que se $D$ é transitivo então $R_{k}^{+}$é uma $G$-congruência. Suponha que $u R_{k}^{+} v$. Provaremos que para todo $g \in G$, temos ${ }^{g} u R_{k}^{+}{ }^{g} v$. Sejam $g \in G$ e

$$
W^{\prime}=\left(u_{0}, \epsilon_{1}, \ldots, \epsilon_{n}, u_{n}\right)
$$

um passeio de $R_{k}^{+}[u, v]$. Como $g$ preserva arestas temos um passeio

$$
{ }^{g} W=\left({ }^{g} u_{0}, \epsilon{ }_{1},{ }^{g} u_{1}, \ldots, \epsilon{ }_{n},{ }^{g} u_{n}\right)
$$

e claramente ${ }^{g} W \in R_{k}^{+}\left[{ }^{g} u,{ }^{g} v\right]$. De forma análoga, prova-se que se ${ }^{g} u R_{k}^{+}{ }^{g} v$, então $u R_{k}^{+} v$. Portanto $R_{k}^{+}$é uma $G$-congruência. Analogamente prova-se para $R_{k}^{-}$.

Denotaremos por $R_{k}^{+}(v)$ (resp. $R_{k}^{-}(v)$ ) a classe de equivalência do vértice $v$. Observe, se $u R_{k}^{+} v$ então $u R_{k+1} v$, de forma análoga para o caso $R_{k}^{-}$. Deste modo, temos que as sequências $\left(R_{k}^{+}\right)_{k \in \mathbb{Z}^{+}}$ e $\left(R_{k}^{-}\right)_{k \in \mathbb{Z}^{+}}$são ascendentes: para todo $k$ temos $R_{k}^{+} \subseteq R_{k+1}^{+}$e $R_{k}^{-} \subseteq R_{k+1}^{-}$. Pela proposição acima temos que, se $D$ é transitivo, então as classes de $R_{k}^{+}$(e similarmente $R_{k}^{-}$) formam um sistema de imprimitividade para $A u t(D)$. Dessa forma, em um dígrafo conexo contendo laços em todos os seus vértices as relações $R_{k}^{+}$e $R_{k}^{-}$são universais. Além disso, suas respectivas uniões

$$
R^{+}=\bigcup_{k \in \mathbb{Z}^{+}} R_{k}^{+} \quad \text { e } \quad R^{-}=\bigcup_{k \in \mathbb{Z}^{+}} R_{k}^{-}
$$


são também relações de equivalência, e se $D$ é transitivo, então suas classes formam um sistema de imprimitividade para $\operatorname{Aut}(D)$

Enfatizamos que ao longo deste trabalho, $k$ é sempre um inteiro maior ou igual à 1 . E os dígrafos estudados tem grau de entrada e de saída pelos menos igual a 1.

Apresentamos nas seções seguintes alguns resultados provados em [11], tais resultados mostram como as propriedades das relações de alcance estão intimamente relacionadas com propriedades de dígrafos, tais como crescimento e ter propriedade $Z$. Primeiramente apresentamos algumas propriedades básicas destas relações. E, depois daremos uma condição suficiente para um dígrafo ter propriedade $Z$. Finalmente, apresentaremos o Teorema 3.21, o qual nos dá uma condição suficiente para um dígrafo ter crescimento exponencial.

\subsection{Algumas propriedades de $R_{k}^{+}$e $R_{k}^{-}$}

Sejam $D$ um dígrafo e $u, v \in V D$. Observe que todo passeio em $R_{1}^{+}[u, v]$ é um passeio alternado, isto é um passeio da forma

$$
\left(u_{0}, 1, u_{1},-1, u_{2}, 1, \ldots, u_{n-2}, 1, u_{n-1},-1, u_{n}\right) .
$$

Em geral, chamaremos de passeio k-alternado o passeio que é uma concatenação de passeios da forma

$$
\left(u_{0}, 1, u_{1}, 1, u_{2}, 1, \ldots, 1, u_{k},-1, \ldots,-1, u_{2 k-1},-1, u_{2 k}\right) .
$$

É claro que para $k \geq 2$, todo passeio $k$-alternado está em $R_{k}^{+}[u, v]$, mas nem todo passeio em $R_{k}^{+}[u, v]$ é $k$-alternado. Entretanto, o resultado a seguir mostra que se $u R_{k}^{+} v$ então existe um passeio $k$ alternado ligando $u$ a $v$. Os dois próximos resultados podem serem encontrados em [[13], Lema 3.1 e Proposição 3.2].

Lema 3.2. Sejam $D$ um dígrafo $k \geq 1$ um inteiro. Então, para quaisquer dois vértices $u, v \in V D$, temos que $u R_{k}^{+} v$ se, e somente se, existe um passeio $k$-alternado $W$ ligando u até $v$. Um resultado análogo vale para $R_{k}^{-}$.

Demonstração. Provaremos para o caso $R_{k}^{+}$. Sejam $u, v \in V D$. Suponha que existe um passeio $k$-alternado $W$ de $u$ a $v$. Então, $W \in R_{k}^{+}[u, v]$. 
Reciprocamente, suponha que $u R_{k}^{+} v$. Como o grau de entrada e saída é no mínimo 1 , existe um passeio direcionado de peso positivo ou negativo começando em qualquer vértice de $D$. Dado um passeio $W=\left(u_{0}, \epsilon_{1}, \ldots, \epsilon_{n}, u_{n}\right)$ em $R_{k}^{+}[u, v]$, obteremos um novo passeio $k$ alternado, inserindo um passeio de comprimento apropriado em cada vértice $u_{i}$ tal que $\epsilon_{i} \neq \epsilon_{i+1}$ e $F\left({ }_{0} W_{i}\right) \notin\{0, k\}$. Seja $i \in\{1, \ldots, n\}$ o menor inteiro tal que $\epsilon_{i} \neq \epsilon_{i+1}$ e $F\left({ }_{0} W_{i}\right) \notin\{0, k\}$. Digamos $F\left({ }_{0} W_{i}\right)=l$. Seja $j<i$ o menor inteiro tal que $\epsilon_{j} \neq \epsilon_{j+1}$. Temos então dois casos.

- Caso 1. Se $F\left({ }_{0} W_{j}\right)=0$. Neste caso, $\epsilon_{i}=1$ e $\epsilon_{i+1}=-1$, então substituímos $W$ pelo passeio

$$
W^{\prime}={ }_{0} W_{i} \cdot\left(x_{0}, 1, x_{1}, 1, \ldots, 1, x_{k-l},-1, x_{(k-l)-1},-1, \ldots,-1, x_{0}\right) \cdot{ }_{i} W_{n} .
$$

Logo, em $W^{\prime}$ temos $F\left({ }_{0} W_{i+k-l}^{\prime}\right)=k, \mathrm{e}$

$$
\left(x_{k-l},-1, x_{(k-l)-1},-1, \ldots,-1, x_{0},-1, u_{i+1}, \ldots,-1, u_{i^{\prime}}\right)
$$

é um caminho direcionado de comprimento menor ou igual a $k$, onde $i^{\prime}>i$ é o próximo índice satisfazendo $\epsilon_{i^{\prime}} \neq \epsilon_{i^{\prime}+1}$ e $F\left({ }_{0} W_{i^{\prime}}\right) \notin\{0, k\}$.

- Caso 2. Se $F\left({ }_{0} W_{j}\right)=k$. Neste caso, temos $\epsilon_{i}=-1$ e $\epsilon_{i+1}=1$. Similarmente, substituímos $W$ pelo passeio

$$
W^{\prime}={ }_{0} W_{i} \cdot\left(x_{0},-1, x_{1},-1, \ldots,-1, x_{k-l}, 1, x_{(k-l)-1}, 1, \ldots, 1, x_{0}\right) \cdot{ }_{i} W_{n} .
$$

Assim em $W^{\prime}$ temos $F\left({ }_{0} W_{i+(k-l)}^{\prime}\right)=0$ e

$$
\left(x_{k-l}, 1, x_{(k-l)-1}, 1, \ldots, 1, x_{0}, 1, u_{i+1}, \ldots, 1, u_{i^{\prime}}\right)
$$

é um caminho direcionado de comprimento menor ou igual a $k$, onde $i^{\prime}>i$ é o próximo índice satisfazendo $\epsilon_{i^{\prime}} \neq \epsilon_{i^{\prime}+1}$ e $F\left({ }_{0} W_{i^{\prime}}\right) \notin\{0, k\}$.

Repetindo o processo para $W^{\prime}$ e após um número finito de passos, obtemos o passeio desejado. 
Corolário 3.3. Seja $D=\operatorname{Cay}(G, S)$ um dígrafo de Cayley de um grupo $G$ com respeito ao conjunto $S$ de geradores. Então para qualquer inteiro $k \geq 1$ e qualquer $g \in G$ temos que

$$
R_{k}^{+}(g)=g R_{k}^{+}(e)=g\left\langle S^{k} . S^{-k}\right\rangle \text { e } R_{k}^{-}(g)=g R_{k}^{-}(e)=g\left\langle S^{-k} . S^{k}\right\rangle
$$

onde e é o elemento identidade de $G$.

Demonstração. Seja $g \in G$. Primeiramente mostraremos que $g R_{k}^{+}(e) \subseteq R_{k}^{+}(g)$. Sejam $u \in R_{k}^{+}(e)$ e $W$ um passeio de $R_{k}^{+}[u, e]$. Como $G$ age sobre $D$ por multiplicação à esquerda, temos um passeio ${ }^{g} W$ em $R_{k}^{+}[g u, g]$. Assim $g u \in R_{k}^{+}(g)$. De forma similar obtemos $R_{k}^{+}(g) \subseteq g R_{k}^{+}(e)$. Logo, $R_{k}^{+}(g)=g R_{k}^{+}(e)$. Analogamente, temos $R_{k}^{-}(g)=g R_{k}^{-}(e)$.

Agora, provaremos que $R_{k}^{+}(e)=\left\langle S^{k} . S^{-k}\right\rangle$. Seja $v \in R_{k}^{+}(e)$. Pelo Lema 3.2 existe um passeio $W \in R_{k}^{+}[e, v]$ que é um passeio $k$-alternado, digamos $W=W_{1} W_{2} \ldots W_{n}$, onde

$$
W_{i}=\left(w_{0}^{i}, 1, w_{1}^{i}, \ldots, 1, w_{k}^{i},-1, w_{k+1}^{i}, \ldots,-1, w_{2 k}^{i}\right)
$$

Tome o subpasseio $W_{1}$. Como por hipótese $D$ é um dígrafo de Cayley, temos

$$
W_{1}=\left(e, 1, s_{1}, 1, s_{1} s_{2}, 1, \ldots, 1, s_{1} \ldots s_{k},-1, s_{1} \ldots s_{k} s_{k+1}^{-1},-1, \ldots,-1, s_{1} \ldots s_{k} s_{k+1}^{-1} \ldots s_{2 k}^{-1}\right)
$$

com $s_{i} \in S$ para todo $i$. Portanto o vértice final de $W_{1}$ é da forma

$$
w_{2 k}^{1}=s_{1} \ldots s_{k} s_{k+1}^{-1} \ldots s_{2 k}^{-1} \in\left\langle S^{k}, S^{k}\right\rangle .
$$

Repetindo, o mesmo argumento para cada $W_{i}$, concluímos que $v \in\left\langle S^{k} . S^{-k}\right\rangle$. Similarmente obtemos $\left\langle S^{k}, S^{-k}\right\rangle \subseteq R_{k}^{+}(e)$. De forma análoga prova-se que $g R_{k}^{-}(e)=g\left\langle S^{-k} \cdot S^{k}\right\rangle$. Com isto provamos o resultado.

A seguir consideraremos as relações $R_{k}^{+}$e $R_{k}^{-}$sob diferentes aspectos. Primeiro, daremos algumas condições necessárias e suficientes para as sequências $\left(R_{k}^{+}\right)_{k \in \mathbb{Z}^{+}} \mathrm{e}\left(R_{k}^{-}\right)_{k \in \mathbb{Z}^{+}}$estabilizarem isto é, existem $i, j$ tais que $R^{+}=R_{i}^{+}$e $R^{-}=R_{j}^{-}$. Depois estudaremos propriedades das classes de equivalências de cada relação $R_{k}^{+}$e $R_{k}^{-}$. 


\subsubsection{As sequências $\left(R_{k}^{+}\right)_{k \in \mathbb{Z}^{+}}$e $\left(R_{k}^{-}\right)_{k \in \mathbb{Z}^{+}}$}

Como já foi observado no início deste capítulo, em um dígrafo conexo contendo laços em todos os seus vértices as relações $R_{k}^{+}$e $R_{k}^{-}$são universais.

Agora daremos dois exemplos para o caso em que todos os vértices do dígrafo $D$ estão contidos em ciclos direcionados de comprimento 2. No primeiro exemplo $R_{2}^{+}$é universal, enquanto no segundo exemplo $R_{2}^{+}$tem duas classes de equivalências.

Exemplo 3.4. Seja $D=\operatorname{Cay}\left(S_{3}, S\right)$, onde $S_{3}=\left\langle r, t \mid r^{3}=t^{2}=1, r t r=t\right\rangle$ e $S=\{r, t\}$. Então temos que

$$
\begin{gathered}
R_{1}^{+}(e)=\{e, r t\} \\
R_{2}^{+}(e)=S_{3},
\end{gathered}
$$

portanto $R_{2}^{+}$é universal. Além disso, observe na Figura 2.3 que cada vértice de D está contido em um passeio direcionado de comprimento 2 , e existem ciclos em $D$ de comprimento ímpar.

Exemplo 3.5. Seja $D=C a y\left(D_{4}, S\right)$, onde $D_{4}=\left\langle r, t \mid r^{4}=t^{2}=1, r t r=t\right\rangle$ e $S=\{r, t\}$. Então temos que

$$
\begin{aligned}
& R_{1}^{+}(e)=\{e, r t\} \text { e } R_{2}^{+}(e)=\left\{e, r^{2}, r t, r^{3} t\right\} \\
& R_{1}^{+}(t)=\left\{t, r^{3}\right\} \text { e } R_{2}^{+}(t)=\left\{t, r^{3}, r, r^{2} t\right\} \\
& R_{3}^{+}(e)=R_{2}^{+}(e) \text { e } R_{3}^{+}(t)=R_{2}^{+}(t) .
\end{aligned}
$$

Portanto $R_{2}^{+}$tem duas classes de equivalências. Além disso, observe na Figura 2.4 que cada vértice de D está contido em um passeio direcionado de comprimento 2 , e não existem ciclos em $D$ de comprimento impar.

Em geral, temos.

Proposição 3.6. Seja D um dígrafo conexo, em que cada vértice está contido em um passeio direcionado fechado de comprimento 2. Então $R^{+}=R_{2}^{+}=R^{-}=R_{2}^{-}$. Além disso,

(i) Se existe um passeio fechado de comprimento ímpar em $D$, então $R_{2}^{+}$(e $R_{2}^{-}$) é universal, $e$

(ii) Se não existe tal passeio em $D$ então $R_{2}^{+}$( e $R_{2}^{-}$) tem duas classes de equivalências. 
Demonstração. Primeiro mostraremos que dados quaisquer $u$ e $v \in V D$, um passeio arbitrário $W=\left(v_{0}, \epsilon_{1}, v_{1}, \ldots, \epsilon_{n}, v_{n}\right)$ de $u$ a $v$ pode ser modificado de modo que obtemos um novo passeio $W^{\prime}$ de $u$ a $v$ com as seguintes propriedades:

$$
\left\{\begin{array}{l}
F\left(W^{\prime}\right)=0 \text { se }|W| \text { é par } \\
F\left(W^{\prime}\right)=1 \text { se }|W| \text { é ímpar } \\
F\left({ }_{0} W_{j}^{\prime}\right) \in[0,2], \text { para todo } 0 \leq j \leq\left|W^{\prime}\right|
\end{array}\right.
$$

Começaremos com o passeio trivial, ou seja $W=(u)$. Redefiniremos $W^{\prime}$ ao atingirmos o j-ésimo vértice de $W$, supondo as seguintes possibilidades: $j$ é par e $F\left({ }_{0} W_{j}^{\prime}\right)$ é igual a 0 ou 2 (podemos também supor que $j$ é ímpar tal que $F\left({ }_{0} W_{j}^{\prime}\right)=1$ e redefinir $W^{\prime}$ de forma análoga ao caso quando $j$ é par). Por hipótese de construção temos que o peso de $W^{\prime}$ é ou 0 , ou 1 ou 2 . Então, a partir de $v_{j}$ faremos as modificações em $W^{\prime}$.

\section{Caso 1: Se}

(1.1) $F\left(W^{\prime}\right)=0$ e $\epsilon_{j+1}=1$, por hipótese de construção, segue que $\left|W^{\prime}\right|$ é par;

(1.2) $F\left(W^{\prime}\right)=2$ e $\epsilon_{j+1}=-1$, por hipótese de construção, temos que $\left|W^{\prime}\right|$ é par;

(1.3) $F\left(W^{\prime}\right)=1$ e $\epsilon_{j+1}$ arbitrário, por hipótese de construção, segue que $\left|W^{\prime}\right|$ é ímpar.

Então, adicionamos ao passeio $W^{\prime}$ a aresta de indicador $\epsilon_{j+1}$, isto é, $W^{\prime}=W^{\prime}\left(v_{j}, \epsilon_{j+1}, v_{j+1}\right)$. Note que em (1.1) e (1.2), obtemos após as modificações $F\left(W^{\prime}\right)=1$, agora em (1.3) temos $F\left(W^{\prime}\right)$ é igual à 0 ou 2. E se $W=W^{\prime}$, a condição 3.1 se verifica, visto que em (1.1) e (1.2) $\left|W^{\prime}\right|$ é ímpar e em (1.3) $\left|W^{\prime}\right|$ é par, após as modificações.

Caso 2: Se $F\left(W^{\prime}\right)=0$ e $\epsilon_{j+1}=-1$, ( veja que este caso, pode ser o passo 1, ou seja, quando $\left.\epsilon_{1}=-1\right)$. Por hipótese, qualquer vértice em $D$ está contido em um ciclo direcionado de comprimento 2. Logo, existe $w \in D$ tal que $\left(v_{j}, w\right)$ e $\left(w, v_{j}\right) \in D$. Neste caso, redefinimos $W^{\prime}=W^{\prime}\left(v_{j}, 1, w, 1, v_{j}, \epsilon_{j+1}, v_{j+1}\right)$. Observe que, após as modificações, obtemos $F\left(W^{\prime}\right)=1$ e $\left|W^{\prime}\right|$ é ímpar, uma vez que antes das mudanças ocorridas em $W$, tínhamos que $\left|W^{\prime}\right|$ era par, por hipótese de construção. 
Caso 3: Se $F\left(W^{\prime}\right)=2$ e $\epsilon_{j+1}=1$. Então redefinimos $W^{\prime}=W^{\prime}\left(v_{j},-1, w,-1, v_{j}, \epsilon_{j+1}, v_{j+1}\right)$, onde $w$ é considerado semelhante ao caso 2. Veja que, após as modificações, obtemos $F\left(W^{\prime}\right)=$ 1 e $\left|W^{\prime}\right|$ é ímpar.

Observe que, em cada caso foram construído subpasseios com peso ou 0 ou 1 ou 2. Continuando dessa maneira terminaremos com um passeio $W^{\prime}$ de $u$ à $v$, com a propriedade que queremos, exceto a possibilidade $F\left(W^{\prime}\right)=2$. Se isto ocorrer, então redefinimos $W^{\prime}$, agora como sendo $W^{\prime}=W^{\prime}(v,-1, w,-1)$ para um apropriado vértice $w$. Para provarmos os itens (i) e (ii), precisamos das seguintes afirmações:

Afirmação 1: Sejam $u, v \in V D$ então $u R_{2}^{+} v$ se, e somente se, existe um passeio de comprimento par ligando $u \grave{a} v$.

De fato, seja $u, v \in V D$ e $W$ o passeio ligando $u$ à $v$, tal que $|W|$ é par então pela construção feita acima, podemos obter um passeio $W^{\prime}$ de $u$ à $v$ tais que $F\left(W^{\prime}\right)=0$ e $F\left({ }_{i} W_{j}^{\prime}\right) \in[0,2]$, para todo $0 \leq j \leq\left|W^{\prime}\right|$, portanto $u R_{2}^{+} v$.

Reciprocamente, suponha que $u R_{2}^{+} v$. Seja $W$ um passeio de $R_{2}^{+}[u, v]$, isto implica que $F(W)=0$, ou seja, temos o mesmo número de arestas com indicadores 1 quanto de arestas com indicadores -1, portanto $|W|$ é par. Com isto provamos a afirmação.

Seja $(u, v) \in E D$. Pela Afirmação 1, existem as seguintes classes de equivalências:

$$
\begin{aligned}
& R_{2}^{+}(u)=\left\{w_{1} \in V D \mid \text { existe um passeio de comprimento par ligando } u \text { à } w_{1}\right\} \\
& R_{2}^{+}(v)=\left\{w_{2} \in V D \mid \text { existe um passeio de comprimento par ligando } v \text { à } w_{2}\right\}
\end{aligned}
$$

Agora, se mostrarmos que $u R_{2}^{+} v$ então pela transitividade de $R_{2}^{+}$, obteremos que $R_{2}^{+}$é universal. Como veremos a seguir.

Afirmação 2: Se existe um passeio fechado $C$ em $D$ de comprimento ímpar, então $R_{2}^{+}$é universal, ou seja $R_{2}^{+}$tem somente uma classe de equivalência. 


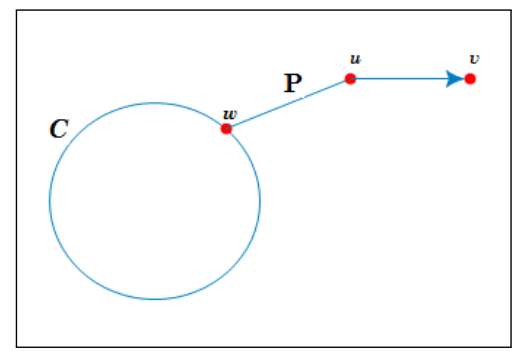

Figura 3.1: passeio

De fato, seja $C$ um passeio fechado (Figura 3.1) de comprimento ímpar. Por hipótese $D$ é conexo, logo existe em $D$ um passeio $P$ ligando $u$ à um vértice $w$ de $C$. Temos então que o comprimento de $P$ ou é par ou é ímpar. Tome o passeio $W^{\prime \prime}=P C P^{-1}(u, 1, v)$. Note que $W^{\prime \prime}$ é um passeio de $u$ à $v$ e $\left|W^{\prime \prime}\right|$ é par, tanto para o caso em que $|P|$ é par quanto para o caso em que $|P|$ é ímpar. E segue da Afirmação 1 que $u R_{2}^{+} v$. Pela transitividade da relação $R_{2}^{+}$, obtemos que $R_{2}^{+}$é universal.

Finalmente, mostraremos que $R_{2}^{+}=R^{+}$. Por definição sabemos que $R_{2}^{+} \subseteq R^{+}$. Sejam $u, v \in V D$ tal que $u R_{2}^{+} v$. Observe que quando construímos o passeio $W^{\prime}$, em nenhum momento usamos a relação $R_{2}^{+}$, logo podemos afirmar que $u R^{+} v$ se, e somente se, existe um passeio $W$ de comprimento par ligando $u$ à $v$. Portanto, $u R_{2}^{+} v$ como queríamos, daí $R_{2}^{+}=R^{+}$. $\mathrm{A}$ prova para $R_{2}^{-}$é feita de modo similar. Como as condições para dois vértices estarem $R_{2}^{+}$ relacionados coincide com a condição de estarem $R_{2}^{-}$-relacionados, segue que $R_{2}^{+}=R_{2}^{-}$. Portanto $R^{+}=R_{2}^{+}=R^{-}=R_{2}^{-}$.

Com argumentos similares ao que foi feito na Proposição 3.6, é possível provar para um dígrafo $D$, onde cada vértice de $D$ está contido em um ciclo direcionado de comprimento $k$, que $R^{+}=R_{k}^{+}=R_{k}^{-}=R^{-}$. Uma prova usando métodos diferentes pode ser encontrada em [18]. Temos também que uma, ou ambas, as sequências $\left(R_{k}^{+}\right)_{k \in \mathbb{Z}^{+}}$e $\left(R_{k}^{-}\right)_{k \in \mathbb{Z}^{+}}$podem ser finitas, embora o dígrafo em questão não tenha ciclos direcionado. Contudo, os resultados que serão apresentados na seção 3.2 mostram que o fato das sequências $\left(R_{k}^{+}\right)_{k \in \mathbb{Z}^{+}}$e $\left(R_{k}^{-}\right)_{k \in \mathbb{Z}^{+}}$serem finitas ou infinitas tem grande importância para estudar as propriedades de dígrafos tais como crescimento e ter propriedade $Z$. Daremos, agora três condições necessárias e suficientes para as sequências $\left(R_{k}^{+}\right)_{k \in \mathbb{Z}^{+}}$e $\left(R_{k}^{-}\right)_{k \in \mathbb{Z}^{+}}$serem finitas. 
Proposição 3.7. Seja $D$ um dígrafo. Então $R^{+}=R_{k}^{+}$para algum $k$ se, e somente se, $R_{k+1}^{+}=R_{k}^{+}$. Uma afirmação análoga vale para $R^{-}$.

Demonstração. Suponha $R^{+}=R_{k}^{+}$, para algum $k$. Note que $R_{k}^{+} \subseteq R_{k+1}^{+} \subseteq R^{+}=R_{k}^{+}$, portanto $R_{k}^{+}=R_{k+1}^{+}$.

Reciprocamente, suponha que existe um inteiro positivo $k$ tal que $R_{k+1}^{+}=R_{k}^{+}$. Mostraremos que $R_{k+1}^{+}=R_{k}^{+}=R_{k+2}^{+}$. Por definição, $R_{k+1}^{+} \subseteq R_{k+2}^{+}$, logo resta mostrar que $R_{k+2}^{+} \subseteq R_{k+1}^{+}$. E consequentemente por indução teremos $R^{+}=R_{k}^{+}$. Sejam $u, v \in V D$ tal que $u R_{k+2}^{+} v$ e seja $W=\left(v_{0}, \epsilon_{1}, v_{1}, \ldots, \epsilon_{n}, v_{n}\right)$ um passeio em $R_{k+2}^{+}[u, v]$. Tome o menor inteiro $j$, tal que $F\left({ }_{0} W_{j}\right)=k+2$, onde $0<j<n$. Se tal inteiro não existe, então $R_{k+2}^{+} \subseteq R_{k+1}^{+}$, logo não há o que provar. Assuma que $j$ existe. Seja $0<j_{1}<j$ o maior inteiro tal que $F\left({ }_{0} W_{j_{1}}\right)=1$ e seja $j<j_{2}<n$ o menor inteiro tal que $F\left(j_{2} W_{n}\right)=-1$.

Afirmação: O passeio ${ }_{j_{1}} W_{j_{2}} \in R_{k+1}^{+}\left[v_{j_{1}}, v_{j_{2}}\right]$.

De fato, de acordo com a escolha de $v_{j_{1}}$ e $v_{j_{2}}$, temos que:

$$
F\left({ }_{j_{1}} W_{j_{2}}\right)=F(W)-F\left({ }_{0} W_{j_{1}}\right)-F\left({ }_{j_{2}} W_{n}\right)=0-1+1=0
$$

e $F\left({ }_{j_{1}} W_{t}\right) \in[0, k+1]$ para todo $j_{1} \leq t \leq j_{2}$. Pois, se $j_{1} \leq t \leq j$ então $F\left(j_{1} W_{t}\right) \in[0, k+1]$, caso contrário irá contradizer a minimalidade de $j$. E se $j \leq t \leq j_{2}$, então $F\left(j_{1} W_{t}\right) \in[0, k+1]$, pois senão, poderíamos ter que $F\left(j_{1} W_{t}\right)=k+2$, daí $F\left({ }_{0} W_{t}\right)=k+3$, absurdo. Isto prova afirmação. Assim pela Afirmação, temos que $v_{j_{1}} R_{k+1}^{+} v_{j_{2}}$, entretanto por hipótese $R_{k+1}^{+}=R_{k}^{+}$, $\operatorname{logo} v_{j_{1}} R_{k}^{+} v_{j_{2}}$, isto é, existe um passeio $W^{\prime} \in R_{k}^{+}\left[v_{j_{1}}, v_{j_{2}}\right]$. Agora, considere o passeio

$$
W^{\prime \prime}={ }_{0} W_{j_{1}} \cdot W^{\prime} \cdot j_{2} W_{n}
$$

Se ainda há algum $0<j^{\prime}<\left|W^{\prime \prime}\right|$ tal que $F\left({ }_{0} W_{j^{\prime}}^{\prime \prime}\right)=k+2$, repita o processo acima. Continuando dessa maneira, ou seja eliminando os "picos", onde $F\left({ }_{0} W_{j^{\prime}}^{\prime \prime}\right)=k+2$, obteremos um passeio $W^{*}$ de $R_{k+1}^{+}[u, v]$, de fato, basta observar que são necessários somente um número finito de repetições do processo, uma vez que ao realizarmos cada etapa, diminuímos nosso campo onde ocorrem os "picos", por exemplo na etapa 2 , temos que $j_{2}<j^{\prime}<n$, porque qualquer subpasseio de ${ }_{0} W_{j_{1}} \cdot W^{\prime}$ tem peso no máximo igual à $k+1$, pois por suposição temos que $F\left({ }_{0} W_{j_{1}}\right)=1$ e o peso de 
qualquer subpasseio iniciando em $v_{j_{1}}$ e que pertença à $W^{\prime}$ atinge no máximo $k+1$. Portanto $R_{k+2}^{+} \subseteq R_{k+1}^{+}$, como queríamos.

De forma análoga, prova-se para o caso $R^{-}$.

Uma consequência imediata da proposição acima é que estas sequências são infinitas se, e somente se, elas são estritamente crescentes.

Além disso, conforme mostrado na Proposição 3.7, $R^{+}=R_{k}^{+}$sempre que $R_{k}^{+}=R_{k+1}^{+}$. Neste caso, o menor inteiro $k$ tal que $R_{k}^{+}=R_{k+1}^{+}$é chamado de expoente $\exp ^{+}(D)$ de $D$. Se $R^{+} \neq R_{k}^{+}$, para todo $k$ então dizemos que $\exp ^{+}(D)=\infty$. De forma similar, definimos o expoente $\exp ^{-}(D)$.

Proposição 3.8. Seja $D$ um dígrafo. Então para cada inteiro $k \geq 1$, as seguintes afirmações são equivalentes:

(i) $R^{+}=R_{k}^{+}$;

(ii) $R_{k+1}^{+}=R_{k}^{+}$;

(iii) $R_{1}^{+} \subseteq R_{k}^{-}$;

(iv) $R^{+} \subseteq R_{k}^{-}$.

Trocando os sinais $+e-$, obtemos um resultado análogo.

Demonstração. Da Proposição 3.7 segue que (i) e (ii) são equivalentes. Agora mostraremos que (i) implica (iv). Seja $u, v \in V D$ tal que $u R^{+} v$, e por hipótese temos $u R_{k}^{+} v$. Seja $W$ um passeio de $R_{k}^{+}[u, v]$. Escolheremos dois vértices $u^{*}$ e $v^{*}$ juntamente com os passeios direcionados $W^{u}$ e $W^{v}$ de comprimento $k$, ligando $u^{*}$ à $u$ e $v^{*}$ à $v$, respectivamente. Consideremos o passeio $W^{\prime}=W^{u} \cdot W \cdot\left(W^{v}\right)^{-1}$.

Afirmação: O passeio $W^{\prime} \in R_{2 k}^{+}\left[u^{*}, v^{*}\right]$.

De fato, note que

$$
F\left(W^{\prime}\right)=F\left(W^{u}\right)+F(W)+F\left(\left(W^{v}\right)^{-1}\right)=k+0-k=0,
$$

agora falta mostrar que para todo $0 \leq j \leq\left|W^{\prime}\right|$ o peso do subpasseio ${ }_{0} W_{j}^{\prime}$, pertence ao intervalo $[0,2 k]$. Dividiremos em três possíveis casos: 
- Se $0 \leq j \leq\left|W^{u}\right|$, então $F\left({ }_{0} W_{j}^{\prime}\right)=F\left({ }_{0} W_{j}^{u}\right) \in[0, k]$, pois $W^{u}$ é um passeio direcionado de peso $k$.

- Se $\left|W^{u}\right| \leq j \leq\left|W^{u}\right|+|W|$, então

$$
F\left({ }_{0} W_{j}^{\prime}\right)=F\left(W^{u}\right)+F\left(\left|W^{u}\right| W_{j}^{\prime}\right)=k+F\left(\left|W^{u}\right| W_{j}^{\prime}\right)
$$

mas $\left(\left|W^{u}\right| W_{j}^{\prime}\right)$ é um subpasseio de $W \in R_{k}^{+}[u, v]$, logo $F\left(\left|W^{u}\right| W_{j}^{\prime}\right) \in[0, k]$, e portanto $F\left({ }_{0} W_{j}^{\prime}\right) \in[k, 2 k]$.

- Se $\left|W^{u}\right|+|W| \leq j \leq\left|W^{\prime}\right|$, então

$$
F\left({ }_{0} W_{j}\right)=F\left(W^{u}\right)+F(W)+F\left(|W|+\left|W^{u}\right| W_{j}^{\prime}\right)=k+F\left(|W|+\left|W^{u}\right| W_{j}^{\prime}\right) .
$$

Observe que $\left(|W|+\left|W^{u}\right| W_{j}^{\prime}\right) \quad$ é um subpasseio de $\quad\left(W^{v}\right)^{-1}$, e isto implica que $F\left(|W|+\left|W^{u}\right| W_{j}^{\prime}\right) \in[-k, 0]$, portanto $F\left({ }_{0} W_{j}^{\prime}\right) \in[0, k]$. Como queríamos. Agora, usando a hipótese de que $R^{+}=R_{k}^{+}$e a Afirmação, temos que existe um passeio $W^{*} \in R_{k}^{+}\left[u^{*}, v^{*}\right]$. Logo, o passeio $W^{\prime \prime}=\left(W^{u}\right)^{-1} \cdot W^{*} \cdot W^{v}$ pertence a $R_{k}^{-}[u, v]$, isto é provado de maneira análoga ao que foi feito na Afirmação. Portanto, de $W^{\prime \prime}=\left(W^{u}\right)^{-1} . W \cdot W^{v} \in R_{k}^{-}[u, v]$ segue que $u R_{k}^{-} v$, ou seja, $R^{+} \subseteq R_{k}^{-}$. Como $R_{1}^{+} \subseteq R^{+}$e $R^{+} \subseteq R_{k}^{-}$, por hipótese, então segue que $R_{1}^{+} \subseteq R_{k}^{-}$. Logo (iv) implica (iii).

Resta provarmos apenas que (iii) implica (ii). Consideremos $u R_{k+1}^{+} v$. Sejam

$$
W=\left(v_{0}, \epsilon_{1}, v_{1}, \ldots, \epsilon_{n}, v_{n}\right)
$$

um passeio de $R_{k+1}^{+}[u, v]$ e $0<j<n$ o menor inteiro tal que $F\left({ }_{0} W_{j}\right)=k+1$. Então

$$
F\left({ }_{0} W_{j-1}\right)=k=F\left({ }_{0} W_{j+1}\right)
$$

De fato, por suposição, $F\left({ }_{0} W_{j}\right)=k+1$ e, ou $\epsilon_{j}=1$ ou $\epsilon_{j}=-1$. De $F\left({ }_{0} W_{j-1}\right)=F\left({ }_{0} W_{j}\right)-\epsilon_{j}$, obtemos que $F\left({ }_{0} W_{j-1}\right)=k+1-\epsilon_{j}$, e portanto, $\epsilon_{j}=1$, caso contrário teríamos que $F\left({ }_{0} W_{j-1}\right)=k+2$, absurdo. Similarmente, obtemos que $\epsilon_{j+1}=-1$. Logo, $F\left({ }_{0} W_{j-1}\right)=k=F\left({ }_{0} W_{j+1}\right)$, e isto implica que o passeio $W^{\oplus}=\left(v_{j-1}, 1, v_{j},-1, v_{j+1}\right)$ pertence 
à $R_{1}^{+}\left[v_{j-1}, v_{j+1}\right]$. Mas, por hipótese, $R_{1}^{+} \subseteq R_{k}^{-}$, logo existe um passeio $W^{\prime} \in R_{k}^{-}\left[v_{j-1}, v_{j+1}\right]$. Defina o passeio

$$
W^{\prime \prime}={ }_{0} W_{j-1} \cdot W^{\prime} \cdot{ }_{j+1} W_{n}
$$

Se ainda há algum $0<j^{\prime}<\left|W^{\prime \prime}\right|$ tal que $F\left({ }_{0} W_{j}^{\prime \prime}\right)=k+1$, repita o processo anterior. Continuando, dessa maneira, obteremos um passeio pertencente à $R_{k}^{+}[u, v]$, como queríamos. Se $j^{\prime}$ não existe então o resultado foi provado.

Corolário 3.9. Seja $D$ um dígrafo. Suponha que $R_{k}^{+}=R_{k}^{-}$para algum inteiro $k \geq 1$. Então

$$
R^{+}=R_{k}^{+}=R_{k}^{-}=R^{-}
$$

Demonstração. Como por hipótese, $R_{k}^{+}=R_{k}^{-}$, segue que $R_{1}^{+} \subseteq R_{k}^{-}$. Da Proposição 3.8 acima temos que $R_{k+1}^{+}=R_{k}^{+}$. Logo pela Proposição 3.8, obtemos que $R^{+}=R_{k}^{+}$. De forma análoga, prova-se que $R^{-}=R_{k}^{-}$, e como por hipótese $R_{k}^{+}=R^{+}$, segue que $R^{+}=R_{k}^{+}=R_{k}^{-}=R^{-}$.

Corolário 3.10. Seja D um dígrafo. Então as seguintes afirmaçôes são equivalentes:

(i) Ambos os expoentes exp ${ }^{+}(D)$ e exp $\exp ^{-}(D)$ são finitos.

(ii) Existe algum inteiro $k \geq 1$ tal que $R^{+}=R_{k}^{+}=R_{k}^{-}=R^{-}$com $R_{k}^{+} \neq R_{k-1}^{+}, R_{k}^{-} \neq R_{k-1}^{-}$e $R_{k+1}^{+} \neq R_{k-1}^{-}$sempre que $k \geq 2$.

Demonstração. Suponha que ambos os expoentes são finitos, isto é existem inteiros mínimos $i, j \geq 1$ tais que $R^{+}=R_{i}^{+}$e $R_{j}^{-}=R^{-}$. Tome $k=\max (i, j)$. Então $R^{+}=R_{k}^{+}$e $R_{k}^{-}=R^{-}$. Isto implica, pela Proposição 3.8, que $R^{+} \subseteq R_{k}^{-}=R^{-}$e $R^{-} \subseteq R_{k}^{+}=R^{+}$. Assim,

$$
R^{+}=R_{k}^{+}=R_{k}^{-}=R^{-}
$$

Suponha agora $k \geq 2$, e além disso, suponha sem perda de generalidade que $R_{k}^{+} \neq R_{k-1}^{+}$e $R_{k}^{-}=R_{k-1}^{-}$. Então

$$
R^{+}=R_{k}^{+}=R_{k}^{-}=R_{k-1}^{-}
$$


Da Proposição 3.8, obtemos que $R_{k}^{+}=R_{k-1}^{+}$. Mas isto contradiz o fato que $R_{k}^{+} \neq R_{k-1}^{+}$. Portanto, $R_{k}^{+} \neq R_{k-1}^{+}$e $R_{k}^{-} \neq R_{k-1}^{-}$. Agora, como $R_{k}^{+} \neq R_{k-1}^{+}, k \geq 2$, segue do Corolário que $R_{k-1}^{-} \neq R_{k-1}^{+}$.

Reciprocamente, suponha que existe um inteiro $k \geq 1$ tal que $R^{+}=R_{k}^{+}=R_{k}^{-}=R^{-}$com $R_{k}^{+} \neq R_{k-1}^{+}, R_{k}^{-} \neq R_{k-1}^{-}$e $R_{k+1}^{+} \neq R_{k-1}^{-}$sempre que $k \geq 2$. Isto significa que

$$
\exp ^{+}(D)=\exp ^{-}(D)=k
$$

e portanto as sequências $\left(R_{k}^{+}\right)_{k \in \mathbb{Z}^{+}}$e $\left(R_{k}^{-}\right)_{k \in \mathbb{Z}^{+}}$são ambas finitas.

Em outras palavras, o corolário anterior mostra que ambas as sequências $\left(R_{k}^{+}\right)_{k \in \mathbb{Z}^{+}} \mathrm{e}\left(R_{k}^{-}\right)_{k \in \mathbb{Z}^{+}}$ são finitas se, e somente se, ambos expoentes são finitos e iguais.

Para finalizarmos esta subseção daremos dois exemplos: o primeiro com expoente $e x p^{+}$infinito e o segundo com $\exp ^{+}$finito.

Exemplo 3.11. A Figura 2.5 apresenta um pedaço de uma árvore regular com $d^{+}=1$ e $d^{-}=2$. Tome $v=v_{15}$. Note que

$$
\begin{aligned}
& \left\{v_{17}, v_{18}\right\} \subset R_{2}^{+}(v) \backslash R_{1}^{+}(v) \\
& \left\{v_{19}, v_{20}\right\} \subset R_{3}^{+}(v) \backslash R_{2}^{+}(v) \\
& \left\{v_{23}, v_{24}\right\} \subset R_{4}^{+}(v) \backslash R_{3}^{+}(v)
\end{aligned}
$$

Se continuarmos com este mesmo pensamento obtemos

$$
R_{1}^{+}(v) \subset R_{2}^{+}(v) \subset R_{3}^{+}(v) \subset \cdots R_{k}^{+}(v) \subset R_{k+1}^{+}(v) \subset \cdots
$$

Então, temos que as $R_{k}^{+}$-classes são estritamente crescentes.

Exemplo 3.12. A Figura 2.6 apresenta um dígrafo D com expoentes finitos, pois

$$
\exp ^{+}(D)=\exp ^{-}(D)=1
$$




\subsubsection{As classes de equivalências de $R_{k}^{+}$e $R_{k}^{-}$}

Seja $D$ um dígrafo. Lembre que estamos sempre considerando dígrafos com grau de saída e grau de entrada pelo menos igual à 1 . Neste caso, $D_{k}^{+}(v)$ e $D_{k}^{-}(v)$ são conjuntos não vazios para todo vértice $v \in V D$ e todo inteiro $k \geq 1$.

Os resultados a seguir tratam da relação entre as classes de equivalências de $R_{k}^{+}$e $R_{k}^{-}$e suas respectivas cardinalidades.

Proposição 3.13. Seja $D$ um dígrafo. Seja $v \in V D$ e $k \geq 1$ um inteiro. Então para cada $u \in D_{k}^{+}\left(R_{k}^{+}(v)\right)$ temos que $D_{k}^{+}\left(R_{k}^{+}(v)\right)=R_{k}^{-}(u)$. Trocando os sinais de $+e-$ obtemos um resultado análogo.

Demonstração. Por hipótese $u \in D_{k}^{+}\left(R_{k}^{+}(v)\right)$, logo existe um vértice $v^{\prime} \in R_{k}^{+}(v)$ e um passeio direcionado $W^{v^{\prime}}$ de comprimento $k$ ligando $v^{\prime}$ a $u$. Primeiramente mostraremos que $D_{k}^{+}\left(R_{k}^{+}(v)\right) \subseteq R_{k}^{-}(u)$. Seja $u^{\prime} \in D_{k}^{+}\left(R_{k}^{+}(v)\right)$. Assim, existe algum $v^{\prime \prime} \in R_{k}^{+}(v)$ e um passeio direcionado $W^{v^{\prime \prime}}$ de comprimento $k$ de $v^{\prime \prime}$ para $u^{\prime}$. Escolha os seguintes passeios $W^{\prime} \in R_{k}^{+}\left[v^{\prime}, v\right]$ e $W^{\prime \prime} \in R_{k}^{+}\left[v^{\prime \prime}, v\right]$. Defina o passeio $W=\left(W^{v^{\prime \prime}}\right)^{-1} \cdot W^{\prime \prime} \cdot\left(W^{\prime}\right)^{-1} \cdot W^{v^{\prime}}$.

Afirmação 1: O passeio $W \in R_{k}^{-}\left[u^{\prime}, u\right]$.

De fato, note que

$$
F(W)=F\left(\left(W^{v^{\prime \prime}}\right)^{-1}\right)+F\left(W^{\prime \prime}\right)+F\left(\left(W^{\prime}\right)^{-1}\right)+F\left(W^{v^{\prime}}\right)=-k+k=0 .
$$

Além disso, qualquer subpasseio ${ }_{0} W_{j} \in[-k, 0]$, para todo $0 \leq j \leq|W|$. Para ver que isto é válido, dividiremos em três casos dependendo de onde se encontra $j$.

- Se $0 \leq j \leq\left|\left(W^{v^{\prime \prime}}\right)^{-1}\right|=k$, então $F\left({ }_{0} W_{j}\right) \in[-k, 0]$;

- Se $k=\left|\left(W^{v^{\prime \prime}}\right)^{-1}\right| \leq j \leq\left|W^{\prime \prime}\right|+k$, então

$$
F\left({ }_{0} W_{j}\right)=F\left(\left(W^{v^{\prime \prime}}\right)^{-1}\right)+F\left({ }_{k} W_{j}\right)=-k+F\left({ }_{k} W_{j}\right),
$$

mas ${ }_{k} W_{j}$ é um subpasseio de $W^{\prime \prime}, \operatorname{logo}{ }_{k} W_{j} \in[0, k]$ e portanto $F\left({ }_{0} W_{j}\right) \in[-k, 0]$;

- Se $t=k+\left|W^{\prime \prime}\right| \leq j \leq|W|$, então

$$
F\left({ }_{0} W_{j}\right)=F\left(\left(W^{v^{\prime \prime}}\right)^{-1}\right)+F\left(W^{\prime}\right)+F\left({ }_{t} W_{j}\right)=-k+F\left({ }_{t} W_{j}\right)
$$


mas veja que ${ }_{t} W_{j}$ é um subpasseio de $\left(W^{\prime}\right)^{-1}$, e isto implica que $F\left({ }_{t} W_{j}\right) \in[0, k], \operatorname{logo} F\left({ }_{0} W_{j}\right) \in[-k, 0]$. Como queríamos.

Agora provaremos que $R_{k}^{-}(u) \subseteq D_{k}^{+}\left(R_{k}^{+}(v)\right)$. Seja $u^{\prime} \in R_{k}^{-}$. Considere um passeio $W^{\prime} \in R_{k}^{-}\left[u, u^{\prime}\right]$. Como o dígrafo $D$ tem grau de entrada pelo menos 1 , existe um vértice $v^{\prime \prime}$ e um passeio direcionado $W^{v^{\prime \prime}}$ de comprimento $k$ de $v^{\prime \prime}$ para $u^{\prime}$. Então o passeio $W=W^{v^{\prime}} \cdot W^{\prime} \cdot\left(W^{v^{\prime \prime}}\right)^{-1}$ está contido em $R_{k}^{+}\left[v^{\prime}, v^{\prime \prime}\right]$. Isto pode ser provado de forma similar ao que foi feito na demonstração da Afirmação 1. Portanto $v^{\prime \prime} \in R_{k}^{+}(v)$, e como $W^{v^{\prime \prime}}$ é um passeio direcionado de comprimento $k$ de $v^{\prime \prime}$ até $u, \operatorname{logo} u^{\prime} \in D_{k}^{+}\left(R_{k}^{+}(v)\right)$. A versão dual prova-se de forma análoga.

Proposição 3.14. Seja $D$ um dígrafo conexo, transitivo, localmente finito e seja $k \geq 1$ um inteiro. Suponha que, para algum (e consequentemente qualquer) $v \in V D$, pelo menos uma das classes $R_{k}^{+}(v)$ e $R_{k}^{-}(v)$ é finita. Então $R_{k}^{+}(v)$ e $R_{k}^{-}(v)$ são ambas finitas. Além disso,

$$
\frac{\left|R_{k}^{+}(v)\right|}{\left|R_{k}^{-}(v)\right|}=\left(\frac{d^{-}}{d^{+}}\right)^{k}
$$

Demonstração. Seja um vértice $v \in V D$. Do dígrafo $D$ ser localmente finito e conexo implica que $d^{+} \geq 1$ e $d^{-} \geq 1$, logo a Proposição 3.13 vale. Suponha sem perda de generalidade que $R_{k}^{+}(v)$ é finita. Considere um passeio direcionado de comprimento $k$ com vértice inicial $v$, e seja $u \in D_{k}^{+}(v)$ o vértice final deste passeio. Pela Proposição 3.13 temos que $D_{k}^{+}\left(R_{k}^{+}(v)\right)=R_{k}^{-}(u)$. Como $D$ é localmente finito e $R_{k}^{+}(v)$ é finita, então $D_{k}^{+}\left(R_{k}^{+}(v)\right)$ é finita. Logo $R_{k}^{-}(u)$ é finita (uma vez que, para cada $v^{\prime} \in R_{k}^{-}(u)$, existe um passeio direcionado de $W^{v^{\prime}}$ de comprimento $k$ de $v^{\prime}$ à $w \in D_{k}^{-}\left(R_{k}^{-}(u)\right)=R_{k}^{+}$, tal passeio existe pois $D$ é localmente finito). Do dígrafo $D$ ser transitivo e $R_{k}^{-}(u)$ um bloco, existe $g \in \operatorname{Aut}(D)$ tal que $\left(R_{k}^{-}(u)\right)^{g}=R_{k}^{-}(v), \operatorname{assim}\left|R_{k}^{-}(v)\right|=\left|R_{k}^{-}(u)\right|$. Portanto $R_{k}^{+}(v)$ é finita.

Finalmente mostraremos a igualdade 3.2. Pela Proposição 3.13 obtemos $D_{k}^{-}\left(R_{k}^{-}(u)\right)=R_{k}^{+}(v)$ e $D_{k}^{+}\left(R_{k}^{+}(v)\right)=R_{k}^{-}(u)$. Logo, todo passeio direcionado de comprimento $k$ começando em $R_{k}^{-}(u)$ termina em $R_{k}^{+}(v)$, e reciprocamente, todo passeio direcionado de comprimento $k$ começando em $R_{k}^{+}(v)$ termina em $R_{k}^{-}(u)$. Deste modo, o número de passeios iniciando em $R_{k}^{+}(v)$ é igual a $\left|R_{k}^{+}(v)\right|\left(d^{+}\right)^{k}$ e o número de passeios terminando em $R_{k}^{-}(u)$ é $\left|R_{k}^{-}(u)\right|\left(d^{-}\right)^{k}$. Portanto, $\left|R_{k}^{+}(v)\right|\left(d^{+}\right)^{k}=\left|R_{k}^{-}(u)\right|\left(d^{-}\right)^{k}=\left|R_{k}^{-}(v)\right|\left(d^{-}\right)^{k}$. 
Corolário 3.15. Seja D um dígrafo conexo, transitivo e localmente finito, seja $k \geq 1$ um inteiro. Suponha que para algum (e consequentemente qualquer) $v \in V D$, pelo menos uma das classes de equivalência $R_{k}^{+}(v)$ e $R_{k}^{-}(v)$ é finita. Então $\left|R_{k}^{+}(v)\right|=\left|R_{k}^{-}(v)\right|$ se, e somente se, $d^{+}=d^{-}$.

Demonstração. Segue diretamente da Proposição 3.14.

Lema 3.16. Seja $D$ um dígrafo conexo e transitivo, seja $k \geq 1$ um inteiro e um vértice $v \in V D$. Então para qualquer $u \in D_{k}^{+}(v)$ e $g \in A u t(D)$ temos que $g\left(R_{k}^{+}(v)\right)=R_{k}^{+}(v)$ se, e somente se, $g\left(R_{k}^{+}(u)\right)=R_{k}^{-}(u)$.

Demonstração. Sejam $v \in V D, u \in D_{k}^{+}(u)$ e $g \in \operatorname{Aut}(D)$. Suponha que $g\left(R_{k}^{+}(v)\right)=R_{k}^{+}(v)$. Sejam $W^{v}$ um passeio direcionado de comprimento $k$ de $v$ para $u$ e $W$ um passeio de $R_{k}^{+}[v, g(v)]$. Defina um novo passeio $W^{\prime}=\left(W^{v}\right)^{-1} . W \cdot g\left(W^{v}\right)$, então $W^{\prime} \in R_{k}^{-}[u, g(u)]$. Tal afirmação pode ser provada de forma análoga aos resultados anteriores. Então $g\left(R_{k}^{-}(u)\right)=R_{k}^{-}(u)$, pois $R_{k}^{-}(u)$ é um bloco de imprimitividade para $A u t(D)$. Portanto, $g \in A u t(D)$ deixa o bloco $R_{k}^{-}(u)$ invariante. A recíproca é provada de forma análoga.

O próximo resultado mostra uma condição necessária para um dígrafo conexo e transitivo ser um dígrafo de Cayley.

Proposição 3.17. Seja $D$ um dígrafo de Cayley conexo. Então para todo inteiro $k \geq 1$ e para todo $v \in V D$ temos que $\left|R_{k}^{+}(v)\right|=\left|R_{k}^{-}(v)\right|$.

Demonstração. Se $D$ é localmente finito então $d^{+}=d^{-}$, e do Corolário 3.15, obtemos $\left|R_{k}^{+}(v)\right|=$ $\left|R_{k}^{-}(v)\right|$. Suponha agora que $D$ é localmente infinito. Sejam $k \geq 1$ e $v \in V D$. Tome $u \in D_{k}^{+}(v)$. Seja $G$ um subgrupo do automorfismo de $D$ agindo regularmente sobre $D$, tal grupo existe pelo Lema 2.9. Pelo Lema 3.16 o bloco $R_{k}^{+}(v)$ é invariante para um automorfismo $g \in G$ se, e somente se, $g$ fixa o bloco $R_{k}^{-}(u)$. Então tais $g$ pertencem ao estabilizador $H=G_{\left\{R_{k}^{+}(v)\right\}}$. Do Lema 1.12 temos que $H$ age transitivamente sobre $R_{k}^{+}(v)$ e consequentemente $H$ age regularmente sobre $R_{k}^{+}(v)$. E do Teorema 1.3 obtemos $\left|R_{k}^{+}(v)\right|=|H|$. De forma análoga obtemos $\left|R_{k}^{-}(u)\right|=|H|$. Portanto, pela transitividade de $D,\left|R_{k}^{+}(v)\right|=\left|R_{k}^{-}(v)\right|$. 


\subsection{Propriedade Z e crescimento}

Em [5] Cameron, Praeger e Wormald demonstram alguns resultados a respeito das condições para um dígrafo ter propriedade $Z$, sendo um destes obtido através de relação de alcance nas arestas.

Nesta seção apresentamos três resultados: o primeiro dá uma condição necessária e suficiente para um dígrafo não ter propriedade $Z$, enquanto os demais nos dão uma condição suficiente para ter a propriedade $Z$. Lembrando que um dígrafo conexo, transitivo e infinito $D$ tem propriedade $Z$ se existe um epimorfismo de dígrafo $\phi: D \longrightarrow Z$, ou de forma equivalente, se todos os ciclos de $D$ são balanceados.

Lema 3.18. Seja $D$ um dígrafo infinito, conexo e transitivo. Então $D$ não tem propriedade $Z$ se, e somente se, existem inteiros $1 \leq s \leq t$, tais que para cada vértice $v \in V D$ existe, um ciclo $W^{v}$ em $v \operatorname{com} F\left(W^{v}\right)=$ s e $F\left({ }_{0} W_{j}^{s}\right) \in[0, t]$, para todo, $0 \leq j \leq\left|W^{v}\right|$.

Demonstração. Se existe tal passeio fechado $W^{v}$ então $W^{v}$ não é balanceado, portanto $D$ não tem propriedade $Z$.

Reciprocamente, suponha que $D$ não tem propriedade $Z$, logo existe em $D$ ciclos não balanceados. Seja $C=\left(v_{0}, \epsilon_{1}, v_{1}, \ldots, \epsilon_{n}\right)$ um ciclo, tal que $C$ é não balanceado e tem o menor comprimento. Como $C$ é não balanceado, temos que $F(C) \neq 0$, deste modo podemos considerar sem perda de generalidade que $s=F(C)>0$ (caso contrário tome o passeio $C^{-1}$ ). Seja $0 \leq i \leq n$ um inteiro tal que $F\left({ }_{0} C_{i}\right)=m$ é mínimo, com $m \in \mathbb{Z}$. Tome $u=v_{i}$.

Afirmação 1: O passeio fechado $W^{u}={ }_{i} C_{n} \cdot{ }_{0} C_{i}$ é tal que $F\left({ }_{0} W_{j}^{u}\right) \geq 0$, para todo $j$. De fato, suponha por contradição que existe um $0 \leq j_{1} \leq n$, tal que $F\left({ }_{0} W_{j_{1}}^{u}\right)<0$, digamos. Se $0 \leq j_{1} \leq n-i$ então

$$
F\left({ }_{0} C_{i+j_{1}}\right)=m+F\left({ }_{0} W_{j_{1}}^{u}\right)<m,
$$

contradição, pois $m$ é mínimo. Agora, se $n-i \leq j_{1} \leq n$, então

$$
F\left({ }_{0} W_{j_{1}}^{u}\right)=F\left({ }_{0} W_{n-i}^{u}\right)+F\left({ }_{n-i} W_{j_{1}}^{u}\right),
$$

porém $F\left({ }_{0} W_{n-i}^{u}\right)=F\left({ }_{i} C_{n}\right)$ e $F\left({ }_{n-i} W_{j_{1}}^{u}\right) \geq m$ (caso contrário, contradiz a minimalidade de $m$ ). 
Logo,

$$
0<s=m+F\left({ }_{i} C_{n}\right) \leq F\left({ }_{n-i} W_{j_{1}}^{u}\right)+F\left({ }_{0} W_{n-i}^{u}\right)=F\left({ }_{0} W_{j_{1}}^{u}\right)<0 .
$$

Contradição, portanto a afirmação é verdadeira.

Seja $t=\max \left\{F\left({ }_{0} W_{j}^{u}\right) \mid 0 \leq j \leq n\right\}$ o peso máximo. Assim pela Afirmação 1 obtemos $F\left({ }_{0} W_{j}^{u}\right) \in[0, t]$, para todo $0 \leq j \leq n$. Pela transitividade, um passeio $W^{w}$ com estas propriedades existe para cada $w \in V D$.

Usando o Lema 3.18, daremos agora duas condições suficientes dependendo da propriedade das relações $R_{k}^{+}$e $R_{k}^{-}$para o dígrafo $D$ ter propriedade $Z$.

Proposição 3.19. Seja D um dígrafo infinito, conexo, transitivo e localmente finito. Suponha que para cada inteiro $k \geq 1$ pelo menos uma (e consequentemente ambas) das relaçôes $R_{k}^{+}$e $R_{k}^{-}$ possui classe de equivalência finita. Então D tem propriedade Z.

Demonstração. Suponha por contradição que $D$ não tem propriedade Z. Sejam $1 \leq s \leq t$ inteiro dados no lema anterior, e tome $k=t$. Escolha $v \in V D$. Como por hipótese pelo menos uma das classes de equivalência é finita, segue da Proposição 3.14 que ambas as relações $R_{k}^{+}$e $R_{k}^{-}$tem classe de equivalência finita. Digamos $m=\left|R_{k}^{+}(v)\right|$. Seja $P=\left(v_{0}, 1, v_{1}, \ldots, 1, v_{m s}\right)$ um passeio direcionado de comprimento $m s$, começando em $v$. Vamos mostrar que os vértices $v_{0}, v_{s}, \ldots, v_{m s}$ são todos $R_{k}^{+}$-equivalentes. De fato, considere $W^{w}$ um ciclo não balanceado em $w \in V D$, dado pelo Lema 3.18. Defina o passeio $W^{\prime}=W^{v_{s}} \cdot\left({ }_{0} P_{s}\right)^{-1}$.

Afirmação 1: $W^{\prime}$ é um passeio de $R_{k}^{+}\left[v_{s}, v_{0}\right]$.

De fato, note que

$$
F\left(W^{\prime}\right)=F\left(W^{v_{s}}\right)+F\left(\left({ }_{0} P_{s}\right)^{-1}\right)=0
$$

e $F\left({ }_{0} W_{j}^{\prime}\right) \in[0, k]$ para todo $0 \leq j \leq\left|W^{\prime}\right|$. Basta observar que para $0 \leq j \leq\left|W^{v_{s}}\right|$ temos $F\left({ }_{0} W_{j}^{\prime}\right)=F\left({ }_{0} W_{j}^{v_{s}}\right) \in[0, k]$. Agora, se $\left|W^{v_{s}}\right| \leq j \leq\left|W^{\prime}\right|$, então

$$
F\left({ }_{0} W_{j}^{\prime}\right)=F\left({ }_{0} W^{v_{s}}\right)-s+i=s-s+i=i
$$

onde $i \in[0, s]$. Como $s \leq k$, segue que $F\left({ }_{0} W_{j}^{\prime}\right) \in[0, k]$.

Portanto, $W^{\prime} \in R_{k}^{+}\left[v_{s}, v_{0}\right]$. De forma análoga, prova-se que $W^{v_{s}}\left({ }_{s} P_{2 s}\right)^{-1}$ pertence a $R_{k}^{+}\left[v_{2 s}, v_{s}\right]$, 
etc. Logo,

$$
\left\{v_{0}, v_{s}, v_{2 s}, \ldots, v_{m s}\right\} \subseteq R_{k}^{+}(v)
$$

$\operatorname{Assim}\left|R_{k}^{+}(v)\right| \geq m+1$, uma contradição, pois $\left|R_{k}^{+}(v)\right|=m$. Portanto $D$ tem propriedade $Z$.

A próxima proposição mostra uma condição suficiente para um dígrafo ter propriedade $Z$ em termos dos expoentes.

Proposição 3.20. Seja $D$ um dígrafo infinito, conexo e transitivo. Se pelo menos um dos expoentes $\exp ^{+}(D)$ e $\exp ^{-}(D)$ é infinito, então $D$ tem propriedade $Z$.

Demonstração. Suponha que $D$ não tem propriedade $Z$. Sejam $1 \leq s \leq t$ inteiros dados pelo Lema 3.18 e denote $W^{w}$ o ciclo não balanceado correspondente a $w \in V D$. Tome $k=t$. Mostraremos que $R_{k+1}^{+}=R_{k+2}^{+}$, e isto implica, pela Proposição 3.7, que $R^{+}=R_{k+1}^{+}$.

Suponha que $u R_{k+2}^{+} v$, para algum $u, v \in V D$. Seja $W=\left(v_{0}, \epsilon_{1}, v_{1}, \ldots, \epsilon_{n}, v_{n}\right)$ o passeio de $R_{k+2}^{+}[u, v]$. Construiremos, agora um passeio $W^{\prime} \in R_{k+1}^{+}[u, v]$, através de ajustes em $W$, da seguinte forma. Seja $0<i<n$ o menor inteiro tal que $F\left({ }_{0} W_{i}\right)=k+2$ e seja $i \leq j \leq n$ o menor inteiro tal que $F\left({ }_{0} W_{j}\right)=s-1$. Note que se $i$ não existe, então o resultado segue. Agora, se $i$ existe então tal $j$ também existe pois, de $F\left({ }_{0} W_{i}\right)=k+2$ e $F(W)=0$, temos que para cada $0 \leq \alpha \leq k+2$ existe $i \leq j_{\alpha} \leq n$ tal que $F\left({ }_{0} W_{j_{\alpha}}\right)=\alpha$ (ou seja, o peso dos subpasseios deve decair até atingir o peso $\left.0=F\left({ }_{0} W_{n}\right)\right)$. Como $s-1 \leq k-1$, tome $\alpha=s-1$. Defina o passeio

$$
W^{\prime}={ }_{0} W_{i-1} \cdot\left(W^{v_{i-1}}\right)^{-1} \cdot{ }_{i-1} W_{j-1} \cdot W^{v_{j-1}} \cdot{ }_{j-1} W_{n} .
$$

De forma similar ao que foi feito nos resultados anteriores, obtemos que $W^{\prime} \in R_{k+1}^{+}[u, v]$. Além disso, temos também que $0<i^{\prime}<\left|W^{\prime}\right| \operatorname{com} F\left({ }_{0} W_{i^{\prime}}^{\prime}\right)=k+2$ é estritamente menor que o número de inteiros $0<i<|W|$ tal que $F\left({ }_{0} W_{i}\right)=k+2$. Continuando dessa maneira, isto é, a cada passo eliminando um "pico", obteremos um passeio de $u$ à $v$ pertencente ao conjunto $R_{k+1}^{+}[u, v]$. Como queríamos.

De forma análoga mostra que $R^{-}=R_{k+1}^{-}$. Portanto, as sequências $\left(R_{k}^{+}(v)\right)_{k \in \mathbb{Z}^{+}}$e $\left(R_{k}^{-}(v)\right)_{k \in \mathbb{Z}^{+}}$ são ambas finitas, uma contradição. Com isso provamos o resultado.

Finalmente, mostraremos que se pelo menos um dos expoentes $\exp ^{+}(D)$ ou $\exp ^{-}(D)$ é infinito, então $D$ tem crescimento exponencial. Pela Observação 2.12, é suficiente mostrar que 
temos uma cópia de $T^{*}$ em $D$.

Teorema 3.21. Seja $D$ um dígrafo conexo, transitivo e localmente finito. Se pelo menos um dos expoentes $\exp ^{+}(D)$ e $\exp ^{-}(D)$ é infinto. Então D tem crescimento exponencial.

Demonstração. Suponha sem perda de generalidade que $\exp ^{+}(D)$ é infinito. Pela Proposição 3.20, $D$ tem propriedade $Z$. Seja $\psi: D \rightarrow Z$ um homomorfismo sobrejetor e considere as fibras $F_{j}=\psi^{-1}(j), j \in \mathbb{Z}$. Primeiramente, veja que para todo $k \geq 1$, se $u \in F_{j}$ então $R_{k}^{+}(u) \subset F_{j}$. De fato, inicialmente observe que podemos supor que $u \in F_{0}$, pois $D$ é transitivo. Agora, seja $v \in R_{k}^{+}(u)$. Pelo Lema 3.2, existe um passeio em $R_{k}^{+}[u, v]$ que é formado por concatenações de passeios da forma

$$
W^{\prime}=\left(u_{0}, 1, u_{1}, 1, \ldots, 1, u_{k},-1, u_{k+1},-1, \ldots,-1, u_{2 k}\right) .
$$

Logo, temos

$$
u_{0}, u_{2 k} \in F_{0} ; u_{1}, u_{2 k-1} \in F_{1} ; \ldots ; u_{k-1}, u_{k+1} \in F_{k-1} ; u_{k} \in F_{k} .
$$

Assim $v \in F_{0}$, e portanto $R_{k}^{+}(u) \subseteq F_{0}$.

Afirmação. $\left|R_{1}^{+}(u)\right| \geq 2$.

Suponha que $\left|R_{1}^{+}(u)\right|=1$. Como $\left(R_{k}^{+}\right)_{k \in \mathbb{Z}^{+}}$é estritamente ascendente, existe $w \in R_{2}^{+}(u) \backslash R_{1}^{+}(u)$ tal que $\left(u, 1, u_{1}, 1, u_{2},-1, u_{3},-1, w\right)$ é um caminho em $D$. Como $w \notin R_{1}^{+}(u)$, segue que $u_{1} \neq u_{3}$. Logo $u_{1} R_{1}^{+} u_{3}$. Pela transitividade existe $v \neq u$ tal que $v R_{1}^{+} u$. Uma contradição. Portanto $\left|R_{1}^{+}(u)\right| \geq 2$.

Para cada $u \in F_{0}$ e cada $k \geq 1$, defina $C_{u}^{k}$ o subdígrafo de $D$ induzido por todos os passeios $W \in R_{k}^{+}\left[u^{\prime}, u^{\prime \prime}\right]$, onde $u^{\prime}, u^{\prime \prime} \in R_{k}^{+}(u)$. Note que, os vértices de $C_{u}^{k}$ estão contidos em $\bigcup_{i=0}^{k} F_{i}$, pois para qualquer $W \in R_{k}^{+}\left[u^{\prime}, u^{\prime \prime}\right]$, temos $W \subset \bigcup_{i=0}^{k} F_{i}$. De $R_{k}^{+} \subset R_{k+1}^{+}$segue que $C_{u}^{k}$ está propriamente contido em $C_{u}^{k+1}$. As árvore binárias serão construídas indutivamente da seguinte forma. Primeiro, note que: 
Afirmação. $\left|R_{1}^{+}(u)\right| \geq 2$.

Suponha que $\left|R_{1}^{+}(u)\right|=1$. Como $\left(R_{k}^{+}\right)_{k \in \mathbb{Z}^{+}}$é estritamente ascendente, existe $w \in R_{2}^{+}(u) \backslash R_{1}^{+}(u)$ tal que $\left(u, 1, u_{1}, 1, u_{2},-1, u_{3},-1, w\right)$ é um caminho em $D$. Como $w \notin R_{1}^{+}(u)$, segue que $u_{1} \neq u_{3}$. Logo $u_{1} R_{1}^{+} u_{3}$. Pela transitividade existe $v \neq u$ tal que $v R_{1}^{+} u$. Uma contradição. Portanto $\left|R_{1}^{+}(u)\right| \geq 2$.

Consequentemente cada vértice de $F_{1}$ tem grau de entrada pelo menos 2. Assim, pela transitividade de $D$, cada vértice de $F_{1}$ é a raiz de uma árvore binária de altura 1 que está contida em algum $C_{u}^{1}$. Agora, cada $C_{u}^{2}$ contém pelo menos dois subdígrafos distintos em $\left\{C_{u^{\prime}}^{1} \mid u^{\prime} \in F_{0}\right\}$, digamos $C_{u_{1}}^{1}$ e $C_{u_{2}}^{1}$. Assim existem vértices $w_{1} \in C_{u_{1}}^{1} \cap F_{1}, w_{2} \in C_{u_{2}}^{1} \cap F_{1}$ e $v \in C_{u}^{2} \cap F_{2}$ tal que $\left(w_{1}, v\right),\left(w_{2}, v\right) \in E D$. Segue que $v$ é uma raiz de uma árvore binária de altura 2 , que está contida em algum $C_{u}^{2}$, pois cada $w_{1}$ e $w_{2}$ é raiz de uma árvore de altura 1. Pela transitividade de $D$ obtemos que todo vértice de $F_{2}$ é uma raiz de uma árvore binária de altura 2, que está contida em algum $C_{u}^{2}$.

Temos então o passo de indução, cada $C_{u}^{k+1}, k \geq 1$, contém pelo menos dois diferentes subdígrafos de $\left\{C_{u^{\prime}}^{k} \mid u^{\prime} \in F_{0}\right\}$, digamos $C_{u_{1}}^{k}$ e $C_{u_{2}}^{k}$. Logo, existem vértices $w_{1} \in C_{u_{1}}^{k} \cap F_{k}$, $w_{2} \in C_{u_{2}}^{k} \cap F_{k}$ e $v \in C_{u}^{k+1} \cap F_{k+1}$ tal que $\left(w_{1}, v\right),\left(w_{2}, v\right) \in E D$ (tais vértices existem uma vez que por suposição, $C_{u_{1}}^{k}$ e $C_{u_{2}}^{k}$, estão ambos contidos em $C_{u}^{k+1}$ ). Por hipótese de indução $w_{1}$ e $w_{2}$ são raízes de uma árvore binária de altura $k$ que são disjuntas pois $C_{u_{1}}^{k}$ e $C_{u_{2}}^{k}$ são diferentes. Segue que $v$ é a raiz de uma árvore binária de altura $k+1$ que está contida em $C_{u}^{k+1}$. De $D$ ser transitivo temos que todo vértice de $F_{k+1}$ é uma raiz de uma árvore binária de altura $k+1$, que está contida em algum $C_{u}^{k+1}$. Por indução temos árvores binárias de altura arbitrariamente grande em $D$. 


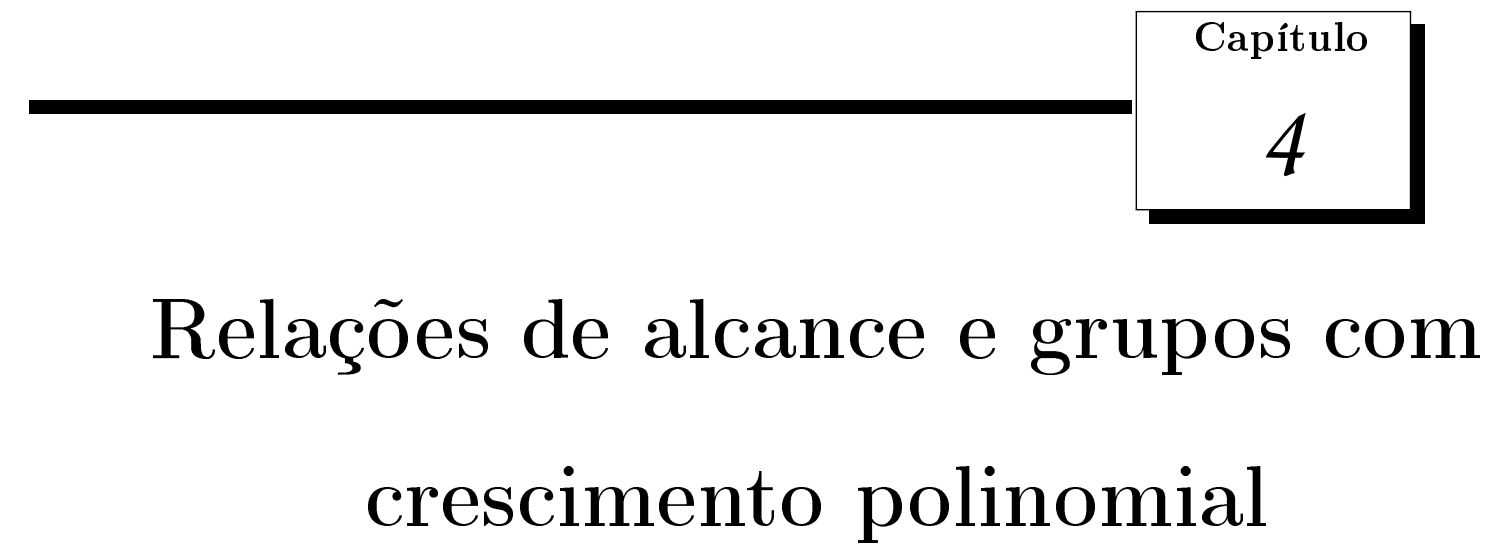

Mostramos no capítulo 3, que as relações de alcance estão intimamente ligadas com propriedades de dígrafos tal como propriedade $Z$ e condições de crescimento.

Neste capítulo apresentamos através de resultados provados em [13] as conexões entre estas relações com certas propriedades de grupo de automorfismo de dígrafos transitivos. Inicialmente apresentaremos alguns resultados que servirão de base para a próxima seção. Em seguida, estudaremos dígrafos de Cayley de grupos finitamente gerado com crescimento polinomial, e finalmente apresentamos o teorema principal deste trabalho

Teorema 4.15. Seja $G$ um grupo agindo transitivamente sobre um digrafo conexo $D$ e seja $N \unlhd G$ um subgrupo nilpotente de classe $r$ agindo com $m$ órbitas sobre $D$, onde $1 \leq m<\infty$. Então $\exp ^{+}(D)=\exp ^{-}(D) \leq m(r+1)-1$.

Consequentemente, temos

Corolário 4.16. Seja $G$ um grupo finitamente gerado, seja $N$ um subgrupo normal nilpotente de indice finito $m$ em $G$ e seja $D$ o dígrafo de Cayley de $G$ com respeito a algum conjunto finito de geradores $S$. Então $\exp ^{+}(D)=\exp ^{-}(D) \leq m(r+1)-1$ vale, onde $r$ é a classe de nilpotência 
de $N$.

Finalizaremos este capítulo apresentando uma conjectura que caso seja verdadeira fornece uma resposta positiva para o seguinte problema de Grigorchuk.

Problema 4.2. Todo grupo simples, infinito e finitamente gerado tem crescimento exponencial?

\subsection{Resultados auxiliares}

O objetivo principal desta seção é apresentar resultados e definições que serão usados na Seção 4.2. Consideraremos agora quocientes de um dígrafo com respeito a uma classe de equivalência em seus vértices.

Seja $D$ um dígrafo e seja $\tau$ uma partição do conjunto de vértices $V D$, dada por alguma relação de equivalência definida em $V D$. A classe de equivalência de um vértice $v \in V D$ será denotada por $v^{\tau}$. O dígrafo quociente com respeito à $\tau$, denotado por $D_{\tau}$, é o dígrafo que tem como vértices o conjunto $\tau$ e o conjunto de arestas com a seguinte condição:

$$
\left(u^{\tau}, v^{\tau}\right) \in E D_{\tau} \Leftrightarrow \exists u^{\prime} \in u^{\tau} \text { e } v^{\prime} \in v^{\tau} \text { tal que }\left(u^{\prime}, v^{\prime}\right) \in E D
$$

Enfatizamos que consideraremos estes dígrafos como dígrafos simples no sentido que se existem mais de uma aresta na mesma direção entre dois conjuntos de $\tau$, então o dígrafo quociente contém exatamente uma aresta entre os respectivos vértices. Observe que estes dígrafos quocientes podem conter laço se existir uma aresta $(u, v) \in E D$ para algum $u \in v^{\tau}$.

Seja $W=\left(v_{0}, \epsilon_{1}, v_{1}, \ldots, \epsilon_{n}, v_{n}\right)$ um passeio em $D$. Definimos como passeio quociente $W_{\tau}$ de $W$ o passeio

$$
W_{\tau}=\left(v_{0}^{\tau}, \epsilon_{1}, v_{1}^{\tau}, \ldots, \epsilon_{n}, v_{n}^{\tau}\right)
$$

Note que para todo $j, 0 \leq j \leq\left|W^{\tau}\right|$, temos que $F\left({ }_{0}\left(W_{\tau}\right)_{j}\right)=F\left({ }_{0} W_{j}\right)$.

Os dois próximos resultado mostram como $R_{k}^{+}$(respectivamente $R_{k}^{-}$) sobre $D$ estão relacionadas com $R_{k}^{+}$(respectivamente $R_{k}^{-}$) sobre $D_{\tau}$, onde $\tau$ é uma partição dada por $R_{1}^{+}$(respectivamente $R_{1}^{-}$). Resultados adicionais sobre dígrafos quocientes com respeito à relações $R_{k}^{+}$e $R_{k}^{-}$ podem serem encontrados em [18]. 
Proposição 4.1. Seja D um dígrafo, seja $\tau$ o conjunto das classes de equivalências $R_{1}^{+}$, e seja $u \in V D$. Então, para quaisquer $v \in V D$ e $k \geq 2$ temos que $u R_{k}^{+} v$ se, e somente se, $u^{\tau} R_{k-1}^{+} v^{\tau}$. Uma afirmação análoga vale para $R_{k}^{-}$, quando consideramos o dígrafo quociente com respeito à $R_{1}^{-}$.

Demonstração. Seja $W=\left(v_{0}, \epsilon_{1}, v_{1}, \ldots, \epsilon_{n}, v_{n}\right)$ um passeio do conjunto $R_{k}^{+}[u, v]$. Logo por definição, existe em $D_{\tau}$ o passeio

$$
W_{\tau}=\left(v_{0}^{\tau}, \epsilon_{1}, v_{1}^{\tau}, \ldots, \epsilon_{n}, v_{n}^{\tau}\right) \in R_{k}^{+}\left[u^{\tau}, v^{\tau}\right]
$$

Mostraremos que $u^{\tau} R_{k-1}^{+} v^{\tau}$, através de modificações no passeio $W_{\tau}$ obteremos um novo passeio no dígrafo quociente, onde tal passeio pertencerá à $R_{k-1}^{+}\left[u^{\tau}, v^{\tau}\right]$. Seja $0<j<n$ o menor inteiro tal que $F\left({ }_{0} W_{j}\right)=k$. Se $j$ não existe, então $F\left({ }_{0} W_{j}\right)<k$, portanto $u^{\tau} R_{k-1}^{+} v^{\tau}$. Assuma que $j$ existe. Considere o subpasseio ${ }_{j-1} W_{j+1}$. Sabemos que $F\left({ }_{0} W_{j}\right)=F\left({ }_{0} W_{j-1}\right)+\epsilon_{j}$ e por suposição $F\left({ }_{0} W_{j}\right) \leq k$, segue que $\epsilon_{j}=1$. Analogamente obtemos que $\epsilon_{j+1}=-1$. De onde concluímos que ${ }_{j-1} W_{j+1}$ é um passeio em $R_{1}^{+}\left[v_{j-1}, v_{j+1}\right]$, isto é, $v_{j-1} R_{1}^{+} v_{j+1}$, daí $v_{j-1}^{\tau}=v_{j+1}^{\tau}$. Neste caso, substituiremos o passeio $W_{\tau}$ pelo passeio

$$
W^{\prime}={ }_{0}\left(W_{\tau}\right)_{j-1} \cdot{ }_{j+1}\left(W_{\tau}\right)_{n}
$$

Se ainda existir algum $0<j^{\prime}<\left|W^{\prime}\right|$ tal que $F\left({ }_{0} W_{j^{\prime}}\right)=k$, repetimos o procedimento acima. Continuando dessa maneira e observando que $j<j^{\prime}<\left|W^{\prime}\right|$, obtemos um passeio pertencente à $R_{k-1}^{+}\left[u^{\tau}, v^{\tau}\right]$. Como queríamos.

Reciprocamente, seja

$$
\bar{W}=\left(u_{0}^{\tau}, \epsilon_{1}, u_{1}^{\tau}, \ldots, \epsilon_{n}, u_{n}^{\tau}\right)
$$

um passeio no dígrafo quociente tal que $u \in u_{0}^{\tau}, v \in u_{n}^{\tau}$ e $\bar{W} \in R_{k-1}^{+}\left[u_{0}^{\tau}, u_{n}^{\tau}\right]$. Por definição, para cada $1 \leq j \leq n$ existem vértices $u_{j-1}^{\prime} \in u_{j-1}^{\tau}$ e $u_{j}^{\prime \prime} \in u_{j}^{\tau}$ tal que $\left(u_{j-1}^{\prime}, \epsilon_{j}, u_{j}^{\prime \prime}\right)$ é um passeio em $D$ e $\left(u_{j-1}^{\tau}, \epsilon_{j}, u_{j}^{\tau}\right)$ é um passeio no dígrafo quociente. Faça $u_{0}^{\prime \prime}=u$ e $u_{n}^{\prime}=v$. Observe que para $0 \leq j \leq n$, temos $u_{j}^{\prime}, u_{j}^{\prime \prime} \in u_{j}^{\tau}$, o que significa que $u_{j}^{\prime} R_{1}^{+} u_{j}^{\prime \prime}$. Para cada $0 \leq j \leq n$, seja 
$W^{j} \in R_{1}^{+}\left[u_{j}^{\prime \prime}, u_{j}^{\prime}\right]$. Portanto o passeio

$$
W=W^{0} \cdot\left(u_{0}^{\prime}, \epsilon_{1}, u_{1}^{\prime \prime}\right) \cdot W^{1} \cdot\left(u_{1}^{\prime}, \epsilon_{2}, u_{2}^{\prime \prime}\right) \ldots W^{n-1} \cdot\left(u_{n-1}^{\prime}, \epsilon_{n}, u_{n}^{\prime \prime}\right) \cdot W^{n} \in R_{k}^{+}[u, v]
$$

Assim, concluímos que $u R_{k}^{+} v$. Analogamente é provado para $R_{k}^{-}$.

Seja $\tau$ uma partição do conjunto $V D$ e considere a seguinte condição:

(*) para cada $u, v \in V D \operatorname{com}\left(u^{\tau}, v^{\tau}\right) \in E D_{\tau}$ existe $u^{\prime} \in u^{\tau}$ e $v^{\prime} \in v^{\tau}$ tal que $\left(u, v^{\prime}\right)$ e $\left(u^{\prime}, v\right)$ são arestas de D.

Lema 4.2. Seja $D$ um dígrafo e seja $\tau$ uma partição do conjunto de vértice de D. Suponha que $D$ satisfaça a condição $(*)$. Então para cada $k \geq 1$ e cada $u, v \in V D$ temos que $u^{\tau} R_{k}^{+} v^{\tau}$ se, e somente se, existe algum $w \in v^{\tau}$ tal que $u R_{k}^{+} w$. Um resultado análogo vale para a relação $R_{k}^{-}$.

Demonstração. Sejam $u, v \in V D$ e seja $k \geq 1$ um inteiro. Suponha que para algum $w \in v^{\tau}$, temos que $u R_{k}^{+} w$. Logo existe um passeio $W \in R_{k}^{+}[u, w]$ e consequentemente, $W_{\tau} \in R_{k}^{+}\left[u^{\tau}, w^{\tau}\right]$. Como $v^{\tau}=w^{\tau}$, seque que $W_{\tau} \in R_{k}^{+}\left[u^{\tau}, v^{\tau}\right]$. Portanto, $u^{\tau} R_{k}^{+} v^{\tau}$.

Reciprocamente, suponha que $u^{\tau} R_{k}^{+} v^{\tau}$ e seja

$$
\bar{W}=\left(u^{\tau}, \epsilon_{1}, \bar{x}_{1}, \ldots, \epsilon_{n}, \bar{x}_{n}, \epsilon_{n+1}, v^{\tau}\right)
$$

um passeio em $R_{k}^{+}\left[u^{\tau}, v^{\tau}\right]$. Então por hipótese podemos encontrar sucessivamente representantes $x_{i} \in \bar{x}_{i}$ e $w \in v^{\tau}$ tal que

$$
W=\left(u, \epsilon_{1}, x_{1}, \epsilon_{2}, x_{2}, \ldots, \epsilon_{n}, x_{n}, \epsilon_{n+1}, w\right)
$$

é um passeio em $R_{k}^{+}[u, w]$.

Seja $G$ um grupo agindo transitivamente sobre $D$ e seja $H$ um subgrupo normal de $G$. Conforme visto no capítulo 2 (veja Teorema 1.6), as órbitas de $H$ formam um sistema de imprimitividade $\tau$ de $G$ sobre $V D$. O respectivo dígrafo quociente $D_{\tau}$ será denotado por $D_{H}$.

Lema 4.3. Seja $D$ um dígrafo e $H \leq A u t(D)$. Então a condição (*) é satisfeita. 
Demonstração. Seja $H \leq A u t(D)$. Daí, para $u, v \in V D \operatorname{com}\left({ }^{H} u,{ }^{H} v\right) \in E D_{H}$, por definição, existe $a \in{ }^{H} u, b \in{ }^{H} v$ tal que $(a, b) \in E D$. De $a \in{ }^{H} u$ e $b \in{ }^{H} v$ existe $x, y \in H$ tal que, $a={ }^{x} u$ e $b={ }^{y} v$. Logo,

$$
(a, b)=\left({ }^{x} u,{ }^{y} v\right) \in E D \Leftrightarrow{ }^{x^{-1}}\left({ }^{x} u,{ }^{y} v\right)=\left(u,{ }^{x^{-1} y} v\right) \in E D
$$

e

$$
(a, b)=\left({ }^{x} u,{ }^{y} v\right) \in E D \Leftrightarrow{ }^{y^{-1}}\left({ }^{x} u,{ }^{y} v\right)=\left({ }^{y^{-1} x} u, v\right) \in E D .
$$

Agora basta tomar $u^{\prime}=y^{-1} x u$ e $v^{\prime}={ }^{-1} y v$.

Corolário 4.4. Seja $D$ um dígrafo e $H \leq A u t(D)$. Então para cada $k \geq 1$ e cada $u, v \in V D$ temos que ${ }^{H} u R_{k}^{+}{ }^{H} v$ se, e somente se, existe algum $w \in{ }^{H} v$ tal que $u R_{k}^{+} w$. Um resultado análogo vale para a relaçẫo $R_{k}^{-}$.

Demonstração. Segue imediatamente dos Lemas 4.3 e 4.2 .

O seguinte resultado mostra que o expoente de um dígrafo é limitado pelo expoente do seu dígrafo quociente. Lembrando que o expoente $\exp ^{+}(D)$, de um dígrafo $D$ (veja página 29), é o menor inteiro $k \geq 1$ tal que $R^{+}=R_{k}^{+}$e se $R^{+} \neq R_{k}^{+}$para todo $k$ então $\exp ^{+}(D)=\infty$. Definição similar para o expoente $\exp ^{-}(D)$. .

Lema 4.5. Seja $G$ um grupo agindo transitivamente sobre um dígrafo $D$ e seja $H \unlhd G$, tal que todos subgrupos de $H$ são normais em $G$. Então $\exp ^{+}(D) \leq \exp ^{+}\left(D_{H}\right)+1 e$ $\exp ^{-}(D) \leq \exp ^{-}\left(D_{H}\right)+1$.

Demonstração. Provaremos o resultado para $\exp ^{+}(D)$. Se $\exp ^{+}\left(D_{H}\right)=\infty$, não há o que provar. Assim, assuma que $\exp ^{+}\left(D_{H}\right)=k$ para algum inteiro $k \geq 1$, daí mostraremos que $\exp ^{+}(D) \leq k+1$.

Sejam $u \in V D$ e $v \in R^{+}(u)$. Considere a classe de equivalência $B=R_{k+1}^{+}(u)$ e a $H$-órbita de $u,{ }^{H} u$. Pelo que já foi mostrado, temos que estes conjuntos são blocos de imprimitividade pela ação de $G$ sobre $V D$. Tome $N=H_{\{B\}}$, o estabilizador de $B$ em $H$.

Afirmação 1: Dois vértices dentro da mesma $H$-órbita estão $R_{k+1}^{+}$-relacionados se eles pertencem a mesma $N$-órbita. 
De fato, mostraremos inicialmente que ${ }^{N} u={ }^{H} u \cap B$. Seja $x \in{ }^{N} u$, então $x={ }^{n} u$, para algum $n \in N$. Como $N \leq H$ segue que ${ }^{n} u \in{ }^{H} u$ e de $u \in B$ e $n \in N$ obtemos que ${ }^{n} u \in B$. Portanto, ${ }^{N} u \subseteq{ }^{H} u \cap B$. Agora, suponha por contradição que ${ }^{H} u \cap B \nsubseteq{ }^{N} u$, ou seja, existe $y \in{ }^{H} u \cap B$ tal que $y \notin{ }^{N} u$. Como $y \in{ }^{H} u$, então $y={ }^{h} u$, para algum $h \in H \backslash N$. Por outro lado, $u \in B$ implica que ${ }^{h} u \in{ }^{h} B$, assim $y \in{ }^{h} B \cap B$ e como $B$ é um bloco segue que $B={ }^{h} B$, logo $h \in N$, contradição. Portanto ${ }^{H} u \cap B \subseteq{ }^{N} u$. Logo ${ }^{N} u={ }^{H} u \cap B$. E do exemplo 1.9 temos que a $N$-órbita é um bloco de imprimitividade para $G$. Como por hipótese, $N$ é um subgrupo normal em $G$, do Teorema 1.6, as $N$-órbitas formam um sistema de blocos, e este coincide com o sistema de blocos gerados pelo bloco ${ }^{N} u$. Isto prova a afirmação.

Primeiro mostraremos que $\exp ^{+}\left(D_{N}\right) \leq k$. Suponha que não, então existe ${ }^{N} w \in V D_{N}$ tal que ${ }^{N} w \in R_{k+1}^{+}\left({ }^{N} u\right) \backslash R_{k}^{+}\left({ }^{N} u\right)$. Pelo Corolário 4.4 existe algum $w^{\prime} \in{ }^{N} w$ tal que $u R_{k+1}^{+} w^{\prime}$. Isto implica também pelo Corolário 4.4 que ${ }^{H} u R_{k+1}^{+}{ }^{H} w$. Agora, como $\exp ^{+}\left(D_{H}\right)=k$, temos $R_{k+1}^{+}=R_{k}^{+}$em $D_{H}$ e segue que ${ }^{H} u \in R_{k}^{+}\left({ }^{H} w\right)$. Novamente pelo Corolário 4.4, existe $z \in{ }^{H} w$ tal que $u R_{k}^{+} z$. Da transitividade de $R_{k+1}^{+}$, segue que $z R_{k+1}^{+} w^{\prime}$, pois $u R_{k+1}^{+} w^{\prime}$ e $u R_{k+1}^{+} z$. Assim, como $w, z \in{ }^{H} w$, segue da Afirmação 1 que ${ }^{N} z={ }^{N} w^{\prime}$. Como $z R_{k}^{+} u$ temos ${ }^{N} z \in R_{k}^{+}\left({ }^{N} u\right)$. Logo ${ }^{N} w \in R_{k}^{+}\left({ }^{N} u\right)$, pois $w^{\prime} \in{ }^{N} w$, uma contradição. Deste modo, concluímos que $\exp ^{+}\left(D_{N}\right) \leq k$.

Como $v R^{+} u$ segue que ${ }^{N} v \in R_{k}^{+}\left({ }^{N} u\right)$ em $D_{N}$, e pelo Corolário 4.4, existe algum $x \in{ }^{N} v$ tal que $u R_{k+1}^{+} x$. Como $x, v \in{ }^{N} v$ e da afirmação, temos que $x R_{k+1}^{+} v$. Segue então da transitividade que $u R_{k+1}^{+} v$. Como $u$ e $v$ são arbitrários, concluímos que $\exp ^{+}(D) \leq k+1$.

De forma análoga, prova-se que $\exp ^{-}(D) \leq \exp ^{-}\left(D_{H}\right)+1$.

Trofimov e Seifter mostraram em [18] que o dígrafo quociente de um dígrafo com respeito à $R^{+}$e $R^{-}$podem ser descrito facilmente, como veremos a seguir. A demonstração do próximo resultado pode ser encontrada em [[18], Corolário 2.7].

Corolário 4.6. Seja D um dígrafo transitivo. Então as seguintes afirmações valem:

(a) O dígrafo quociente $D / R^{+}$é um dos seguintes dígrafos: um ciclo direcionado finito ou o dígrafo $Z$ ou uma árvore infinita direcionada regular com grau de entrada 1 e grau de saída maior do que 1.

(b) o dígrafo quociente $D / R^{-}$é um dos seguintes dígrafos: um ciclo direcionado finito ou o 
dígrafo $Z$ ou uma árvore regular direcionada com grau de saída 1 e grau de entrada maior do que 1.

Com isso, podemos provar o seguinte resultado:

Lema 4.7. Seja $G$ um grupo agindo transitivamente sobre um digrafo $D$ com expoentes exp ${ }^{+}(D)$ e $\exp ^{-}(D)$ ambos finitos. Além disso, seja $\tau$ o sistema de imprimitividade de G sobre VD induzido pelas classes de equivalência com respeito à $R^{+}$ou $R^{-}$. Então todo $g \in G$ que deixa invariante pelo menos um bloco de $\tau$ deixa invariante todos blocos de $\tau$.

Demonstração. Por hipótese, $\exp ^{+}(D)$ e $\exp ^{-}(D)$ são ambos finitos, logo pelo Corolário 3.10 segue que $R^{+}=R^{-}$, daí o Corolário 4.6 implica que o dígrafo quociente $D_{\tau}$ é um ciclo finito ou um caminho direcionado infinito bilateral. Consequentemente, o único automorfismo de $D_{\tau}$ que fixa um vértice é a identidade. Por outro lado, todo automorfismo $g \in G$ que deixa invariante um bloco de $\tau$, induz um automorfismo de $D_{\tau}$ fixando um vértice de $D_{\tau}$. Como os vértices de $D_{\tau}$ são os elementos de $\tau$ então $g$ induz a identidade em $D_{\tau}$. Isso implica que $g$ fixa todos os blocos de $\tau$.

Observe que, pelo lema anterior, as classes de equivalências da relação $R^{+}=R^{-}$são órbitas para um subgrupo normal de $G$. De fato, seja $H:=G_{\{\Delta\}}$, onde $\Delta$ é um bloco de $\tau$. Note que $\Delta$ é uma órbita para $G_{\{\Delta\}}$. Logo, basta mostrar que $H$ é normal em $G$, ou seja, $g^{-1} H g=H$, para todo $g \in G$. Seja $h \in H$, pelo Lema 4.7 temos $h \in G_{\left\{{ }^{g} \Delta\right\}}$ para todo $g \in G$. Dessa forma, é suficiente mostrar que $G_{\left\{{ }^{g} \Delta\right\}}=g^{-1} H g$, para todo $g \in G$. Seja $\left.x \in G_{\{g} \Delta\right\}$, mostraremos que $x \in g^{-1} H g$, isto é, $x=g^{-1} y g$ para algum $y \in H$. Seja $u \in \Delta$. Então ${ }^{x}\left({ }^{g} u\right) \in{ }^{g} \Delta$, assim ${ }^{x g} u={ }^{g} v$ para algum $v \in \Delta$. E isto implica que $v=g^{-1} x g$. Portanto $x \in g^{-1} H g$. De forma análoga, prova que $\left.g^{-1} H g \subseteq G_{\{g} \Delta\right\}$. Como queríamos.

\subsection{As relações $R^{+}$e $R^{-}$em dígrafos transitivos}

Começaremos com uma proposição sobre dígrafo de Cayley de um grupo abeliano.

Proposição 4.8. Seja $G$ um grupo abeliano agindo transitivamente sobre um dígrafo D. Então $\exp ^{+}(D)=\exp ^{-}(D)=1$. 
Demonstração. Pelo Corolário 2.10 D é um dígrafo de Cayley de $G$. Tomando $k=1$ no Corolário 3.3 , temos que para todo $g \in G$

$$
\begin{aligned}
R_{1}^{+}(g) & =g\left\langle S . S^{-1}\right\rangle \\
\mathrm{e} & \\
R_{1}^{-}(g) & =g\left\langle S^{-1} \cdot S\right\rangle
\end{aligned}
$$

e lembrando que $G$ é abeliano, logo $S . S^{-1}=S^{-1} S$. Assim, temos que $R_{1}^{+}=R_{1}^{-}$, e do Corolário 3.9 segue que $R^{+}=R_{1}^{+}=R_{1}^{-}=R^{-}$. Portanto $\exp ^{+}(D)=\exp ^{-}(D)=1$.

Note que um grupo abeliano é nilpotente de classe 1, então será que a proposição acima pode ser generalizada para grupos nilpotentes de classe $r$ ? Sim, como podemos ver no próximo teorema.

Teorema 4.9. Seja $G$ um grupo nilpotente de classe $r$ agindo transitivamente sobre um dígrafo D. Então $\exp ^{+}(D)=\exp ^{-}(D) \leq r$.

Demonstração. Primeiramente, mostraremos que $\exp ^{+}(D) \leq r$. A prova será feita por indução sobre $r$. Se $r=1$ então $G$ é abeliano, daí da Proposição 4.8, $\exp ^{+}(D)=1$.

Suponha agora que $r \geq 2$. Assim $\gamma_{r+1}(G)=\left[\gamma_{r}(G), G\right]=1$, isto implica que se tomarmos $H=\gamma_{r}(G)$ então $H$ está contido no centro de $G$, consequentemente cada subgrupo de $H$ é normal em $G$. Logo, do Lema 4.5, temos $\exp ^{+}(D) \leq \exp ^{+}\left(D_{H}\right)+1$. Agora, observe que o grupo quociente $\frac{G}{H}$ é nilpotente de classe $r-1$ e age transitivamente sobre o dígrafo quociente $D_{H}$ (pois $G$ age transitivamente sobre $D$ ). Então, por hipótese de indução, temos

$$
\exp ^{+}\left(D_{H}\right) \leq r-1
$$

consequentemente, pelo Lema 4.5,

$$
\exp ^{+}(D) \leq \exp ^{+}\left(D_{H}\right)+1 \leq r
$$

De forma análoga obtemos que $\exp ^{-}(D) \leq r$. Portanto, do Corolário 3.10, temos que $\exp ^{+}(D)=$ $\exp ^{-}(D) \leq r$. 
O próximo exemplo mostra que a cota do teorema acima é a melhor possível, isto é, para todo inteiro positivo $r$ existe um grupo nilpotente $G$ de classe $r$ e um dígrafo $D$, com $G$ agindo transitivamente sobre $D$ tal que $\exp ^{+}(D)=r=\exp ^{-}(D)$ vale.

Exemplo 4.10. Se $r=1$ então pela Proposição 4.8, $\exp ^{+}(D)=\exp ^{-}(D)=1$. Agora, para $r=2$. Considere, o grupo diedral

$$
D_{4}=\left\langle f, a_{1}, a_{2} \mid f^{2}=a_{1}^{2}=a_{2}^{2}=1, f a_{1} f^{-1}=a_{1} a_{2}, f a_{2}=a_{2} f, a_{1} a_{2}=a_{2} a_{1}\right\rangle .
$$

Então para o dígrafo de $D=C a y\left(D_{4}, S\right)$, onde $S=\left\{f, f a_{1}\right\}$, claramente temos que $\exp ^{+}(D)=\exp ^{-}(D)=2$. Na verdade, este exemplo é o menor elemento da seguinte família infinita.

Seja $n \geq 1$ um inteiro e seja $G_{n}$ o produto semidireto de um grupo elementar abeliano $\mathbb{Z}_{2}^{n}$ por um grupo cíclico $\mathbb{Z}_{2^{n-1}}$ dado por $G_{n}=\left\langle f, a_{1}, a_{2}, a_{3}, \ldots, a_{n}\right\rangle$ onde $f$ é de ordem $2^{n-1}$, os $a_{i}$ são involuçôes comutando com cada outro e $f a_{i} f^{-1}=a_{i} a_{i+1}$ para todo $i, 1 \leq i<$ $n$, enquanto $f$ e $a_{n}$ comutam. Note que, se considerarmos $S=\left\{f, f a_{1} a_{2} \ldots a_{n-1}\right\}$ obtemos que $\left\langle S^{i} S^{-i}\right\rangle=\left\langle a_{1}, a_{2}, \ldots, a_{i}\right\rangle$ vale, para todo $1 \leq i \leq n$, assim segue do Corolário 3.3 que $\exp ^{+}\left(\operatorname{Cay}\left(G_{n}, S\right)\right)=n$. Além disso, observe que $\gamma_{i}\left(G_{n}\right)=\left\langle a_{i}, a_{i+1}, \ldots, a_{n}\right\rangle$ vale para cada $i$, $2 \leq i \leq n-1$, consequentemente $\gamma_{n+1}\left(G_{n}\right)=1$, portanto $G_{n}$ é nilpotente de classe $n$.

O próximo exemplo mostra que o Teorema 4.9 não pode ser generalizado para grupos solúveis. Exemplo 4.11. O grupo Lamplighter L é o produto entrelaçado $\mathbb{Z}_{2} \imath \mathbb{Z}$ dado por

$$
L=\left\langle a, t \mid a^{2},\left[t^{m} a t^{-m}, t^{n} a t^{-n}\right], m, n \in \mathbb{Z}\right\rangle
$$

Considere o dígrafo de Cayley de $L$ com respeito ao conjunto de geradores $S=\{t$, at $\}$. De acordo os resultados em [20] o dígrafo de Cayley é o produto horocíclico de duas árvores infinitas com grau de entrada 1 e grau de saída 2 e tal produto é uma árvore regular com grau de entrada 2 e grau de saída 2 (veja [3]). Assim, do Exemplo 3.11, segue que $R^{+} \neq R_{k}^{+}$, para todo inteiro $k \geq 1$. Com isto, mostramos que o Teorema 4.9 não pode ser generalizado para grupos solúveis.

Mostramos no capítulo 3, que um dígrafo $D$ conexo, localmente finito e transitivo tem crescimento exponencial se pelo menos um dos expoentes $\exp ^{+}(D)$ ou $\exp ^{-}(D)$ é infinito. Conse- 
quentemente, estes expoentes são finitos se $D$ é um dígrafo conexo, localmente finito, transitivo e não tem crescimento exponencial. Então surge a seguinte pergunta:

Pergunta Será que é possivel encontrar um limite para $\exp ^{+}(D)$ e $\exp ^{-}(D)$ que dependa somente da taxa de crescimento de D ou de certas propriedades de grupos agindo transitivamente sobre $D$ ?

Mostraremos nos próximos resultados que isto é realmente possível. Primeiramente, consideraremos o caso em que um grupo $G$ age transitivamente sobre um dígrafo $D$ tal que $G$ possui um subgrupo normal abeliano $H$ agindo com um número finito de órbitas sobre $D$, desta forma obteremos uma cota superior para os expoentes, que dependa somente do número de órbitas de $H$ sobre $D$. Depois, exploraremos alguns resultados mais gerais, agora supondo que $H$ é nilpotente de classe $r$, chegaremos à uma cota superior para os expoentes dependendo somente do número de órbitas de $H$ sobre $D$ e de $r$. Inicialmente provamos alguns resultados auxiliares.

Lema 4.12. Seja $D$ um dígrafo conexo e $G$ um subgrupo transitivo de Aut $(D)$ que contém um subgrupo normal $H \unlhd G$ com $m$ órbitas sobre $D$, onde $1 \leq m<\infty$. Se para algum (e consequentemente todo) $u \in V D$ o conjunto $R_{1}^{+}(u)$ está contido em ${ }^{H} u$, então as seguintes afirmações são satisfeitas :

i) Para todo $v \in V D$ o conjunto $R^{+}(v)$ está contido em ${ }^{H} v$.

ii) O digrafo quociente $D_{H}$ é um ciclo direcionado.

Demonstração. Seja $v \in V D$. Se $m=1$, então $H$ age transitivamente sobre $D$, isto é, $V D={ }^{H} v$ e segue que $R^{+}(v) \subseteq{ }^{H} v$. Além disso, o dígrafo quociente $D_{H}$ tem somente um vértice, logo é um ciclo trivial. Assuma agora que $m \geq 2$.

Para provarmos (i), mostraremos que $R_{k}^{+}(v) \subseteq{ }^{H} v$, para todo $v \in V D$, usando indução sobre $k$. Por hipótese $R_{1}^{+}(v) \subseteq{ }^{H} v$, então o resultado vale para $k=1$. Agora, seja $k>1$ e suponha que $R_{j}^{+}(v) \subseteq{ }^{H} v$ vale para todo $j \leq k$. Sejam $w \in R_{k+1}^{+}(v)$ e

$$
W=\left(v_{0}, 1, v_{1}, \ldots, v_{n-1},-1, v_{n}\right)
$$

um passeio em $R_{k+1}[v, w]$. Observe que o indicador $\epsilon_{n}=-1$, caso contrário, teríamos que $F\left({ }_{0} W_{n-1}\right)=F(W)-\epsilon_{n}=-1$, absurdo. Suponha primeiro que para todo $0<i<n$ temos 
$F\left({ }_{0} W_{i}\right)>0$. Isto implica que $v_{1} R_{k}^{+} v_{n-1}$ pois

$$
F\left({ }_{1} W_{n-1}\right)=F(W)-\epsilon_{1}-\epsilon_{n}=0 .
$$

Além disso, para todo $0<j<n, F\left({ }_{1} W_{j}\right) \in[0, k]$. De fato, se para algum $j, F\left({ }_{1} W_{j}\right)<0$, temos que

$$
F\left({ }_{0} W_{j}\right)=F\left({ }_{1} W_{j}\right)+\epsilon_{1}<-1
$$

e segue que $F\left({ }_{0} W_{j}\right)=0$, pois ${ }_{0} W_{j} \in R_{k}^{+}[v, w]$, contradição. Agora, se $F\left({ }_{1} W_{j}\right)>k$, para algum $j$ temos

$$
F\left({ }_{0} W_{j}\right)=F\left({ }_{1} W_{j}\right)+1>k+1
$$

uma contradição. Portanto ${ }_{1} W_{j} \in R_{k}^{+}\left[v_{1}, v_{n-1}\right]$, como queríamos.

Por hipótese de indução temos que $R_{k}^{+}\left(v_{1}\right) \subseteq{ }^{H} v_{1}$, logo $v_{n-1} \in{ }^{H} v_{1}$. Então existe $h \in H$ tal que $v_{n-1}={ }^{h} v_{1}$. Assim, $\left({ }^{h} v_{0}, v_{n-1}\right)=\left({ }^{h} v_{0},{ }^{h} v_{1}\right)={ }^{h}\left(v_{0}, v_{1}\right) \in E D$, pois $\left(v_{0}, v_{1}\right) \in E D$. Dessa forma, existe o passeio $\left({ }^{h} v_{0}, 1, v_{n-1},-1, v_{n}\right)$ e portanto ${ }^{h} v_{0} \in R_{1}^{+}\left(v_{n}\right)$. Como por hipótese, $R_{1}^{+}\left(v_{n}\right) \subseteq{ }^{H} v_{n}$ segue que ${ }^{h} v_{0} \in{ }^{H} v_{n}$. Como $v=v_{0}$ e $v_{n}=w$, concluímos que $v \in{ }^{H} w$. Sejam $i_{1}, i_{2}, \ldots, i_{t}=n$ todos os índices satisfazendo $0<i_{1}<i_{2}<\ldots<i_{t}$ e $F\left({ }_{0} W_{i_{j}}\right)=0$. Considere o conjunto de subpasseios

$$
\mathcal{P}=\left\{{ }_{0} W_{i_{1}}, i_{1} W_{i_{2}}, \ldots, i_{t-1} W_{i_{t}}\right\}
$$

Note que para cada $W^{\prime} \in \mathcal{P}$ temos $F\left({ }_{0} W_{i}^{\prime}\right)>0$, ou seja, $W^{\prime}$ satisfaz as hipóteses de $W$ do caso anterior. Assim, obtemos que

$$
v_{i_{1}} \in{ }^{H} v ; v_{i_{2}} \in{ }^{H} v_{i_{1}} ; \ldots ; w \in{ }^{H} v_{i_{t-1}} .
$$

Logo $w \in{ }^{H} v$. E isto prova (i).

Agora provaremos (ii). Seja ${ }^{H} v$ uma $H$-órbita. Como $D$ é conexo e por hipótese $D$ tem pelo menos duas órbitas que são blocos de imprimitividade para $G$, existe uma $H$-órbita ${ }^{H} w \neq{ }^{H} v$ tal que $\left({ }^{H} w,{ }^{H} v\right) \in E D$. E portanto, pelo Lema 4.3 existe $w^{\prime} \in{ }^{H} w$ tal que $\left(w^{\prime}, v\right) \in E D$. Observe que o dígrafo quociente $D_{H}$ tem grau de entrada igual à 1 . De fato, caso contrário, existe uma $H$-órbita ${ }^{H} u$ tal que $\left({ }^{H} u,{ }^{H} v\right) \in E D$, onde ${ }^{H} u \neq{ }^{H} w$ e ${ }^{H} u \neq{ }^{H} v$. E do Lema 4.3, existe 
$u^{\prime} \in{ }^{H} u$ tal que $\left(u^{\prime}, v\right) \in E D$. Consequentemente, o passeio $W=\left(w^{\prime}, 1, v,-1, u^{\prime}\right)$ pertence à $R_{1}^{+}\left[w^{\prime}, u^{\prime}\right]$. E de (i), temos que $u^{\prime} \in{ }^{H} w$, uma contradição. Além disso, $D_{H}$ é finito, pois só temos um número finito de órbitas, e de $D$ conexo, segue que $D_{H}$ é um ciclo direcionado.

Lema 4.13. Seja $D$ um digrafo e seja $G$ um subgrupo transitivo de Aut $(D)$, tendo um subgrupo normal abeliano $H$ com $m$ órbitas sobre $D$, onde $1 \leq m<\infty$. Se para algum (e consequentemente todo) $u \in V D$ o conjunto $R_{1}^{+}(u)$ está contido em ${ }^{H} u$, então

$$
\exp ^{+}(D)=\exp ^{-}(D) \leq m
$$

Demonstração. Provaremos que $\exp ^{+}(D) \leq m$. Se $m=1$, então $H$ age transitivamente sobre $D$, e como $H$ é abeliano segue do Corolário 2.10 que $D$ é um dígrafo de Cayley de $H$. Logo, pela Proposição 4.8, $\exp ^{+}(D)=1$.

Assuma agora que $m \geq 2$. Seja $\Delta={ }^{H} u$, para algum $u \in V D$. Nosso primeiro passo é construir um dígrafo auxiliar $D^{*}$ com vértices $\Delta$ e aresta $(w, v)$ sempre que existir em $D$ um passeio direcionado de comprimento $m$ de $w$ à $v$. Observe que a restrição de $H$ a $\Delta$ age regularmente sobre $\Delta$, pois $H$ é abeliano. Consequentemente, $D^{*}$ é um dígrafo de Cayley de um grupo abeliano (possivelmente desconexo). Portanto, da Proposição 4.8 obtemos que $\exp ^{+}\left(D^{*}\right)=1$.

Agora, seja $v R^{+} w$, para algum $v, w \in V D$. Mostraremos que $v R_{m}^{+} w$. Por definição de $R^{+}$, temos que $v R_{k}^{+} w$ para algum inteiro $k \geq 1$. Se $k \leq m$, não há o que provar. Assuma que $k>m$. Pelo Lema 3.2, existe em $R_{k}^{+}[v, w]$ um passeio que é a concatenação de passeios da forma

$$
W=\left(v_{0}, 1, v_{1}, \ldots, 1, v_{k},-1, v_{k+1},-1, \ldots,-1, v_{2 k}\right)
$$

Pela transitividade da relação $R_{k}^{+}$, basta mostrar que $v_{0} R_{m}^{+} v_{2 k}$. Seja $t, r$ inteiros com $0 \leq r<m$ e tais que $k=t m+r$. Note que se $v_{1} \in{ }^{H} v_{0}$ então $\left({ }^{H} v_{0},{ }^{H} v_{1}\right)$ seria um laço, uma contradição pois, pelo Lema $4.12, D_{H}$ é um ciclo direcionado de comprimento $m$. Analogamente, se $v_{j} \in{ }^{H} v_{i}$ para algum $j>i, 0 \leq i<j \leq m-1$, então teríamos em $D_{H}$ um ciclo de comprimento $l<m$. Uma contradição, pois pelo Lema 4.12, $D_{H}$ é um ciclo direcionado de comprimento $m$. Logo, ${ }^{H} v_{0},{ }^{H} v_{1}, \ldots,{ }^{H} v_{m-1}$ são as distintas $m$ órbitas de $H$. Similarmente, po- 
demos mostrar que $v_{m}, v_{2 m}, \ldots, v_{t m}$ pertencem à ${ }^{H} v_{0}$. Além disso, para $j \in\{0,1, \ldots, t\}$, temos $v_{2 k-j m} \in R^{+}\left(v_{j m}\right)$ e pelo Lema $4.12 R^{+}\left(v_{j m}\right) \subseteq{ }^{H} v_{j m}$, concluímos que $v_{2 k}, v_{2 k-m}, \ldots, v_{2 k-t m}$ também pertencem à ${ }^{H} v_{0}$. Dessa forma, $v_{2 k}={ }^{h_{0}} v_{0}, v_{t m}={ }^{h_{1}} v_{0}$ e $v_{2 k-t m}={ }^{h_{2}} v_{0}$ para algum $h, h_{1}, h_{2} \in H$.

Agora note que $v_{t m}={ }^{h_{1}} v_{0}=h_{1} h_{2}^{-1} v_{2 k-t m}, \operatorname{logo}$

$$
W^{\prime}={ }_{0} W_{t m} \cdot\left({ }^{h_{1} h_{2}^{-1}}\left(2 k-t m W_{2 k}\right)\right)
$$

é um passeio de $v_{0}$ até $x=h_{1} h_{2}^{-1} v_{2 k}=h_{1} h_{2}^{-1} h_{v_{0}}$. Como ${ }_{0} W_{t m}$ é um passeio direcionado de comprimento tm e $\left(2 k-t m W_{2 k}\right)$ é um passeio direcionado de comprimento $t m$ e indicadores iguais à -1 , segue que $W^{\prime}$ é um passeio de $R_{t m}^{+}\left[v_{0}, x\right]$. De $H$ abeliano temos que $x={ }^{h h_{2}^{-1} h_{1}} v_{0}$, logo $h h_{2}^{-1}\left({ }_{t m} W_{2 k-t m}\right)$ é um passeio de $x$ à $v_{2 k}$. E de ${ }_{t m} W_{2 k-t m} \in R_{r}^{+}\left[v_{t m}, v_{2 k}\right]$ implica que $h h_{2}^{-1}\left({ }_{t m} W_{2 k-t m}\right) \in R_{r}^{+}\left[x, v_{2 k}\right]$. E da suposição de $r<m$, obtemos que $x R_{m}^{+} v_{2 k}$. Assim, $v_{0} R_{m}^{+} v_{2 k}$ se, e somente se, $v_{0} R_{m}^{+} x$.

De $v_{0} \in{ }^{H} v$ temos $v_{0}={ }^{h_{0}} v$ para algum $h_{0} \in H$. Suponha que $\Delta={ }^{H} v$, e então temos que $W^{\prime}$ corresponde à um passeio $W^{*} \in R_{t}\left[h_{0}, h_{1} h_{2}^{-1} h h_{0}\right]$ em $D^{*}$ pois, por construção, uma aresta de $D^{*}$ corresponde à um passeio direcionado de comprimento $m$ em $D$. Como, $\exp ^{+}\left(D^{*}\right)=1$, $W^{*}$ pode ser substituído por um passeio de $R_{1}^{+}\left[h_{0}, h_{1} h_{2}^{-1} h h_{0}\right]$, consequentemente $W^{\prime}$ pode ser substituído por um passeio de $R_{m}^{+}\left[v_{0}, x\right]$, $\operatorname{logo} x R_{m}^{+} v_{0}$, como queríamos. Portanto, $v R_{m}^{+} w$.

Deste modo, $R^{+} \subseteq R_{m}^{+}$implica que $\exp ^{+}(D) \leq m$. Analogamente, obtemos que $\exp ^{-}(D) \leq m$, daí pelo Corolário 3.10 completamos a prova.

Teorema 4.14. Seja $D$ um dígrafo. Seja $G \leq A u t(D)$ um subgrupo transitivo tendo um subgrupo normal abeliano $H$ agindo com $m, 1 \leq m<\infty$, órbitas sobre o conjunto de vértices de D. Então $\exp ^{+}(D)=\exp ^{-}(D) \leq m$

Demonstração. Provaremos por indução sobre $m$ que $\exp ^{+}(D) \leq m$. Se $m=1$ então $H$ age transitivamente sobre $D$, logo pela Proposição $4.8, \exp ^{+}(D)=1$. Agora, assuma que $m \geq 2$ e suponha que a afirmação é válida para todo $n<m$ e que $H$ age com $m$ órbitas sobre $D$. Se para algum $u \in V D$, temos $R_{1}^{+}(u) \subseteq{ }^{H} u$, então o resultado segue pelo Lema 4.13.

Assuma agora que as classes de equivalências com respeito à $R_{1}^{+}$não estão contidas nas $H$ órbitas e considere o dígrafo quociente $D / R_{1}^{+}$. Seja $K$ o núcleo da ação de $G$ sobre $D / R_{1}^{+}$e seja 
$N=\frac{H K}{K} \cong \frac{H}{H \cap K}$ a ação fiel induzida por $H$ em $D / R_{1}^{+}$. Observe que, como as $R_{1}^{+}$-classes de equivalência não estão totalmente contidas nas $H$-órbitas então cada $R_{1}^{+}$classe intercepta pelo menos duas órbitas, logo $N$ age com no máximo $\frac{m}{2}$ órbitas sobre $D / R_{1}^{+}$. Assim temos um grupo abeliano normal $N$ agindo com $\frac{m}{2}<m$ órbitas, então por hipótese de indução, $\exp ^{+}\left(D / R_{1}^{+}\right) \leq \frac{m}{2}$. E pela Proposição 4.1, obtemos

$$
\exp ^{+}(D)=\exp ^{+}\left(D / R_{1}^{+}\right)+1 \leq \frac{m+2}{2} \leq m
$$

pois $m \geq 2$. Analogamente, podemos mostrar que $\exp ^{-}(D) \leq m$. Portanto, $\exp ^{+}(D)$ e $\exp ^{-}(D)$ são ambos finitos, logo pelo Corolário 3.10, obtemos $\exp ^{+}(D)=\exp ^{-}(D) \leq m$.

Finalmente, podemos provar o resultado principal deste capítulo.

Teorema 4.15. Seja $G$ um grupo agindo transitivamente sobre um dígrafo conexo $D$ e seja $N \unlhd G$ um subgrupo nilpotente de classe $r$ agindo com $m$ órbitas sobre $D$, onde $1 \leq m<\infty$. Então $\exp ^{+}(D)=\exp ^{-}(D) \leq m(r+1)-1$.

Demonstração. Seja $G$ um grupo agindo transitivamente sobre um dígrafo conexo $D$ e seja $N \unlhd G$ nilpotente de classe $r$ agindo com $m$ órbitas sobre $D$, onde $1 \leq m<\infty$. Inicialmente provaremos que $\exp ^{+}(D) \leq m(r+1)-1$. A prova será feita por indução sobre $m$. Se $m=1$, então $N$ age transitivamente sobre $D$ e como $N$ é nilpotente, segue pelo Teorema 4.9 que $\exp ^{+}(D) \leq r$. Suponha agora que $m \geq 2$. Dividiremos em dois casos, dependendo da estrutura do dígrafo quociente $D_{N}$.

Caso 1 : O dígrafo quociente $D_{N}$ não é isomorfo ao ciclo direcionado com $m$ vértices, onde $m \geq 2$.

Neste caso, segue pelo Lema 4.12 que para qualquer $v \in V D$, o conjunto $R_{1}^{+}(v)$ não está totalmente contido em uma única órbita de $N$. Seja $\tau$ o sistema de imprimitividade de $G$ sobre $D$ que consiste das classes de equivalência com respeito à $R_{1}^{+}$. Então o grupo de permutação $G_{\tau}$, induzido pela ação de $G$ sobre $\tau$ age transitivamente sobre $D_{\tau}$. Além disso, $N_{\tau}$ age com no máximo $\frac{m}{2}$ órbitas sobre $D$. Note também que $N_{\tau}$ é nilpotente de classe no máximo $r$, pois $N_{\tau}$ é uma imagem homomórfica de $N$. Daí, por hipótese de indução obtemos que $\exp ^{+}\left(D_{\tau}\right) \leq \frac{m}{2}(r+1)-1$ 
e pela Proposição 4.1 obtemos

$$
\exp ^{+}(D)=\exp ^{+}\left(D_{\tau}\right)+1 \leq \frac{m}{2}(r+1)-1+1
$$

Agora, como $m \geq 2$, temos $\frac{m}{2}(r+1) \geq 1$, e segue que

$$
\exp ^{+}(D) \leq \frac{m}{2}(r+1) \leq m(r+1)-1
$$

Portanto, $\exp ^{+}(D) \leq m(r+1)-1$.

Caso $2 D_{N}$ é isomorfo ao ciclo direcionado $C=\left(c_{1}, c_{2}, \ldots, c_{m}\right)$ com $m \geq 2$ vértices.

Seja $O_{1}, O_{2}, \ldots, O_{m}$ as distintas $m$ órbitas de $N$ sobre o conjunto $V D$ correspondentes aos vértices $c_{1}, c_{2}, \ldots, c_{m}$. Note que num ciclo direcionado não há laços, logo não existe aresta em $D$ que liga dois vértices que estão na mesma órbita. Além disso, todas as arestas em $D$ são direcionadas de $O_{i}$ para $O_{i+1}, 1 \leq i \leq m$, onde os índices são tomados módulo $m$.

Afirmação 1: Para $1 \leq i \leq m$ e $v \in O_{i}$ temos $R^{+}(v) \subseteq O_{i}$

Pela transitividade de $G$ em $D$ é suficiente provar a afirmação para $i=1$. Seja $w \in R^{+}(v)$. Por definição existe $k \geq 1$ tal que $v R_{k}^{+} w$, assim existe um passeio em $R_{k}^{+}[v, w]$ que é formado por concatenações de passeios

$$
W=\left(u_{0}, 1, u_{1}, 1, \ldots, 1, u_{k},-1, \ldots,-1, u_{2 k}\right)
$$

Dessa forma basta provar que se $u_{0} \in O_{1}$ então $u_{2 k} \in O_{1}$. Como as arestas em $D_{N}$ estão direcionadas de $O_{i}$ para $O_{i+1}$ e não existe aresta em um mesmo $O_{i}$ então de $u_{0}$ a $u_{k}$ percorremos $k$ órbitas, e voltamos pelas mesma $k$ órbitas ao percorremos de $u_{k+1}$ até $u_{k}$. Logo $u_{2 k} \in O_{1}$.

Observe que se $R_{m-1}^{+}(v)=O_{i}$, então da Afirmação 1 obtemos que $R^{+}(v) \subseteq O_{i}=R_{m-1}^{+}(v)$ $\operatorname{logo} \exp ^{+}(D) \leq m-1$. Com isso, temos que considerar somente o caso quando uma $R_{m-1}^{+}$-classe está propriamente contida em $O_{i}$, para todo $i, 1 \leq i \leq m$ e para todo vértice $v \in O_{i}$.

Denotaremos por

- $B_{\iota}, \iota \in \mathcal{I}$ : classes de equivalências de $R_{m-1}^{+}$em $O_{1}$.

- $\mathcal{P}^{m}$ : conjunto formado por todos os passeios direcionados $P=\left(v_{1}, \ldots, v_{m+1}\right)$ em $D$, onde 
$v_{j} \in O_{j}$ para $1 \leq j \leq m$ e $v_{m+1} \in O_{1}$.

- $\mathcal{P}^{-m}$ : conjunto de todos passeios inversos de $\mathcal{P}^{m}$.

- $\mathcal{R}_{m-1}^{+}$: conjunto de todos passeios que estão contidos em $R_{m-1}^{+}[u, v]$, para alguns vértices $u, v \in O_{1}$.

Sejam $v_{1}, v_{2} \in O_{1}$ vértices tais que $v_{1} R^{+} v_{2}$. Se $v_{1}$ e $v_{2}$ estão ambos contidos no mesmo conjunto $B_{\iota}, \iota \in \mathcal{I}$, então $v_{1} R_{m-1}^{+} v_{2}$, e como $(m-1)<m$ o resultado segue. Agora, suponha que $v_{1} \in B_{\iota_{1}}$ e $v_{2} \in B_{\iota_{2}}$, com $\iota_{1} \neq \iota_{2}$. Seja $D^{\prime}$ o dígrafo que tem como conjunto de vértices o conjunto $\mathcal{I}$ e aresta $\left(\iota_{1}, \iota_{2}\right)$ sempre que existir um passeio $P \in \mathcal{P}^{m}$ com vértice inicial em $B_{\iota_{1}}$ e vértice final em $B_{\iota_{2}}$. Observe que em geral o dígrafo $D^{\prime}$ não é localmente finito, pois podemos ter infinitos tais caminhos. No entanto, do fato de $N$ ser transitivo em $O_{1}$ então temos que $N$ induz um grupo transitivo agindo sobre $D^{\prime}$ que é nilpotente de classe no máximo $r$. Assim, o Teorema 4.9 implica que $\exp ^{+}\left(D^{\prime}\right) \leq r$.

Por definição, temos $v_{1} R_{k}^{+} v_{2}$ para algum $k \geq 1$ e segue do Lema 3.2 que existe um passeio em $R_{k}^{+}\left[v_{1}, v_{2}\right]$ formado por concatenações de passeios da forma

$$
W=\left(u_{0}, 1, \ldots, u_{k},-1, \ldots,-1, u_{2 k}\right)
$$

Pela transitividade de $R^{+}$basta mostrarmos que $u_{0} R_{m(r+1)-1}^{+} u_{2 k}$. Assim, escrevendo $k=m t+r^{\prime}$, onde $t, r^{\prime} \in \mathbb{Z}^{+}$e $r^{\prime}<m$, fica claro que $W$ é um passeio formado pela concatenação de $t$ caminhos de $\mathcal{P}^{m}$, seguido por um passeio de $\mathcal{R}_{m-1}^{+}$e então $t$ passeios de $\mathcal{P}^{-m}$.

Sejam $u_{0}, u_{1}, \ldots, u_{2 t+1}$ os vértices de $W$, contido em $O_{1}$, obtidos nessa ordem ao percorremos $W$. Assim, $u_{0}, u_{1}, \ldots, u_{t-1}$ são as origens dos caminhos de $\mathcal{P}^{m}$, enquanto o vértice $u_{t}$ é o vértice inicial do passeio de $\mathcal{R}_{m-1}^{+}$e os vértices $u_{t+1}, u_{t+2}, \ldots, u_{2 t}$ são as origens dos caminhos $\mathcal{P}^{-m}$. Logo $W$ é definido por

$$
W=(u_{0}, \underbrace{1, \ldots, 1}_{m}, u_{1}, \overbrace{1, \ldots, 1}^{m}, u_{2}, 1, \ldots, 1, u_{t}) \cdot W^{*} \cdot(u_{t+1}, \underbrace{-1, \ldots,-1}_{m}, u_{t+2},-1, \ldots,-1, u_{2 t+1})
$$

onde o passeio $W^{*} \in R_{m-1}^{+}\left[u_{t}, u_{t+1}\right], u_{0}=v_{1}, u_{2 t+1}=v_{2}$, deste modo temos que $W \in$ 
$R_{m t+m-1}^{+}\left[v_{1}, v_{2}\right]$. Consequentemente $W$ dá origem a um passeio $W^{\prime}$ em $D^{\prime}$, dado por

$$
W^{\prime}=\left(\iota_{0}^{\prime}, 1, \iota_{1}, 1, \iota_{2}^{\prime}, 1, \ldots, 1, \iota_{t-1}^{\prime}, 1, \iota_{t+1}^{\prime},-1, \iota_{t+2}^{\prime},-1, \ldots,-1, \iota_{2 t+1}^{\prime}\right)
$$

onde para cada $i$ temos que $u_{i} \in B_{\iota_{i}^{\prime}}$ (observe que $u_{t} \in B_{\iota_{t+1}^{\prime}}=B_{\iota_{t}^{\prime}}$, pois $u_{t} R_{m-1}^{+} u_{t+1}$ ). Note que $W^{\prime} \in R_{t}^{+}\left[\iota_{0}^{\prime}, \iota_{2 t+1}^{\prime}\right]$ logo, como $\exp ^{+}\left(D^{\prime}\right) \leq r$ então podemos substituir $W^{\prime}$ por um passeio de $R_{r}^{+}\left[\iota_{0}^{\prime}, \iota_{2}^{\prime}\right]$ e consequentemente podemos substituir $W$ por um passeio de $R_{m(r+1)-1}^{+}\left[u_{0}, u_{2 k}\right]$. Portanto, então $\exp ^{+}(D) \leq m(r+1)-1$.

De forma análoga obtemos que $\exp ^{-}(D) \leq m(r+1)-1$. E o Corolário 3.10 implica que $\exp ^{+}(D)=\exp ^{-}(D) \leq m(r+1)-1$.

Corolário 4.16. Seja $G$ um grupo finitamente gerado, seja $N$ um subgrupo normal nilpotente de indice finito $m$ em $G$ e seja $D$ o dígrafo de Cayley de $G$ com respeito a algum conjunto finito de geradores $S$. Então $\exp ^{+}(D)=\exp ^{-}(D) \leq m(r+1)-1$ vale, onde $r$ é a classe de nilpotência de $N$.

É natural perguntar se esta cota é a melhor possível já que todos exemplos conhecidos, de fato satisfazem $\exp ^{+}(D)=\exp ^{-}(D) \leq m r$. Assim, temos o seguinte problema.

Problema 4.1. É verdade que $\exp ^{+}(D)=\exp ^{-}(D) \leq m r$ vale para todo dígrafo de Cayley dos grupos descritos no Corolário 4.16?

\subsection{Observações finais}

Concluiremos este trabalho com as seguintes observações. Seja $G$ um subgrupo de $\operatorname{Aut}(D)$ agindo transitivamente sobre um dígrafo $D$ com expoentes $\exp ^{+}(D)$ e $\exp ^{-}(D)$ ambos finitos. Conforme observado após a demonstração do Lema 4.7: as classes de equivalências da relação $R^{+}=R^{-}$são órbitas de um subgrupo normal de $G$. Portanto, se esta relação não é universal e se o dígrafo tem grau de entrada ou grau de saída pelo menos 2 (de forma que as classes tenham pelo menos dois elementos), então este subgrupo normal de $G$ é próprio e não-trivial. Consequentemente, se $G$ é simples, a relação $R^{+}=R^{-}$é universal em $D$. Conforme mostrado no Teorema 3.21, um dígrafo transitivo, infinito, conexo e localmente finito $D$ tem crescimento exponencial se pelo menos um dos expoentes $\exp ^{+}(D)$ ou $\exp ^{-}(D)$ é infinito. Neste momento 
relembramos o seguinte problema da teoria combinatória de grupos (veja [2]), originalmente proposto por R. I. Grigorchuk.

Problema 4.2. Todo grupo simples, infinito e finitamente gerado tem crescimento exponencial?

A seguinte proposição nos permite então formular uma conjectura que relaciona intimamente este problema com relações de alcance.

Proposição 4.17. Se um grupo simples infinito finitamente gerado $G$ não possui crescimento exponencial, então para todo conjunto finito $S$ de geradores de $G$ existe um inteiro finito $k_{s} \geq 1$, tal que $R_{k_{s}}^{+}=R_{k_{s}}^{-}$é universal em $\operatorname{Cay}(G, S)$.

Demonstração. Seja $S$ um conjunto finito de geradores de $G$ e seja $D=\operatorname{Cay}(G, S)$. Então $D$ é um dígrafo conexo, infinito e localmente finito. Como $G$ não tem crescimento exponencial, segue do Teorema 3.21, que os expoente $\exp ^{+}(D)$ e $\exp ^{-}(D)$ são ambos finitos. Pelo Lema 4.7, $\exp ^{+}(D)=\exp ^{-}(D)$, segue que existe $k_{s} \geq 1$ tal que $R_{k_{s}}^{+}=R_{k_{s}}^{-}$. Pela simplicidade de $G$, temos pelo Lema 4.7 que $R_{k_{s}}^{+}=R_{k_{s}}^{-}$é universal.

Conjectura 4.1. Seja $G$ um grupo simples, infinito e finitamente gerado. Então existe um conjunto finito de geradores $S$ de $G$ tal que para o dígrafo de Cayley $D$ de $G$ com relação a $S$ uma das seguintes condiçôes é satisfeita:

(a) Pelo menos um dos expoentes $\exp ^{+}(D)$ ou $\exp ^{-}(D)$ é infinito e portanto $D$ tem crescimento exponencial.

(b) Ambos os expoentes $\exp ^{+}(D)$ e $\exp ^{-}(D)$ são finitos e as relações de alcance $R^{+}$e $R^{-}$não são universal sobre $D$.

Suponha que a conjectura seja válida e seja $G$ um grupo simples infinito finitamente gerado. Pela conjectura, existe $S \subset G$ um conjunto finito de geradores tais que $D=C a y(G, S)$ satisfaz (a) ou (b). Se (a) é satisfeita então $D$ tem crescimento exponencial (e portanto, $G$ tem crescimento exponencial). Agora suponha que (b) seja satisfeita e suponha, por contradição que $D$ não tenha crescimento exponencial. Então, pela Proposição 4.17, existe $k \geq 1$ tal que $R_{k}^{+}=R_{k}^{-}$ é universal (porque vale para todo $\operatorname{Cay}(G, S)$ então vale para todo $D$ ). Portanto $R^{+}=R^{-}$é 
universal (porque se $R_{k}^{+}$nos dá todo $V D$ e $R_{k}^{+} \subset R^{+}$, então é claro que $R^{+}$será universal). Uma contradição. Segue que $D$ tem crescimento exponencial.

Portanto, se a Conjectura 4.1 é válida então obtemos uma resposta positiva para o Problema 4.2 de Grigorchuk . 


\section{Referências Bibliográficas}

[1] D. Amato. Descendants in infinite, primitive, highly arc-transitive digraphs, Discrete Mathematics 310, 2021-2036.

[2] G. Baumslag, A. G. Myasnikov e V. Shpilrain, Open problems in combinatorial group theory, $2^{\text {a }}$ edição, Contemporary Mathematics, 296, AMS (2002).

[3] L. Bartholdi e W. Woess, Spectral computations on Lamplighter groups and Diestel-Leader graphs, Journal of Fourier analysis and applications, 11, Springer, (2005), 175-202.

[4] M. Bhattacharjee, D. Macpherson, R. G. Möller, P. M. Neumann, Notes on Infinite Permutation Groups, Springer (1698).

[5] P. J. Cameron, C. E. Praeger, N. C. Wormald, Infinite Highly arc-transitive digraphs and universal covering digraphs, Combinatorica 13 (1993) 377-396.

[6] M. Devos, B. Mohar, R. Šámal, Highly arc-transitive digraphs - structure and counterexamples, Combinatorica 35 (2012), 553-571.

[7] J. D. Dixon e B. Mortimer,Permutation groups, Springer Science (1996)

[8] M. Gromov, Groups of polynomial growth and expanding maps, Inst. Hautes Etudes Sci. Publ. Math. 53 (1981), 53-78.

[9] I. M. Isaacs, Algebra: a graduate course, American Mathematical Society (2009).

[10] A. Malnič, D. Marušič, R. Möller, N. Seifter, V. I. Trofimov, B. Zgrablič, Highly arctransitive digraphs: Reachability, topological groups, European J. Combin. 26 (2005) 19-28. 
[11] A. Malnič, D. Marušič, N. Seifter, P. Šparl e B. Zgrabilič, Reachability relations in digraphs, Europ. J. Combin. 29 (2008), $1566-1581$.

[12] A. Malnič, D. Marušič, B. Zgrablič, Highly arc-transitive digraphs with no homomorphism onto Z, Combinatorica 22 (2002), 435-443.

[13] A. Malnič, P. Potočnik, N. Seifter, P. Šparl, Reachability relations, transitive digraphs and groups, ARS Mathemathica Contemporanea 8 (2015), 83-94.

[14] A. Mann, How groups grow, LMS Lecture Notes Ser. 395, Cambridge Uni. Press, 2012.

[15] A. Malnič, P. Potočnik, Bridging semisymmetric and half-arc-transitive actions on graphs, European J. Combin. 23 (2002) 719-732.

[16] C. E. Praeger, On homomorphic images of edge transitive directed graphs, Australas. J. Combin. 3 (1991) 207-210.

[17] J. J. Rotman, An introduction to the theory of groups, Springer-Verlag (1994).

[18] N. Seifter e V. I. Trofimov, Reachability relations and the structure of transitive digraphs, Electron. J. Combin. 16 (2009), R26.

[19] V. I. Trofimov, On geometric properties of directed vertex-symmetric graphs, European J. Combin. 27 (2006), $690-700$.

[20] W. Woess, Lamplighters, Diestel-Leader graphs, random walks, and harmonic functions, Combinatorics, Probability and Computing. 14, (2005), 415-433. 